\title{
AN X-RAY SEARCH FOR COMPACT CENTRAL SOURCES IN SUPERNOVA REMNANTS. II. SIX LARGE-DIAMETER SNRs
}

\author{
D. L. Kaplan, ${ }^{1}$ B. M. Gaensler, ${ }^{2,3}$ S. R. Kulkarni, ${ }^{4}$ and P. O. Slane ${ }^{2}$ \\ Received 2005 November 18; accepted 2006 January 13
}

\begin{abstract}
We present the second in a series of studies in which we have searched for undiscovered neutron stars in supernova remnants (SNRs). This paper deals with the six largest SNRs in our sample, too large for Chandra or XMM-Newton to cover in a single pointing. These SNRs are nearby, with typical distances of $<1 \mathrm{kpc}$. We therefore used the ROSAT Bright Source Catalog and past observations in the literature to identify X-ray point sources in and near the SNRs. Out of 54 sources, we were immediately able to identify optical/IR counterparts to 41 from existing data. We obtained Chandra snapshot images of the remaining 13 sources. Of these, 10 were point sources with readily identified counterparts, two were extended, and one was not detected in the Chandra observation but is likely a flare star. One of the extended sources may be a pulsar wind nebula, but if so it is probably not associated with the nearby SNR. We are then left with no identified neutron stars in these six SNRs down to luminosity limits of $\sim 10^{32} \mathrm{ergs} \mathrm{s}^{-1}$. These limits are generally less than the luminosities of typical neutron stars of the same ages, but are compatible with some lower luminosity sources such as the neutron stars in the SNRs CTA 1 and IC 443.
\end{abstract}

Subject headings: pulsars: general — stars: neutron — supernova remnants $-\mathrm{X}$-rays: stars

Online material: color figures

\section{INTRODUCTION}

The connection between core-collapse supernovae and neutron stars (Baade \& Zwicky 1934) has had a solid observational footing for almost 40 years, due largely to the discovery of young radio pulsars in supernova remnants (SNRs) such as Vela (Large et al. 1968) and in the Crab Nebula (Staelin \& Reifenstein 1968). Energetic young pulsars like these are strong radio and X-ray sources and often power synchrotron nebulae called pulsar wind nebulae ( PWNe; Gaensler \& Slane 2006) that are indirect markers of pulsars (e.g., Camilo 2003).

The idea that young neutron stars resemble the Crab pulsar came to dominate the search for the products of supernovae (e.g., Kaspi et al. 1996). Recently, however, young neutron stars have been revealed in a wide variety of manifestations, from anomalous X-ray pulsars (AXPs) and soft $\gamma$-ray repeaters, to nearby thermal and radio-quiet neutron stars, to long-period radio pulsars with high inferred magnetic fields. As exemplified by the identification of the central compact object in the Cas A SNR (Tananbaum 1999), much of this diversity has come from X-ray observations.

While this diversity is clearly demonstrated observationally, theory and simulation cannot yet constrain the fundamental birth properties of neutron stars (e.g., Burrows et al. 2004; Chevalier 2005). Models still have difficulties achieving explosions, much less following the activity in the postcollapse object in any detail.

Kaplan et al. (2004, hereafter Paper I) have attempted to address our lack of understanding of stellar death and neutron star cooling by defining a volume-limited $(d<5 \mathrm{kpc})$ sample of SNRs, examining the neutron stars that they contain, and outlining a sur-

\footnotetext{
1 Pappalardo Fellow; Kavli Institute for Astrophysics and Space Research, Massachusetts Institute of Technology, 77 Massachusetts Avenue, Room 37664H, Cambridge, MA 02139; dlk@space.mit.edu.

2 Harvard-Smithsonian Center for Astrophysics, 60 Garden Street, MS 6, Cambridge, MA 02138; bgaensler@cfa.harvard.edu, slane@cfa.harvard.edu.

3 Alfred P. Sloan Research Fellow.

4 Department of Astronomy, MS 105-24, California Institute of Technology, Pasadena, CA 91125; srk@astro.caltech.edu.
}

vey designed to detect or significantly constrain neutron stars in the remaining remnants. The primary subsample discussed in Paper I is one where the SNR diameter is $<45^{\prime}$, so the Chandra $X$-Ray Observatory can observe a significant fraction of the SNR interior with its ACIS-I detector and hence cover the area where neutron stars would be within a reasonable range of velocities $\left(v_{\perp}<700 \mathrm{~km} \mathrm{~s}^{-1}\right.$, where $v_{\perp}$ is the velocity perpendicular to the line of sight). Paper I also discuss two other subsamples of SNRs: one with diameters $45^{\prime}<\theta<90^{\prime}$ for which $X M M$-Newton is suitable (and which we will present in a forthcoming paper), and one with $\theta>90^{\prime}$. It is this subsample of the six largest SNRs from Paper I that we consider here.

The organization of the paper is as follows. In $\S 2$ we give brief summaries of the six SNRs discussed here. In $\S 3$ we describe our identification of candidate X-ray sources in and around the SNRs. In $\S 4$ we detail the initial identification of optical/IR counterparts to the X-ray sources using available sky surveys; as discussed in Paper I, optical/IR observations are a powerful way to reject $\mathrm{X}$-ray sources that are not neutron stars (see also, e.g., Rutledge et al. 2003). With the sky surveys we were able to identify most of the X-ray sources with high confidence; those for which we were not certain were selected for additional Chandra observations and optical/IR observations ( $(5)$. Finally, we give our discussion and conclusions in $\S 6$. All coordinates are J2000.0.

\section{SUPERNOVA REMNANTS}

We list the SNRs for this paper, along with relevant parameters, in Table 1. Each SNR has a distance determined from a more reliable method than the $\Sigma$ - $D$ method (e.g., Huang \& Thaddeus 1985; Case \& Bhattacharya 1998), but they are not all of the same quality. In the best cases, the distances are from kinematic observations of optical or radio lines. In the worst cases, the distances are from fitting shock models to the X-ray data (e.g., Kassim et al. 1994). These distances involve many uncertainties beyond the kinematic distances, including the assumption of a Sedov-phase remnant, the state of equilibration in the system, the nonsphericity of the explosion, and the unknown total explosion energy. For 
TABLE 1

LARGE SNRs

\begin{tabular}{|c|c|c|c|c|c|c|}
\hline SNR & Other Name & $\begin{array}{c}\text { Size } \\
(\operatorname{arcmin})\end{array}$ & $\begin{array}{c}D \\
(\mathrm{kpc})\end{array}$ & Distance Method & $\begin{array}{c}N_{\mathrm{H}} / 10^{21} \mathrm{a} \\
\left(\mathrm{cm}^{-2}\right)\end{array}$ & $\begin{array}{l}L_{\mathrm{X}} / 10^{31 \mathrm{~b}} \\
\left(\mathrm{ergs} \mathrm{s}^{-1}\right)\end{array}$ \\
\hline G65.3+5.7 & $\mathrm{G} 65.2+5.7$ & $310 \times 240$ & 0.8 & Optical velocity & 1.4 & 6.7 \\
\hline 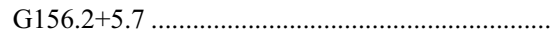 & & 110 & 1.3 & NEI fits & 3.5 & 29 \\
\hline G160.9+2.6 & HB 9 & $140 \times 120$ & 1.5 & H I, optical velocity & 1 & 21 \\
\hline G205.5+0.5 & Monoceros & 220 & 1.2 & Optical velocity & 0.8 & 13 \\
\hline G330.0+15.0 & Lupus Loop & 180 & 1.2 & NEI fits & 0.5 & 12.0 \\
\hline
\end{tabular}

NotE.-See $\S 2$ for a general discussion about the quality of the remnant distances and for detailed discussions about each remnant.

${ }^{a}$ Hydrogen column density to SNR. Derived from previous observations (if available), otherwise determined from measured H I absorption or using COLDEN integrated over velocity range appropriate for the SNR distance.

${ }^{\mathrm{b}}$ Unabsorbed X-ray luminosity $(0.3-8.0 \mathrm{keV})$ of a nominal $0.05 \mathrm{~s}^{-1}$ ROSAT PSPC source at the distance and absorption of the SNR, assuming a blackbody spectrum with $k T=0.25 \mathrm{keV}$ (this allows for easy conversion of count rates to luminosities, assuming that the sources are associated with the SNRs).

kinematic distances, the uncertainties are probably $\lesssim 30 \%$, but for distances from X-ray fitting they could exceed 50\% (Kassim et al. 1994). The ages tend to be derived from X-ray fits for all sources, although having an independently determined distance for some sources makes for better constraints. Below we discuss each SNR in more detail.

\subsection{SNR G65.3+5.7}

SNR G65.3+5.7 (also known as G65.2+5.7) was identified as an SNR by Gull et al. (1977) by its filamentary line emission. It has major axes of $310^{\prime} \times 240^{\prime}$. According to Mavromatakis et al. (2002), the age is $20-25 \mathrm{kyr}$ and the distance is $\approx 0.8-1.0 \mathrm{kpc}$ (a kinematic distance derived from the velocity of optical emission lines; also see Lozinskaya 1981). Mavromatakis et al. (2002) show data from pointed ROSAT (Röntgensatellit) observations (Schaudel et al. 2002) but do not discuss point sources; the ROSAT data detect emission from much of the interior at $>2.5 \times 10^{-4}$ counts $\mathrm{s}^{-1}$ pixel $^{-1}$, with $45^{\prime \prime}$ pixels. Shelton et al. (2004) do mention an extended ( $\gtrsim 6^{\prime}$ radius) soft source at $19^{\mathrm{h}} 36^{\mathrm{m}} 46^{\mathrm{s}},+30^{\circ} 40^{\prime} 07^{\prime \prime}$, but come to no conclusion as to its identity. The inner $3^{\prime}$ were searched for radio pulsars by Gorham et al. (1996) at $1410 \mathrm{MHz}$ down to a limit of $0.1 \mathrm{mJy}$, but given the size of SNR G65.3+5.7 a transverse velocity of only $v_{\perp}=20 \mathrm{~km} \mathrm{~s}^{-1}$ would have moved a neutron star outside the search region, so the lack of detection was not very constraining.

The $0.58 \mathrm{~s}$ radio pulsar PSR J1931+30 lies $45^{\prime}$ from the center of the remnant. However, with no estimate of the spin-down rate (and hence no spin-down age), the period seems rather large to be associated with an SNR (it would require an unusual but not unheard of magnetic field of $\sim 10^{13} \mathrm{G}$ ), and no definite claim of an association can be made (Schaudel et al. 2002).

\subsection{SNR G74.0-8.5}

SNR G74.0-8.5, also known as the Cygnus Loop, is a $230^{\prime} \times$ $160^{\prime}$ radio and X-ray shell. The distance, estimated from measurements of the shock velocity and proper motion, is $0.44 \mathrm{kpc}$ (Blair et al. 1999), and the age is $8 \mathrm{kyr}$ (Levenson et al. 2002). Miyata et al. (1998a) reported $\approx 8.8$ counts $\mathrm{s}^{-1}$ in the interior over the $22^{\prime}$ field of the SIS for their ASCA (Advanced Satellite for Cosmology and Astrophysics) observation, and used these data to conclude that SNR G74.0-8.5 was likely the result of a Type II supernova on the basis of elemental abundances.

Miyata et al. (1998b) searched SNR G74.0-8.5 for promising $\mathrm{X}$-ray point sources that might be compact objects and identified two, one of which they later concluded was an active galactic nucleus on the basis of its long-term variability, X-ray spectrum, and radio counterpart (Miyata et al. 2001), and the other of which they conclude may be a neutron star. The inner $10^{\prime}$ were searched for radio pulsars by Gorham et al. (1996) down to a $430 \mathrm{MHz}$ flux limit of $0.3 \mathrm{mJy}$, but given the size of SNR G74.0-8.5 the lack of detection was not very constraining $\left(v_{\perp} \leq 80 \mathrm{~km} \mathrm{~s}^{-1}\right)$. The inner $30^{\prime}$ were also searched by Biggs $\&$ Lyne (1996) for pulsars down to a $400 \mathrm{MHz}$ flux of $3 \mathrm{mJy}$. Assuming an average radio spectrum for radio pulsars of $S_{\nu} \propto \nu^{-1.5}$, this translates to a $1400 \mathrm{MHz}$ luminosity limit of $0.01 \mathrm{mJy} \mathrm{kpc}^{2}$, which is considerably fainter than the very low luminosity PSR J0205+6449 (0.5 mJy kpc ${ }^{2}$; Camilo et al. 2002).

\subsection{SNR G156.2+5.7}

SNR G156.2+5.7 was discovered in the ROSAT All-Sky Survey by Pfeffermann et al. (1991). It has a faint $110^{\prime}$ shell in both $\mathrm{X}$-rays and radio, and nonequilibrium fits to the X-ray data place it at a distance of $\approx 1.3 \mathrm{kpc}$ with an age of $15 \mathrm{kyr}$ (Yamauchi et al. 1999). Lorimer et al. (1998) searched SNR G74.0-8.5 for radio pulsars, tiling seven pointings of the $76 \mathrm{~m}$ Lovell telescope at Jodrell Bank, each of which covered $\approx 0.5$. The search did not find any pulsars, down to a flux limit of $0.7 \mathrm{mJy}$ at $606 \mathrm{MHz}$, or a $1400 \mathrm{MHz}$ luminosity limit of $0.3 \mathrm{mJy} \mathrm{kpc}^{2}$.

\subsection{SNR G160.9+2.6}

SNR G160.9+2.6, also known as HB 9, is a $140^{\prime} \times 120^{\prime}$ radio shell with bright X-rays in the interior. Leahy \& Aschenbach (1995) use X-ray fitting to estimate a distance of $1.5 \mathrm{kpc}$ and an age of 8-20 kyr. This distance is consistent with the upper limit of $4 \mathrm{kpc}$ derived from other measurements (Lozinskaya 1981; Leahy \& Roger 1991).

The inner $30^{\prime}$ were also searched by Biggs \& Lyne (1996) for pulsars down to a $610 \mathrm{MHz}$ flux of $15 \mathrm{mJy}$. Damashek et al. (1978) discovered an old radio pulsar (PSR B0458+46) in the interior of the SNR, although the association between the pulsar and the SNR is generally considered to be false (e.g., Kaspi \& Helfand 2002) based on the large spin-down age and low spindown energy-loss rate for the pulsar $\left(10^{6} \mathrm{yr}\right.$ and $10^{33} \mathrm{ergs} \mathrm{s}^{-1}$, respectively).

\subsection{SNR G205.5+0.5}

SNR G205.5+0.5, also known as the Monoceros nebula, is a $220^{\prime}$ radio shell. The systemic velocity of optical line emission puts the SNR at a distance of $0.8 \mathrm{kpc}$ (Lozinskaya 1981), although distances up to $1.6 \mathrm{kpc}$ are preferred by low-frequency radio data that show the SNR within the Mon OB2 association 
(Odegard 1986). The age is likely $\sim 30 \mathrm{kyr}$, as inferred from fits to X-ray data (Leahy et al. 1986).

\subsection{SNR G330.0+15.0}

SNR G330.0+15.0, the Lupus Loop, is a low surface brightness radio shell approximately $180^{\prime}$ in diameter. Nonequilibrium fits to the X-ray data and comparison with the column density of the nearby remnant of SN 1006 suggest a distance of $1.0-1.2 \mathrm{kpc}$ and an age of 50 kyr (Leahy et al. 1991).

\section{SOURCE SELECTION}

Chandra or XMM-Newton imaging of the entire fields of the large-diameter SNRs listed in Table 1 is impractical because of their sizes. Their proximities $(d \lesssim 1 \mathrm{kpc})$, however, mean that we do not need the high sensitivities of Chandra or XMM-Newton to achieve the same luminosity limit as in Paper I. We therefore used the ROSAT All-Sky Survey Bright Source Catalog (BSC; Voges et al. 1999) for our source selection. This was a survey of the entire sky with the Position Sensitive Proportional Counter (PSPC) aboard ROSAT.

The positional accuracy of the PSPC does not approach that of Chandra or even XMM-Newton (typical uncertainties are $10^{\prime \prime}$ ), and the observations are not as deep as the pointed Chandra and $X M M-N e w t o n$ observations used for the other SNRs. Nonetheless, the BSC is useful. As seen in Table 1, its limit of 0.05 counts s$^{-1}$ in the PSPC is actually of roughly comparable depth to our Chandra observations in Paper I, $(1-10) \times 10^{31} \mathrm{ergs} \mathrm{s}^{-1}$, when the smaller distances and column densities of the SNRs in this paper are taken into account. While the X-ray positions do not in all cases allow unambiguous optical identifications, the relative brightness and softness of the X-ray sources compared to those in Paper I mean that very often stars from the Digitized Sky Survey (DSS) or Two Micron All Sky Survey (2MASS; Skrutskie et al. 1997) can be identified as counterparts.

We selected the BSC sources within twice the nominal radii (for elliptical sources, we took the semimajor axes) of the SNRs in Table 1 (as defined by their positions and sizes given by Green 2000) that had $\geq 0.05$ counts $\mathrm{s}^{-1}$ and were listed as unextended (a value of 0 in the ext column of the BSC catalog). Searching outside the remnants allowed us to find neutron stars that have overtaken the SNR shocks - not an uncommon occurrence (van der Swaluw et al. 2004) in SNRs of the ages considered here (10-30 kyr). This gave us all of the X-ray sources listed in Table 2. For the sake of comparison between sources, we plot the distribution of hardness ratios in Figure 1.

\subsection{Extended Sources}

In our analysis, we rejected those BSC sources that were identified as extended. This was for several reasons: we eliminated peaks in diffuse background emission that may have been identified as discrete sources, and we eliminated large extended objects such as galaxy clusters. Practically, point sources offer much better astrometry and are better suited to counterpart identification.

However, in some sense our selection was less than ideal. We would have eliminated any bright PWNe, although these might have been identified by previous searches. Also, source confusion makes our resulting luminosity limits less constraining than they might otherwise be, as two nearby point sources could have been identified as a single extended source and hence been rejected. Given the relatively low space density of BSC sources (Fig. 2) this should not be a major effect, but it should still be noted. In contrast, our Chandra observations do not suffer from any confusion limitations.
One might ask if the diffuse emission from the SNRs themselves will limit the depth of the BSC in the SNR interiors. We have found in general that this is not the case. Figure 2 shows the density of BSC sources (both pointlike and of all sizes) within different radii from the SNR centers. While the inner reaches of the SNRs have few sources and therefore poor statistics, in no case is there a statistically significant deficit of point sources inside the SNR. There might be a slight deficit inside SNR G156.2+5.7 or SNR G160.9+2.6, but these are also the smallest of the SNRs and therefore have the fewest total sources. Similarly, in Figure 3 we show the average background count rates determined when extracting the sources plotted in Figure 2, with the same binning. Two of the SNRs (G65.3+5.7 and G74.0-8.5) do show background increases in the interiors, two do not (G205.5+0.5 and G330.0+15.0), and two are uncertain due to few counts (G156.2+5.7 and G160.9+2.6), but even an increase of a factor of 3 above the mean background rate $\left(\approx 10^{-3}\right.$ counts $\mathrm{s}^{-1}$ arcmin $^{-2}$ ) would give only $\approx 0.005$ counts s ${ }^{-1}$ within the $90 \%$ confidence radius of a PSPC source (for $0.3 \mathrm{keV}$ ), ${ }^{5}$ which is a factor of 10 less than the minimum source count rate for the BSC. Therefore, the diffuse SNR emission should not have significantly affected the BSC source detection, and it is unlikely that there were any point sources that were missed.

\subsection{Additional Sources}

Besides the BSC, we took advantage of X-ray observations in the literature to identify additional sources for Chandra followup. These were as follows: for SNR G74.0-8.5, AX J2049.6+ 2939 (from ASCA; Miyata et al. 1998b, 2001); and for SNR G205.5+0.5, Einstein sources 1, 3, and 6 from Leahy et al. (1986), known as 1E 0627.4+0537, 1E 0630.9+0611, and 1E 0636.8+ 0517 , respectively.

\section{COUNTERPART IDENTIFICATION}

Once we had assembled the list of X-ray sources, we then examined the publicly available surveys (DSS, $2 \mathrm{MASS},{ }^{6}$ and the NRAO VLA Sky Survey ${ }^{7}$ [NVSS; Condon et al. 1998]), as well as examination of SIMBAD and the relevant literature. With these sources of information, we were able to identify likely counterparts to 41 of the 50 sources in Table 2 . We list the relevant data (X-ray and optical) of the identifications in Table 2, with a summary of all identifications and additional notes in Table 3. The separations between the nominal X-ray and optical positions were consistent with the predicted X-ray position uncertainties (Fig. 4).

There are a number of cases where there were multiple stars within the X-ray error circles, some of which were known to be physically associated with each other (as noted in SIMBAD). In these cases we list multiple possible counterparts in Table 2. The true source of the X-ray emission may be any one of the stars, or may in fact come from the interactions between them.

While the identifications were made only on the basis of positional coincidence with bright stars, in many cases we can be additionally confident. This is because the stars are so bright $(V<5 \mathrm{mag})$ that the chances of a false association are negligible or the stars are of types known to have X-ray emission (e.g., $\mathrm{T}$ Tauri stars). To aid in the evaluation of our identifications, we

\footnotetext{
5 See http://heasarc.gsfc.nasa.gov/docs/heasarc/caldb/docs/rosat/cal_ros_92_ 001/cal_ros_92_001.html.

6 When we were doing the initial source selection, the final 2MASS data had not been released, so there were cases where we made decisions based only on DSS data.

For all SNRs but SNR G330.0+15.0, which is below the $\delta=-40^{\circ}$ limit of the NVSS.
} 
TABLE 2

ROSAT Point Sources and Stellar Counterparts

\begin{tabular}{|c|c|c|c|c|c|c|c|c|c|c|c|}
\hline Number & 1RXS J & $\begin{array}{c}\text { PSPC } \\
\left(\text { counts } \mathrm{s}^{-1} \text { ) }\right.\end{array}$ & $\begin{array}{c}\Delta R^{\mathrm{b}} \\
(\operatorname{arcmin})\end{array}$ & $\begin{array}{c}\sigma r^{\mathrm{c}} \\
(\operatorname{arcsec})\end{array}$ & $\begin{array}{c}\text { R.A. }^{\mathrm{a}} \\
(\mathrm{J} 2000.0)\end{array}$ & $\begin{array}{c}\text { Decl. }^{\mathrm{a}} \\
(\mathrm{J} 2000.0)\end{array}$ & Star & 2MASS J & $\begin{array}{c}\delta r^{\mathrm{d}} \\
(\operatorname{arcsec})\end{array}$ & $\begin{array}{c}V \\
(\mathrm{mag})\end{array}$ & $\begin{array}{c}K_{s} \\
(\mathrm{mag})\end{array}$ \\
\hline \multicolumn{12}{|c|}{ SNR G65.3+5.7: } \\
\hline $1 \ldots \ldots$. & $193445.6+303100$ & 0.066 & 45.1 & 11 & 193445.23 & +303058.9 & HD 184738 & $19344524+3030590$ & 5.0 & 10.41 & 8.108 \\
\hline $2 \ldots \ldots \ldots$ & $193840.0+303035$ & 0.083 & 82.7 & 9 & 193840.10 & +303028.0 & V* EM Cyg & $19384012+3030284$ & 7.1 & 12.6 & 11.150 \\
\hline $3 \ldots \ldots \ldots$ & $193922.4+300921$ & 0.054 & 101.8 & 12 & 193922.61 & +300912.0 & HD 185734 & $19392261+3009119$ & 9.9 & 4.685 & 2.499 \\
\hline \multirow[t]{2}{*}{$4 \ldots \ldots \ldots$} & $194337.2+322523$ & 0.256 & 155.8 & 8 & 194336.80 & +322520.7 & BDS 9566B & $19433674+3225206$ & 5.6 & 9.9 & 8.082 \\
\hline & & & & & 194337.90 & +322512.7 & HD 331149 & $19433790+3225124$ & 13.6 & 10.7 & 7.179 \\
\hline $5 \ldots \ldots \ldots$ & $193458.1+335301$ & 0.051 & 165.0 & 14 & 193458.10 & +335301.5 & $\ldots$ & $\ldots$ & $\ldots$ & $\ldots$ & $\ldots$ \\
\hline $6 \ldots \ldots \ldots$ & $192722.3+280934$ & 0.110 & 194.4 & 15 & 192721.91 & +280942.8 & USNO 1181-0406270 & $19272197+2809452$ & 9.8 & $11.620^{\mathrm{e}}$ & 8.426 \\
\hline $7 \ldots \ldots \ldots$ & $194401.5+284456$ & 0.138 & 202.7 & 8 & 194401.37 & +284509.9 & GSC 02151-03394 & $19440138+2845099$ & 14.0 & $\ldots$ & 8.691 \\
\hline $8 \ldots \ldots \ldots$ & $194902.9+295258$ & 0.357 & 219.9 & 7 & 194902.99 & +295258.3 & HD 187460 & $19490298+2952582$ & 1.2 & 8.32 & 5.734 \\
\hline $9 \ldots \ldots \ldots$ & $193228.6+345318$ & 0.091 & 223.4 & 9 & 193228.59 & +345318.5 & $\ldots$ & $\ldots$ & $\ldots$ & $\ldots$ & $\ldots$ \\
\hline $10 \ldots \ldots$ & $191449.0+315131$ & 0.057 & 237.0 & 14 & 191450.21 & +315137.3 & HD 180314 & $19145022+3151371$ & 16.5 & 6.618 & 4.312 \\
\hline $11 \ldots \ldots$ & $193856.2+351407$ & 0.290 & 255.7 & 8 & 193855.77 & +35 1413.0 & HD 185696 & $19385576+3514132$ & 8.0 & 8.29 & 6.858 \\
\hline $12 \ldots \ldots$ & $193936.8+263718$ & 0.074 & 285.6 & 8 & 193936.67 & +263716.1 & $\mathrm{AG}+262090$ & $19393666+2637169$ & 3.0 & 11.1 & 7.345 \\
\hline $13 \ldots \ldots$ & $193113.0+360730$ & 0.153 & 298.4 & 7 & 193112.57 & +360730.0 & G125-15 & $19311257+3607300$ & 13.1 & $\ldots$ & 8.839 \\
\hline $14 \ldots \ldots$ & $191151.1+285012$ & 0.052 & 305.3 & 11 & 191150.81 & +285007.6 & USNO 1188-0330651 & $19115080+2850075$ & 5.7 & $12.510^{\mathrm{e}}$ & 8.898 \\
\hline \multicolumn{12}{|c|}{ SNR G74.0-8.5: } \\
\hline $1 \ldots \ldots \ldots$ & $205042.9+284643$ & 0.111 & 113.3 & 12 & 205042.90 & +284643.5 & $\ldots$ & $\ldots$ & $\ldots$ & $\ldots$ & $\ldots$ \\
\hline $2 \ldots \ldots \ldots$ & $204457.4+291613$ & 0.104 & 114.4 & 10 & 204458.09 & +291621.3 & HD 335070 & $20445809+2916211$ & 11.9 & 10.8 & 8.746 \\
\hline \multirow[t]{2}{*}{$3 \ldots \ldots \ldots$} & $205812.8+292037$ & 0.129 & 122.3 & 8 & 205812.82 & +292028.6 & USNO 1193-0519643 & $20581282+2920282$ & 8.9 & $15.980^{\mathrm{e}}$ & 14.258 \\
\hline & & & & & 205812.80 & +292037.5 & & $20581257+2920454$ & 8.5 & & 13.405 \\
\hline $4 \ldots \ldots \ldots$ & $205208.5+270546$ & 0.225 & 214.7 & 8 & 205207.68 & +270549.1 & HD 198809 & $20520768+2705491$ & 11.4 & 4.576 & 2.722 \\
\hline \multicolumn{12}{|c|}{ SNR G156.2+5.7: } \\
\hline $1 \ldots \ldots$. & $050639.1+513607$ & 0.051 & 75.3 & 20 & 050640.63 & +513551.8 & HD 32537 & $05064067+5135519$ & 20.8 & 4.980 & 4.124 \\
\hline \multicolumn{12}{|c|}{ SNR G160.9+2.6: } \\
\hline $1 \ldots \ldots \ldots$ & $045707.4+452751$ & .061 & 82.5 & 9 & 045707.40 & +452751.0 & $\cdots$ & $\cdots$ & $\cdots$ & $\ldots$ & $\cdots$ \\
\hline $2 \ldots \ldots \ldots$ & $050339.8+451715$ & 0.061 & 87.2 & 11 & 050339.80 & +451715.0 & $\ldots$ & $\ldots$ & $\ldots$ & $\ldots$ & $\ldots$ \\
\hline $3 \ldots \ldots \ldots$ & $045222.2+455619$ & 0.052 & 99.0 & 10 & 045221.51 & +455623.7 & HD 30736 & $04522151+4556236$ & 8.6 & 6.695 & 5.407 \\
\hline \multicolumn{12}{|c|}{ SNR G205.5+0.5: } \\
\hline \multirow{2}{*}{\multicolumn{2}{|c|}{$1 \ldots \ldots . \quad 064108.3+052250$}} & 0.086 & 74.3 & 11 & 064108.07 & +052252.1 & USNO 0155-01104-1 & $06410807+0522522$ & 4.0 & $10.780^{\mathrm{e}}$ & 8.905 \\
\hline & & & & & 064107.96 & +052243.8 & & $06410796+0522438$ & 8.0 & $\ldots$ & 12.121 \\
\hline $2 \ldots \ldots \ldots$ & $064136.2+080218$ & 0.054 & 100.1 & 11 & 064135.94 & +080205.6 & HD 262113 & $06413601+0802055$ & 13.0 & 10.3 & 8.712 \\
\hline \multirow[t]{4}{*}{$3 \ldots \ldots \ldots$} & $064109.3+044733$ & 0.076 & 107.4 & 17 & 064109.61 & +044735.8 & USNO 0947-0100759 & $06410953+0447354$ & 5.4 & $17.650^{\mathrm{e}}$ & 15.084 \\
\hline & & & & & 064109.82 & +044735.1 & USNO 0947-0100763 & $06410988+0447350$ & 8.1 & $15.840^{\mathrm{e}}$ & 14.636 \\
\hline & & & & & 064109.00 & +044723.9 & $\ldots$ & $06410900+0447239$ & 10.1 & $\ldots$ & 12.182 \\
\hline & & & & & 064109.27 & +044718.7 & USNO 0155-02167-1 & $06410924+0447187$ & 14.3 & $11.100^{\mathrm{e}}$ & 8.688 \\
\hline $4 \ldots \ldots \ldots$ & $064641.1+082152$ & 0.092 & 160.1 & 10 & 064640.73 & +082147.3 & HD 49015 & $06464073+0821471$ & 7.6 & 7.5 & 6.080 \\
\hline $5 \ldots \ldots \ldots$ & $062740.3+073103$ & 0.082 & 179.5 & 8 & 062740.30 & +073103.0 & & $\ldots$ & $\ldots$ & $\ldots$ & $\ldots$ \\
\hline $6 \ldots \ldots \ldots$ & $062937.2+082930$ & 0.088 & 183.9 & 9 & 062936.89 & +082932.8 & HD 45759 & $06293689+0829327$ & 5.1 & 7.62 & 6.306 \\
\hline \multirow[t]{3}{*}{$7 \ldots \ldots \ldots$} & $063715.7+032005$ & 0.109 & 191.7 & 10 & 063715.22 & +032008.0 & USNO 0150-00332-1 & $06371522+0320081$ & 7.6 & $10.640^{\mathrm{e}}$ & 9.323 \\
\hline & & & & & 063715.85 & +032004.3 & $\ldots$ & $06371585+0320043$ & 2.5 & $\ldots$ & 14.508 \\
\hline & & & & & 063715.55 & +032002.6 & $\ldots$ & $06371555+0320026$ & 3.7 & $\ldots$ & 11.813 \\
\hline $8 \ldots \ldots \ldots$ & $062554.8+065543$ & 0.106 & 196.7 & 11 & 062555.24 & +065538.8 & USNO 0145-01717-1 & $06255524+0655386$ & 7.8 & $9.660^{\mathrm{e}}$ & 8.019 \\
\hline \multicolumn{12}{|c|}{ SNR G330.0+15.0: } \\
\hline $1 \ldots \ldots \ldots$ & $150818.8-401730$ & 0.077 & 26.1 & 10 & 150818.80 & -401730.0 & $\cdots$ & $\ldots$ & $\ldots$ & $\cdots$ & $\ldots$ \\
\hline \multirow[t]{3}{*}{$2 \ldots \ldots \ldots$} & $151059.6-392655$ & 0.082 & 35.0 & 12 & 151059.06 & -392658.5 & USNO 0505-0350285 & $15105908-3926590$ & 7.2 & $\ldots$ & 15.066 \\
\hline & & & & & 151058.25 & -392650.2 & USNO 7826-00179-1 & $15105821-3926499$ & 16.4 & $10.600^{\mathrm{e}}$ & 8.374 \\
\hline & & & & & 151059.72 & -392656.9 & USNO 0505-0350290 & & 2.4 & $\ldots$ & \\
\hline $3 \ldots \ldots \ldots$ & $150814.0-403445$ & 0.069 & 40.3 & 16 & 150812.12 & -403502.1 & HD 133880 & $15081213-4035022$ & 27.1 & 5.762 & 5.934 \\
\hline $4 \ldots \ldots \ldots$ & $150428.9-392423$ & 0.051 & 72.7 & 11 & 150428.65 & -392426.1 & CD -38 9913 & $15042865-3924261$ & 3.9 & 10.7 & 8.293 \\
\hline $5 \ldots \ldots \ldots$ & $150526.4-385709$ & 0.073 & 81.8 & 8 & 150526.01 & -385700.8 & RX J1505.4-3857 & $15052586-3857031$ & 9.4 & 12.55 & 9.124 \\
\hline $6 \ldots \ldots \ldots$ & $150139.6-403815$ & 0.125 & 103.2 & 14 & 150139.60 & -403815.5 & $\ldots$ & $\ldots$ & $\ldots$ & $\ldots$ & $\ldots$ \\
\hline $7 \ldots \ldots \ldots$ & $151849.8-405108$ & 0.107 & 113.6 & 17 & 151852.82 & -405052.8 & V* LX Lup & $15185282-4050528$ & 38.4 & 11.01 & 8.547 \\
\hline $8 \ldots \ldots \ldots$ & $145951.7-401158$ & 0.085 & 117.1 & 11 & 145952.44 & -401159.5 & HD 132349 & $14595244-4011594$ & 8.6 & 9.90 & 8.401 \\
\hline $9 \ldots \ldots \ldots$ & $151659.2-382648$ & 0.065 & 123.0 & 16 & 151659.35 & -382651.4 & HD 135549 & $15165935-3826514$ & 3.4 & 6.876 & 5.789 \\
\hline $10 \ldots \ldots$ & $152046.2-405405$ & 0.074 & 135.1 & 10 & 152046.97 & -405352.7 & HD 136206 & $15204697-4053526$ & 15.1 & 7.83 & 6.518 \\
\hline $11 \ldots \ldots$ & $145837.5-391507$ & 0.084 & 138.2 & 10 & 145837.56 & $\begin{array}{lll}-39 & 15 & 02.7\end{array}$ & USNO 0507-0344267 & $14583744-3915033$ & 4.9 & $11.850^{\mathrm{e}}$ & 8.648 \\
\hline $12 \ldots \ldots$ & $152211.8-395958$ & 0.080 & 140.1 & 11 & 152211.75 & -395949.6 & $\mathrm{~V}^{*} \mathrm{LZ}$ Lup & $15221162-3959509$ & 8.4 & 12.02 & 9.100 \\
\hline $13 \ldots \ldots$ & $145721.8-401401$ & 0.099 & 145.9 & 9 & 145722.07 & -401358.6 & $\ldots$ & $14572207-4013586$ & 4.2 & $\ldots$ & 13.333 \\
\hline $14 \ldots \ldots$ & $151806.7-380423$ & 0.115 & 148.5 & 12 & 151807.15 & -380423.8 & $\ldots$ & $15180715-3804238$ & 5.3 & $\ldots$ & 10.600 \\
\hline & & & & & 151807.61 & -380423.6 & USNO 7822-00433-1 & $15180762-3804237$ & 10.7 & $10.650^{\mathrm{e}}$ & 7.999 \\
\hline $15 \ldots \ldots$ & $151446.3-422020$ & 0.054 & 150.7 & 13 & 151447.48 & -422014.9 & RX J1514.8-4220 & $15144748-4220149$ & 14.2 & $\ldots$ & 9.011 \\
\hline
\end{tabular}


TABLE 2-Continued

\begin{tabular}{|c|c|c|c|c|c|c|c|c|c|c|c|}
\hline Number & 1RXS J & $\begin{array}{c}\text { PSPC } \\
\left(\text { counts s }{ }^{-1}\right)\end{array}$ & $\begin{array}{c}\Delta R^{\mathrm{b}} \\
(\operatorname{arcmin})\end{array}$ & $\begin{array}{c}\sigma r^{\mathrm{c}} \\
(\operatorname{arcsec})\end{array}$ & $\begin{array}{c}\text { R.A. }^{\mathrm{a}} \\
(\mathrm{J} 2000.0)\end{array}$ & $\begin{array}{c}\text { Decl. }^{\mathrm{a}} \\
(\mathrm{J} 2000.0)\end{array}$ & Star & 2MASS J & $\begin{array}{c}\delta r^{\mathrm{d}} \\
(\operatorname{arcsec})\end{array}$ & $\begin{array}{c}V \\
(\mathrm{mag})\end{array}$ & $\begin{array}{c}K_{s} \\
(\mathrm{mag})\end{array}$ \\
\hline $16 \ldots \ldots$ & $152012.2-382159$ & 0.153 & 152.8 & 8 & 152012.53 & -382157.9 & $C D-3710147 C$ & $15201253-3821579$ & 4.2 & & 8.454 \\
\hline $18 \ldots \ldots$ & $152445.4-394238$ & 0.105 & 170.4 & 11 & 152445.01 & -394237.0 & HD 136933 & $15244501-3942367$ & 4.7 & 5.367 & 5.495 \\
\hline $19 \ldots \ldots$ & $145613.6-385121$ & 0.176 & 172.5 & 8 & 145614.04 & -385120.1 & HD 131675 & $14561404-3851200$ & 5.2 & 9.15 & 7.323 \\
\hline $20 \ldots \ldots$ & $145744.3-414140$ & 0.051 & 173.7 & 15 & 145744.90 & -414138.8 & USNO 0483-0366208 & $14574495-4141394$ & 6.9 & $11.790^{\mathrm{e}}$ & 9.351 \\
\hline
\end{tabular}

Notes.-Stellar identifications were made only on the basis of the ROSAT data and SIMBAD. See also Table 3 and $\S 4$. Units of right ascension are hours, minutes, and seconds, and units of declination are degrees, arcminutes, and arcseconds.

a This is the position of the optical counterpart if known; otherwise, it is the X-ray position.

${ }^{\mathrm{b}}$ Separation between the X-ray source and the nominal SNR center given by Green (2000).

c X-ray position uncertainty.

${ }^{\mathrm{d}}$ Separation between the X-ray and optical sources.

${ }^{\text {e }}$ No $V$ magnitude was available from SIMBAD, so this is the $R 2$ magnitude from USNO-B1.0.

plot the cumulative number density of 2MASS sources for each SNR in Figure 5. For a typical position uncertainty of $10^{\prime \prime}$, we expect that all identifications with 2MASS sources brighter than $K_{s} \approx 11 \mathrm{mag}$ will be real (chance probability $<1 \%$ ), and even for sources brighter than $K_{s} \approx 13 \mathrm{mag}$ the identifications will be probable (chance rates $<10 \%$ ). We note, however, that while 1RXS J205812.8+292037 is consistent with having an association with one of the identified 2MASS sources, the association is not secure. A Chandra follow-up observation likely would have been definitive, as it was for the majority of the ambiguous sources discussed in $\S 5$, but due to an oversight on our part this source was not selected for follow-up Chandra observations. We therefore discuss 1RXS J205812.8+292037 in extra detail in $\S 5.3 .3$.

Given the uncertainties in spectrum and foreground column density, virtually all of the X-ray sources are consistent with being stars (as opposed to active galaxies; Fig. 6). We show 2MASS images of the X-ray sources with optical counterparts indicated in Figures $7-18$.

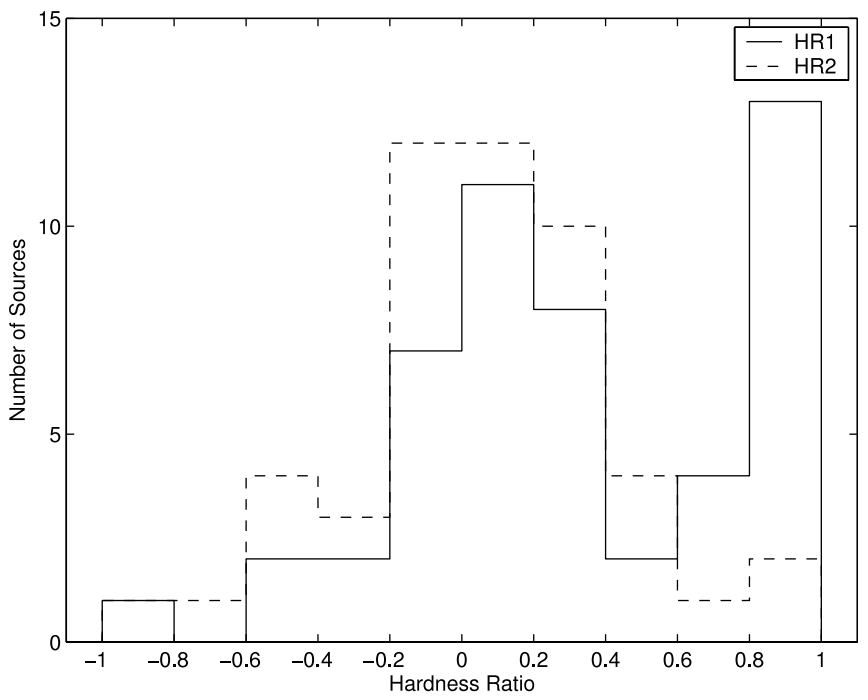

Fig. 1.-Hardness ratios of BSC sources from Table 2. The solid line shows HR1, while the dashed line shows HR2. HR $1 \equiv(B-A) /(B+A)$ and HR2 $\equiv$ $(D-C) /(D+C)$, where $A, B, C$, and $D$ are the count rates in the PHA ranges $11-41,52-201,52-90$, and $90-201$, respectively, and the PHA values correspond roughly to the energies in eV (Voges et al. 1999). As discussed in Voges et al. (1999), we note that HR2 is constructed only from counts in the $B$ range, so it is not a contradiction to have (for example) HR1 $=-1$ and HR2 $=1$.

\section{CHANDRA OBSERVATIONS}

The nine BSC sources that had no obvious optical counterparts (excluding 1RXS J205812.8+292037, as mentioned above), plus the four sources from $\S 3.2$, were selected for Chandra followup observations. Here, as in Paper I, we selected the exposure times (3-6 ks) based on the known column densities to the SNRs (Table 1) and a blackbody spectrum with $k T=0.25 \mathrm{keV}$. The positions are known to sufficient accuracy to allow use of the ACIS-S3 CCD (Garmire et al. 2003). Depending on the source brightnesses, however, we were concerned about photon pileup for some of the sources, so we used the 1/2- or 1/4-subarray modes (which also provides improved timing information), depending on the positional uncertainties. A log of the observations is in Table 4.

In most of the cases, the Chandra observations revealed nothing extraordinary. In the case of the BSC sources, the Chandra data typically showed that the BSC position was significantly off and/or the counterpart was faint (Figs. 19 and 20). The additional four sources from the literature were all coincident with stellar sources, once we had Chandra positions. Of the 13 sources with Chandra follow-up, nine had pointlike Chandra sources with obvious IR counterparts ( $(5.1)$. Of the other four sources, two show extended X-ray emission with Chandra, one has no obvious 2MASS counterpart, and one source was not detected in the Chandra observation. We discuss these sources in more detail in $\S$ 5.3.

\subsection{Notes on Chandra Sources}

In the cases where Chandra point sources were detected, the counterpart identifications are essentially secure. This is due to the very small positional uncertainty of the Chandra positions $\left(<1^{\prime \prime}\right)$ coupled with the brightnesses of the counterparts (see Fig. 5 and Paper I). Figures 19 and 20 contain images of those sources with counterpart identifications. With these identifications we can eliminate these sources as candidate compact objects using the X-ray-to-optical flux ratio (see Fig. 6 and Paper I). Here we comment on all of the sources observed with Chandra.

$1 R X S J 193458.1+335301$. - The Chandra source is extended. See $\S$ 5.3.1.

IRXS J193228.6+345318. - The Chandra source is pointlike and is at $19^{\mathrm{h}} 32^{\mathrm{m}} 27^{\mathrm{s}} .25,+34^{\circ} 53^{\prime} 14^{\prime \prime} .8\left(17^{\prime \prime}\right.$ away from the BSC position). It is coincident with the $K_{s}=14.2 \mathrm{mag}$ source 2MASS J19322722+3453148, the $B=16.8 \mathrm{mag}$ source USNO 1248-0333432, and with NVSS source identified in Table 3. It was identified as a flare star by Fuhrmeister \& Schmitt (2003) after our initial source selection. 


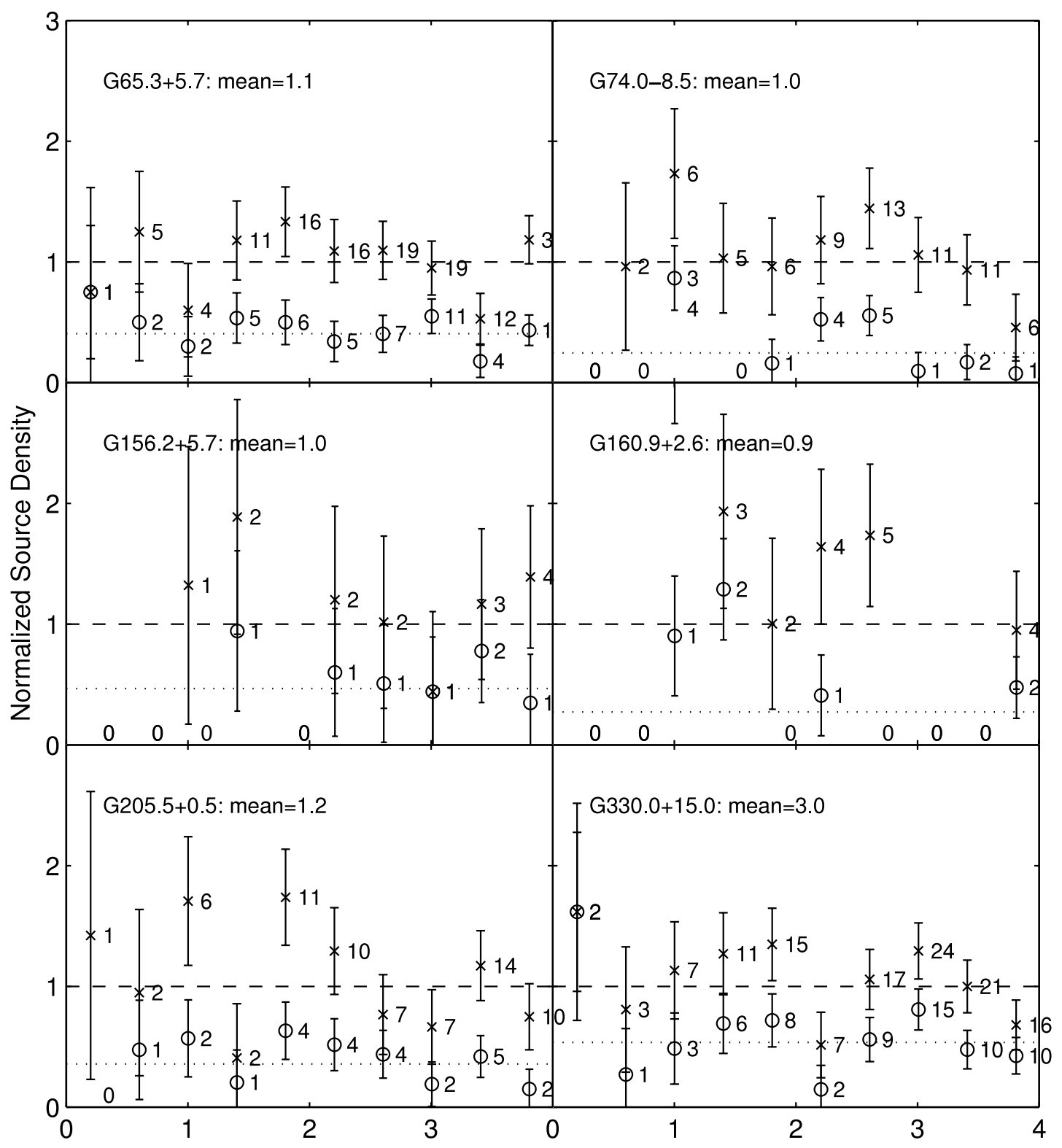

Radius / SNR Radius

FIG. 2.- Normalized density of BSC sources in each of the SNRs from Table 1. The number of sources per square arcminute divided by the mean density is plotted

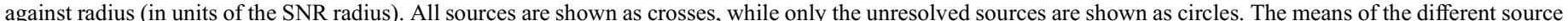

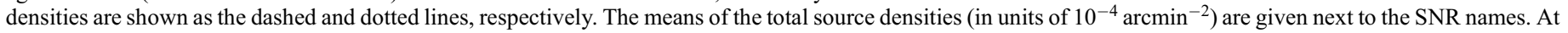

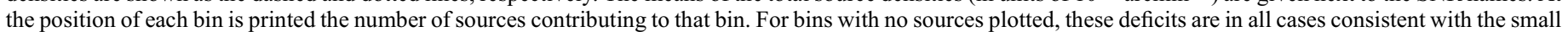

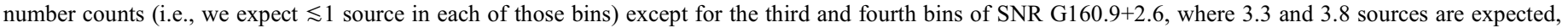
respectively. However, even in these bins there is no significant deficit of point sources.

$1 R X S$ J205042.9+284643.- - There is no Chandra source in the follow-up observation. See $\S$ 5.3.2.

$A X J 2049.6+2939$. - This $A S C A$ source was identified as a possible neutron star by Miyata et al. (1998b, 2001). The Chandra source is pointlike and is at $20^{\mathrm{h}} 49^{\mathrm{m}} 35^{\mathrm{s}} .41,+29^{\circ} 38^{\prime} 50^{\prime \prime} .9$. It is coincident with the $K_{s}=10.0$ mag source 2MASS J20493540+ 2938509 (also USNO 1196-0518650), which is presumably the $V=12.6$ mag G star discussed by Miyata et al. (1998b). Our observed count rate for this source $\left(\approx 0.03 \mathrm{~s}^{-1}\right.$ in the $0.3-2.0 \mathrm{keV}$ band) is roughly comparable with that predicted from the latest $A S C A$ spectroscopy, although as noted in Miyata et al. (2001), the source appears to be variable. Given the variability of this source and the extremely tight coincidence with a $\mathrm{G}$ star [in this region, there are $(1.97 \pm 0.07) \times 10^{-5} \operatorname{arcsec}^{-2}$ stars with $K \leq 10.0 \mathrm{mag}$ in 2MASS, and finding one $<0$ "' 2 away from the X-ray source has a chance rate of $\approx 2 \times 10^{-6}$; see Fig. 5], the $X$-ray emission is very likely from an active star. Miyata et al. (1998b) had dismissed this possibility because of the high X-ray intensity, but it is in fact consistent with the majority of the stars that we detect here (Fig. 6; the X-ray flux is $\sim 10^{-13} \mathrm{ergs} \mathrm{s}^{-1} \mathrm{~cm}^{-2}$ ).

IRXS J045707.4+452751. - The Chandra source is pointlike and is at $04^{\mathrm{h}} 57^{\mathrm{m}} 08.31,+45^{\circ} 27^{\prime} 49^{\prime \prime} .8\left(10^{\prime \prime}\right.$ away from the BSC position). It is coincident with the $K_{s}=14.5 \mathrm{mag}$ source $2 \mathrm{MASS}$ J04570832+4527499. 


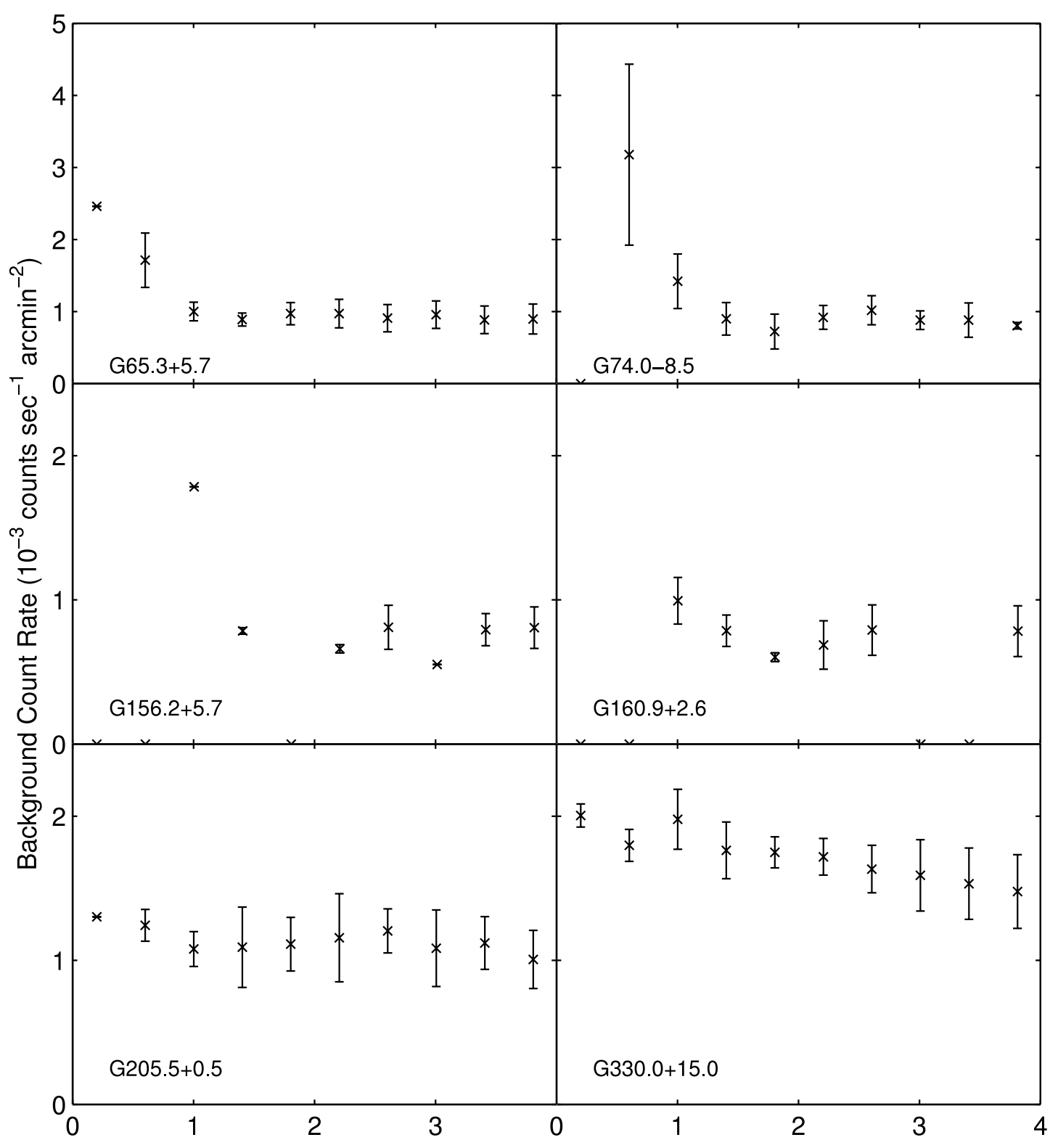

Radius / SNR Radius

FIG. 3.-Background count rate vs. radius for the BSC sources in each of the SNRs from Table 1. The average background count rate $\left(10^{-3} \mathrm{counts} \mathrm{s}^{-1}\right.$ arcmin $\left.^{-2}\right)$ in each of 10 radial bins between 0 and 4 times the SNR radius is shown, along with uncertainties showing the standard deviation in each bin. SNRs G65.3+5.7 and G74.0-8.5 do show a factor of 2-3 increase in background rate inside the SNRs. For SNRs G156.2+5.7 and G160.9+2.6, the situation is not as clear because there are very few sources inside (see Fig. 2). For SNRs G205.5+0.5 and G330.0+15.0, there do not appear to be significant rises toward the interiors.

1RXS J050339.8+451715.- The Chandra source is pointlike and is at $05^{\mathrm{h}} 03^{\mathrm{m}} 39^{\mathrm{s}} .59,+45^{\circ} 16^{\prime} 59^{\prime \prime} .5\left(15^{\prime \prime}\right.$ away from the BSC position). It is coincident with the $K_{s}=15.0$ mag source $2 \mathrm{MASS}$ J05033958+4516594 and with the NVSS source identified in Table 3. There is no USNO counterpart.

$1 R X S$ J062740.3+073103.- - The Chandra source is pointlike and is at $06^{\mathrm{h}} 27^{\mathrm{m}} 40^{\mathrm{s}} \cdot 12,+07^{\circ} 31^{\prime} 00^{\prime \prime} 3$ ( $4^{\prime \prime}$ from the BSC position). It is coincident with the $K_{s}=10.1 \mathrm{mag}$ source 2MASS J06274012+0731006.

$1 E$ 0627.4+0537. - The Chandra source is pointlike and is at $06^{\mathrm{h}} 30^{\mathrm{m}} 05^{\mathrm{s}} .29,+05^{\circ} 45^{\prime} 40^{\prime \prime} 8$. It is coincident with the $K_{s}=$ 10.0 mag source 2MASS J06300529+0545407.

$1 E$ 0630.9+0611. - The Chandra source is pointlike and is at $06^{\mathrm{h}} 33^{\mathrm{m}} 33.22,+06^{\circ} 08^{\prime} 39^{\prime \prime} .5$. It is coincident with the $K_{s}=$ 13.4 mag source 2MASS J06333322+0608396.
$1 E 0636.8+0517$. - The Chandra source is pointlike and is at $06^{\mathrm{h}} 39^{\mathrm{m}} 25^{\mathrm{s}} .67,+05^{\circ} 14^{\prime} 30^{\prime \prime}$. 1 . It is coincident with the $K_{S}=$ 11.6 mag source 2MASS J06392566+0514301.

1RXSJ150818.8-401730.-The Chandra source is pointlike and is at $15^{\mathrm{h}} 08^{\mathrm{m}} 18.17,-40^{\circ} 17^{\prime} 26^{\prime \prime} .0\left(8^{\prime \prime}\right.$ away from the BSC position). It is coincident with the $K_{s}=9.3 \mathrm{mag}$ source $2 \mathrm{MASS}$ J15081819-4017261.

1RXSJ150139.6-403815.-The Chandra source is extended. See $\oint$ 5.3.4.

1RXSJ151942.8-375255.-The Chandra source is pointlike and is at $15^{\mathrm{h}} 19^{\mathrm{m}} 42^{\mathrm{s}} .98,-37^{\circ} 52^{\prime} 51^{\prime \prime} .4$ ( $4^{\prime \prime}$ away from the BSC position). There is no $2 \mathrm{MASS}$ counterpart, but we do identify a counterpart on our Magellan MagIC optical and PANIC infrared observations ( $\S 5.2$ and Fig. 21). The source has $R=19.1 \mathrm{mag}$ and $K_{s}=15.79 \mathrm{mag}$. 
TABLE 3

Comments on the Identification of X-Ray Sources

\begin{tabular}{|c|c|c|c|c|c|}
\hline $\mathrm{ID}^{\mathrm{a}}$ & Number $^{b}$ & $\operatorname{Star}(\mathrm{s})^{\mathrm{c}}$ & Optical Figure(s) ${ }^{\mathrm{d}}$ & Chandra? ${ }^{\mathrm{e}}$ & Additional Notes ${ }^{\mathrm{f}}$ \\
\hline \multicolumn{6}{|l|}{ SNR G65.3+5.7: } \\
\hline $193445.6+303100 \ldots \ldots \ldots$ & 1 & HD 184738 & 7 & No & $\begin{array}{l}\text { Planetary nebula, associated with } 235.2 \text { mJy } \\
\text { NVSS source at } 19^{\mathrm{h}} 34^{\mathrm{m}} 45^{\mathrm{s}} .20 \\
\quad+30^{\circ} 30^{\prime} 58^{\prime \prime} .8 \text { (Condon \& Kaplan 1998) }\end{array}$ \\
\hline $193840.0+303035 \ldots \ldots \ldots$ & 2 & $\mathrm{~V}^{*}$ EM Cyg & 7 & No & $\begin{array}{l}\text { Dwarf nova; X-ray emission reported by } \\
\text { Richman (1996) and Verbunt et al. (1997) }\end{array}$ \\
\hline $193458.1+335301 \ldots \ldots .$. & 5 & & 7,25 & Yes & Extended Chandra source; see $\S 5.3 .1$ \\
\hline $192722.3+280934 \ldots \ldots .$. & 6 & USNO 1181-0406270 & 7 & No & \\
\hline $194401.5+284456 \ldots \ldots .$. & 7 & GSC 02151-03394 & 8 & No & Late type \\
\hline $194902.9+295258 \ldots \ldots .$. & 8 & HD 187460 & 8 & No & Pulsating variable star of type K2 II-III \\
\hline $193228.6+345318 \ldots \ldots . .$. & 9 & 2MASS J19322722+3453148 & 8,19 & Yes & $\begin{array}{l}35.4 \text { mJy NVSS counterpart at } 19^{\mathrm{h}} 32^{\mathrm{m}} 27^{\mathrm{s}} .20 \\
\quad+34^{\circ} 53^{\prime} 14^{\prime \prime} 8 \text {; flare star (Fuhrmeister \& } \\
\text { Schmitt } 2003 \text { ) }\end{array}$ \\
\hline $191151.1+285012 \ldots \ldots .$. & 14 & USNO 1188-0330651 & 9 & No & \\
\hline \multicolumn{6}{|l|}{ SNR G74.0-8.5: } \\
\hline $205042.9+284643 \ldots \ldots \ldots$ & 1 & $\ldots$ & 10 & Yes & Flare star? See $\S 5.3 .2$ \\
\hline $204457.4+291613 \ldots \ldots .$. & 2 & HD 335070 & 10 & No & Late type \\
\hline $205812.8+292037 \ldots \ldots \ldots$ & 3 & $\begin{array}{l}\text { USNO 1193-0519643, } \\
\text { 2MASS J20581257+2920454 }\end{array}$ & 10 & No & $\begin{array}{l}\text { Association may be somewhat questionable; } \\
\text { see } \S 5.3 .3\end{array}$ \\
\hline $205208.5+270546 \ldots \ldots \ldots$ & 4 & HD 198809 & 10 & No & Variable type G7 III star \\
\hline AX J2049.6+2939........ & $\ldots$ & 2MASS J20493540+2938509 & 19 & Yes & Late type \\
\hline \multicolumn{6}{|l|}{ SNR G156.2+5.7: } \\
\hline $050639.1+513607 \ldots \ldots$. & 1 & HD 32537 & 11 & No & Variable star of the $\gamma$ Dor type \\
\hline \multicolumn{6}{|l|}{ SNR G160.9+2.6: } \\
\hline $045707.4+452751 \ldots \ldots .$. & 1 & 2MASS J04570832+4527499 & 12,19 & Yes & \\
\hline $050339.8+451715 \ldots \ldots \ldots$ & 2 & 2MASS J05033958+4516594 & 12,19 & Yes & $\begin{array}{l}\text { Associated with the } 34.3 \text { mJy NVSS } \\
\text { source at } 05^{\mathrm{h}} 03^{\mathrm{m}} 39^{\mathrm{s}} .59 \\
\quad+45^{\circ} 16^{\prime} 58^{\prime \prime} 9 \text {; flare star? }\end{array}$ \\
\hline $064641.1+082152 \ldots \ldots \ldots$ & 4 & HD 49015 & 13 & No & Variable star of the $\gamma$ Dor type \\
\hline $062740.3+073103 \ldots \ldots$. & 5 & 2MASS J06274012+0731006 & 13,19 & Yes & \\
\hline $062937.2+082930 \ldots \ldots .$. & 6 & HD 45759 & 13 & No & Late type \\
\hline $063715.7+032005 \ldots \ldots .$. & 7 & $\begin{array}{l}\text { USNO 0150-00332-1, 2MASS } \\
\text { J06371585+0320043, } \\
\text { 2MASS J06371555+0320026 }\end{array}$ & 14 & No & Multiple-star system? \\
\hline $062554.8+065543 \ldots \ldots .$. & 8 & USNO 0145-01717-1 & 14 & No & \\
\hline 1E $0627.4+0537 \ldots \ldots \ldots . .$. & $\ldots$ & 2MASS J06300529+0545407 & 19 & Yes & \\
\hline 1E $0630.9+0611 \ldots \ldots \ldots . . .$. & $\ldots$ & 2MASS J06333322+0608396 & 20 & Yes & \\
\hline 1E $0636.8+0517 \ldots \ldots \ldots .$. & $\ldots$ & 2MASS J06392566+0514301 & 20 & Yes & \\
\hline \multicolumn{6}{|l|}{ SNR G330.0+15.0: } \\
\hline $150818.8-401730 \ldots \ldots$. & 1 & 2MASS J15081819-4017261 & 15,20 & Yes & $\begin{array}{l}\text { Also 1AXG J150818-4016 } \\
\text { (Ueda et al. 2001) }\end{array}$ \\
\hline $151059.6-392655 \ldots \ldots$. & 2 & $\begin{array}{l}\text { USNO 0505-0350285, USNO 7826-00179-1, } \\
\text { USNO 0505-0350290 }\end{array}$ & 15 & No & Multiple-star system? \\
\hline $150814.0-403445 \ldots \ldots$. & 3 & HD 133880 & 15 & No & $\begin{array}{l}\text { Variable star of the } \alpha^{2} \mathrm{CVn} \text { type; has } \\
\text { late-type companion } \\
\text { (Stelzer et al. 2003) }\end{array}$ \\
\hline $150428.9-392423 \ldots \ldots$. & 4 & CD-38 9913 & 15 & No & \\
\hline $150526.4-385709 \ldots \ldots$. & 5 & RX J1505.4-3857 & 15 & No & T Tauri star \\
\hline $150139.6-403815 \ldots \ldots .$. & 6 & 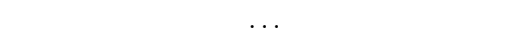 & 15,29 & Yes & Extended Chandra source; see $\S 5.3 .4$ \\
\hline $151849.8-405108 \ldots \ldots$. & 7 & $\mathrm{~V}^{*} \mathrm{LX}$ Lup & 16 & No & T Tauri star \\
\hline
\end{tabular}


TABLE 3-Continued

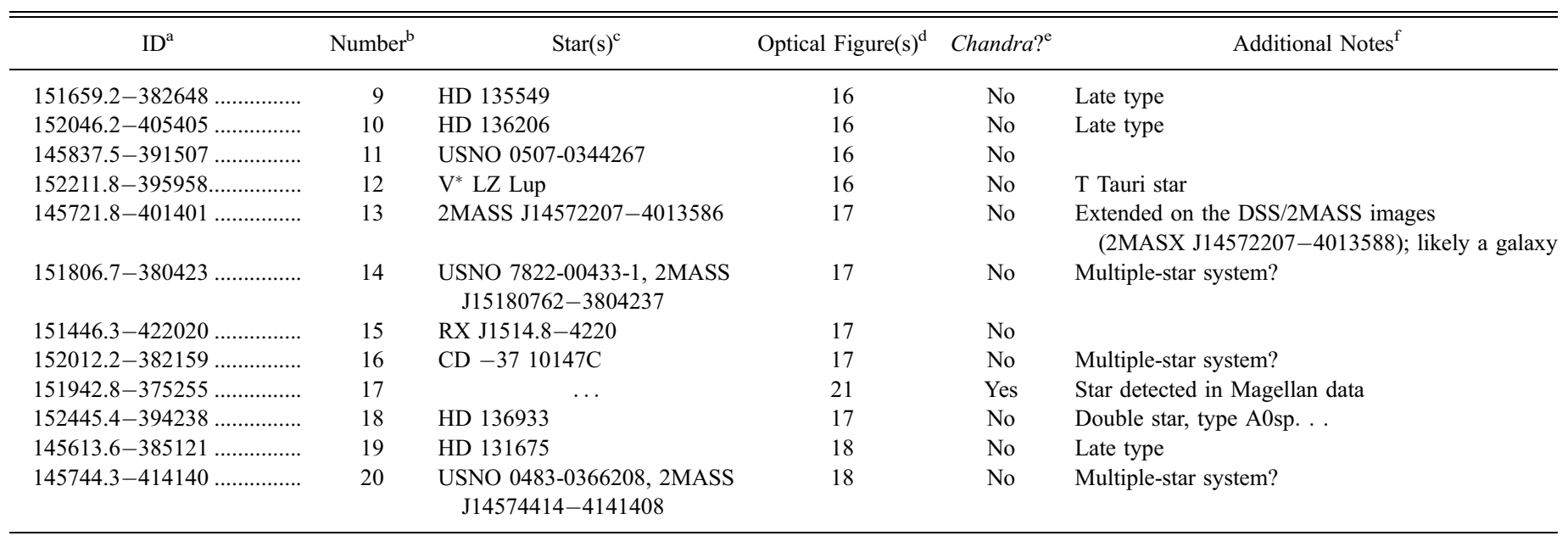

${ }^{\mathrm{a}} \mathrm{ID}$ of X-ray source, which is $1 \mathrm{RXS} \mathrm{J}$ unless otherwise indicated.

${ }^{b}$ Number of X-ray source in the given SNR from Table 2.

${ }^{\mathrm{c}}$ Name(s) of likely stellar companion(s). In contrast to Table 2, this also includes identifications made from Chandra follow-up observations.

${ }^{\mathrm{d}}$ Figure(s) where optical/IR counterparts are identified.

e Indicates whether source was selected for Chandra follow-up; see $\S 5.1$.

${ }^{f}$ Classifications are from SIMBAD unless otherwise noted. "Late type" means that the star is of type mid-F or later and hence is likely to have intrinsic X-ray emission (e.g., Stelzer et al. 2003).

\subsection{Optical and Infrared Follow-up}

We obtained optical and infrared follow-up observations of three of the four sources for which the Chandra follow-up did not immediately identify a counterpart, namely, 1RXS J193458.1+ 335301, 1RXS J151942.8-375255, and 1RXS J150139.6403815. For 1RXS J193458.1+335301, we got a $300 \mathrm{~s} g^{\prime}$-band exposure with the Large Format Camera (LFC) on the Palomar 200 inch $(5 \mathrm{~m})$ telescope and $1200 \mathrm{~s}$ exposures in $\mathrm{H} \alpha$ and a narrowband filter located away from major emission lines with the CCD camera (P60CCD) on the Palomar 60 inch $(1.5 \mathrm{~m})$ telescope. For 1RXS J151942.8-375255, we obtained a $930 \mathrm{~s} R$-band exposure with the Raymond and Beverly Sackler Magellan Instant Camera (MagIC) on the $6.5 \mathrm{~m}$ Clay (Magellan II) telescope, and a $200 \mathrm{~s} K_{s}$-band exposure with Persson's Auxiliary Nasmyth Infrared Camera (PANIC; Martini et al. 2004), also on the $6.5 \mathrm{~m}$ Clay
(Magellan II) telescope. For 1RXS J150139.6-403815, we got a $160 \mathrm{~s} B$-band exposure and a $60 \mathrm{~s} R$-band exposure with MagIC. The log of the observations is given in Table 5. Reduction and calibration followed standard procedures. The Magellan data were taken during the same observing runs as the data presented in Paper I, and details can be found there.

For the reduction of the remaining data, we used standard IRAF routines to subtract the bias, flat-field, and then combine separate exposures. Significant focal plane distortion prevented simple addition of the LFC data, so we used the IRAF MSCRED package to flatten each image with custom distortion maps prior to addition. We then performed absolute astrometry, solving for plate scale, rotation, and central position relative to stars in the 2MASS catalog, and getting residuals in each coordinate of $00^{\prime \prime} 13$ (2100 stars) and 0."17 (4000 stars) for the P60CCD and LFC, respectively.
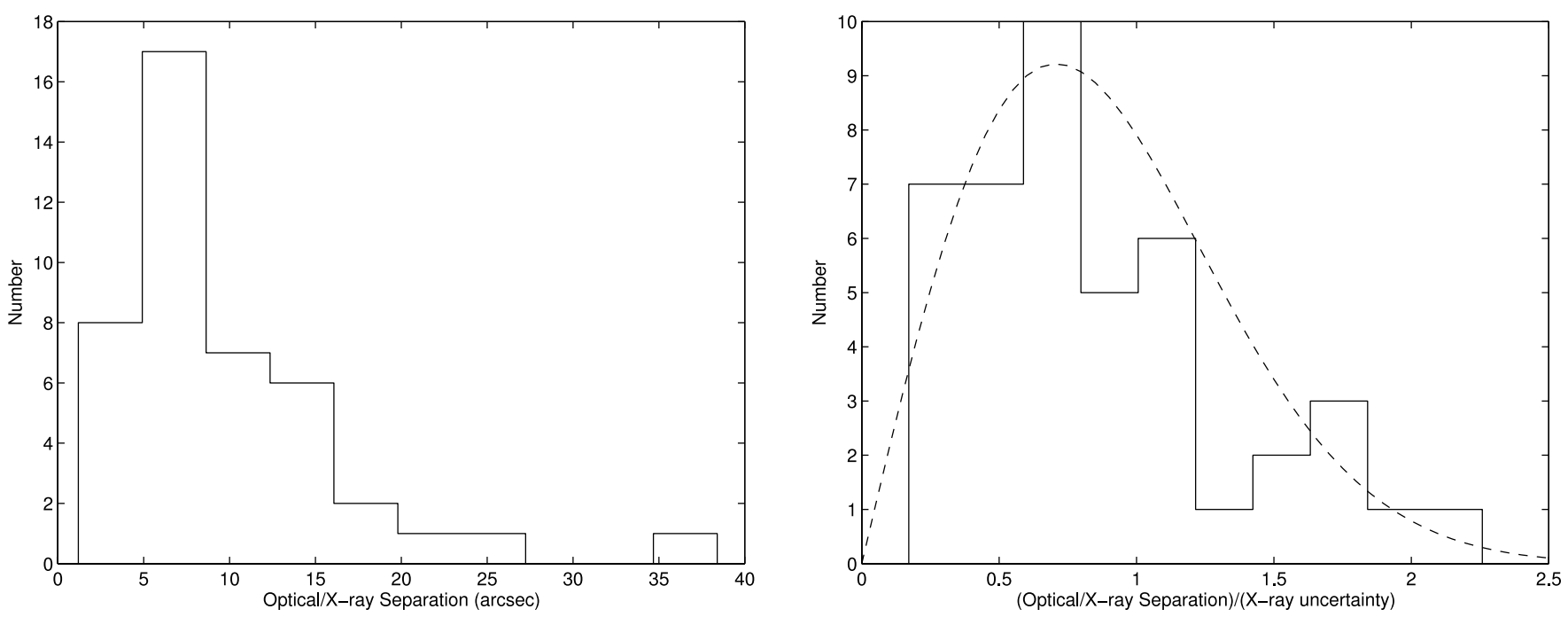

FIG. 4.-Distribution of the separation between the ROSAT positions and the positions of their optical counterparts. Left: Absolute separation in arcseconds. Right: Separation normalized to the X-ray position uncertainty, with the expected distribution for true associations $\left[f_{r} \propto r \exp \left(-r^{2}\right)\right.$, where $r$ is the normalized separation; dashed line] also plotted. This shows that we have largely identified the correct counterparts for the X-ray sources and that the position uncertainties are reasonable. 


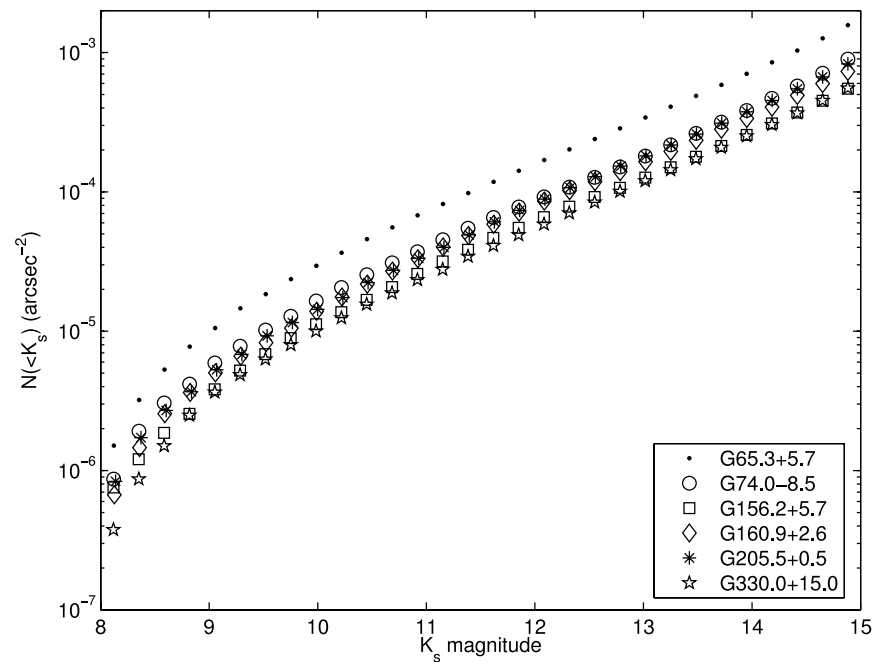

FIG. 5.-Cumulative number density of IR sources. The number of 2MASS sources per square arcsecond brighter than a given $K_{s}$ magnitude is plotted against $K_{s}$ magnitude for SNRs G65.3+5.7 (points), G74.0-8.5 (circles), G156.2+5.7 (squares), G160.9+2.6 (diamonds), G205.5+0.5 (asterisks), and G330.0+15.0 (stars). Typical PSPC error circles have radii of $\approx 10^{\prime \prime}$ (Table 2). [See the electronic edition of the Journal for a color version of this figure.]

\subsection{Remaining Sources}

We find that after investigating 50 BSC sources plus four sources from the literature and obtaining Chandra follow-up of 13 of these sources, there remain four X-ray sources that do not have very likely optical counterparts and are therefore worthy of extended discussion. As noted in $\S \S 4$ and 5.1, these sources are 1RXS J193458.1+335301, 1RXS J205042.9+284643, 1RXS J205812.8+292037, and 1RXS J150139.6-403815. The first and fourth have extended X-ray emission, while the second has no apparent Chandra counterpart. The third has a probable but not definite association with optical/IR sources. We now discuss all of these sources in more detail.

\subsection{1. $1 R X S J 193458.1+335301$}

The BSC lists 1RXS J193458.1+335301 as having $0.051 \pm$ 0.012 counts $\mathrm{s}^{-1}$ in the PSPC, with hardness ratios of HR1 = $1.00 \pm 0.17$ and HR2 $=0.10 \pm 0.23$ (see Voges et al. 1999 for definitions of bands and hardness ratios). The corresponding Chandra source is clearly extended, as shown in Figure 22. There are no other X-ray sources nearby, indicating that the Chandra source is very likely 1 RXS J193458.1+335301 despite the $\approx 32^{\prime \prime}$ distance between the two (this is somewhat larger than the separations between the optical and X-ray sources in Fig. 4, but given the extended nature of the X-ray source, this is not that surprising). The peak of the Chandra emission is at $19^{\mathrm{h}} 34^{\mathrm{m}} 55^{\mathrm{s}} .61$, $+33^{\circ} 53^{\prime} 06^{\prime \prime} 0$, and has an extent of $\approx 5^{\prime \prime}$. The overall source is larger and asymmetric, with a maximum visible distance of $\approx 40^{\prime \prime}$ from the peak to the northeast and a minimum distance of $\approx 15^{\prime \prime}$ from the peak to the southwest, although there is some diffuse emission that extends farther. Averaged over azimuth, the halfpower radius is $11^{\prime \prime}$, and $95 \%$ of the power is within $42^{\prime \prime}$. Fitting the spatial profile to a $\beta$-model (surface brightness $\propto[1+$ $\left.\left(r / r_{c}\right)^{2}\right]^{-3 \beta+0.5}$, typical for galaxy clusters) in Sherpa was successful, with core radius $r_{c}=33^{\prime \prime} 5 \pm 0$ " $1, \beta=0.451 \pm 0.005$, an amplitude of $0.64 \pm 0.03$ counts pixel ${ }^{-2}$, and $\chi^{2}=9.6$ for 12 degrees of freedom.

We extracted photon events from a $45^{\prime \prime} \times 22^{\prime \prime}$ region and created source and background response files using the CIAO task acisspec. We used versions 3.0.2 of CIAO and 2.26 of

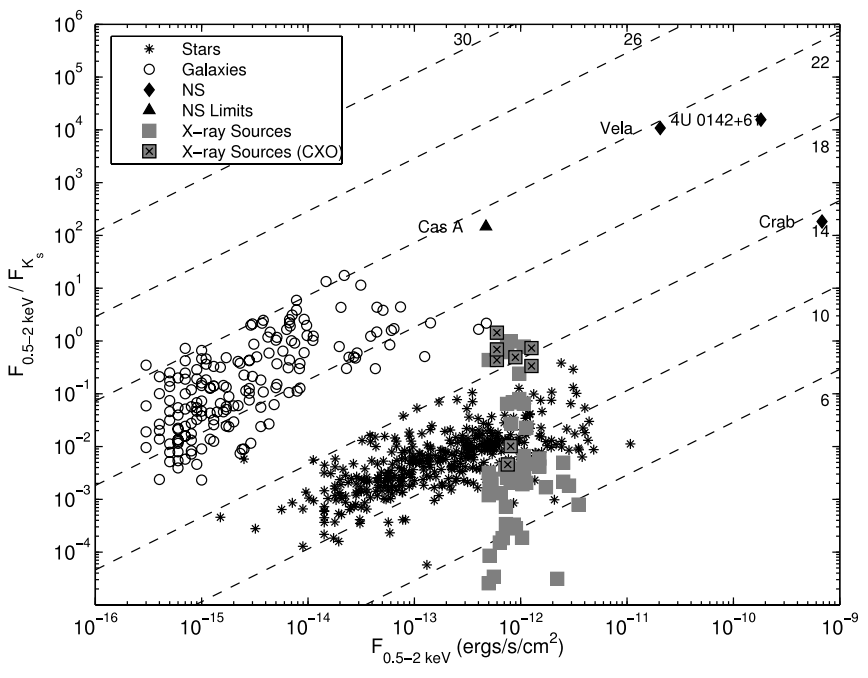

FIG. 6.-X-ray-to $-K_{s}$ flux ratio vs. X-ray flux for sources from Table 2, with sources from the CDF/Orion studies and selected neutron stars. Stars from $\mathrm{CDF} /$ Orion are shown as asterisks, and galaxies are shown as circles. Selected neutron stars are shown by the black diamonds/limits and are labeled. The X-ray sources from Table 2 (including detections from $\S 5.1$ ) are shown by the squares (those with Chandra follow-up, plus 1RXS J205812.8+292037, have crosses in their squares). The diagonal lines represent constant magnitude and are labeled by that magnitude. For the X-ray sources from Table 2, the PSPC values were converted to a flux by $F_{0.5}-2.0 \mathrm{keV}=\mathrm{PSPC} \times 1 \times 10^{-11} \mathrm{ergs} \mathrm{s}^{-1} \mathrm{~cm}^{-2}$, appropriate for a blackbody with $k T_{\infty}=0.25 \mathrm{keV}$ and $N_{\mathrm{H}}=5 \times 10^{20} \mathrm{~cm}^{-2}$. The $\mathrm{X}$-ray source are largely consistent with foreground stars, especially considering the possible range of temperatures and column densities, but a number may also be active galaxies such as those found by Rutledge et al. (2003). [See the electronic edition of the Journal for a color version of this figure.]

CALDB, which compensate for the low-energy degradation of the ACIS detector. ${ }^{8}$ We then fit the data in Sherpa. The events were binned so that each bin had $\geq 25$ counts.

While there are not very many counts (601 source counts before background subtraction, with 77.2 background counts), the data are well fit (Fig. 23) by an absorbed power-law model, with $N_{\mathrm{H}}=(3.0 \pm 0.6) \times 10^{21} \mathrm{~cm}^{-2}, \Gamma=2.4 \pm 0.2$, and an amplitude of $(4.6 \pm 0.8) \times 10^{-4}$ photons cm $\mathrm{cm}^{-2} \mathrm{~s}^{-1} \mathrm{keV}^{-1}$ at $1 \mathrm{keV}$ (giving $\chi^{2}=18.7$ for 16 degrees of freedom; all uncertainties are $1 \sigma$ ). The observed flux from this model is $1.0 \times 10^{-12} \mathrm{ergs} \mathrm{cm}^{-2}$ $\mathrm{s}^{-1}(0.3-8.0 \mathrm{keV})$, while the unabsorbed flux is $2.2 \times 10^{-12} \mathrm{ergs}$ $\mathrm{cm}^{-2} \mathrm{~s}^{-1}(0.3-8.0 \mathrm{keV})$. Raymond \& Smith (1977) plasma models do not fit, giving $\chi_{\nu}^{2}=70 / 16$.

The extended morphology and the hard spectrum of the source suggest several models: (1) a very hot nebula of Galactic origin, (2) a very hot nebula but of extragalactic origin (gas from a cluster or an early-type galaxy; Fabbiano 1989; Brown \& Bregman 1998), (3) the superposition of many bright low-mass X-ray binaries (with power-law spectra) in an early-type galaxy (Matsushita et al. 1994), or (4) a PWN radiating via synchrotron emission. Here we discuss each interpretation in some detail.

Galactic nebula.-This would require a very bright central source (i.e., an OB star) to heat the nebula, which would also be visible as an extended optical/IR source and should show $\mathrm{H} \alpha$ emission. While the northern 2MASS source (2MASS $\mathrm{J} 19345557+3353136$ ) in Figure 25 is moderately bright (with about a $5 \%$ chance of a star this bright occurring randomly within $7^{\prime \prime}$ of a position), it does not appear to be an OB star. The colors are fairly common for the field, they are more consistent with a star of type $\approx \mathrm{G} 0$, and the extinction that would be required of an $\mathrm{OB}$ star is higher than expected for this line of sight

\footnotetext{
${ }^{8}$ See http://cxc.harvard.edu/ciao/threads/acisapplycti.
} 

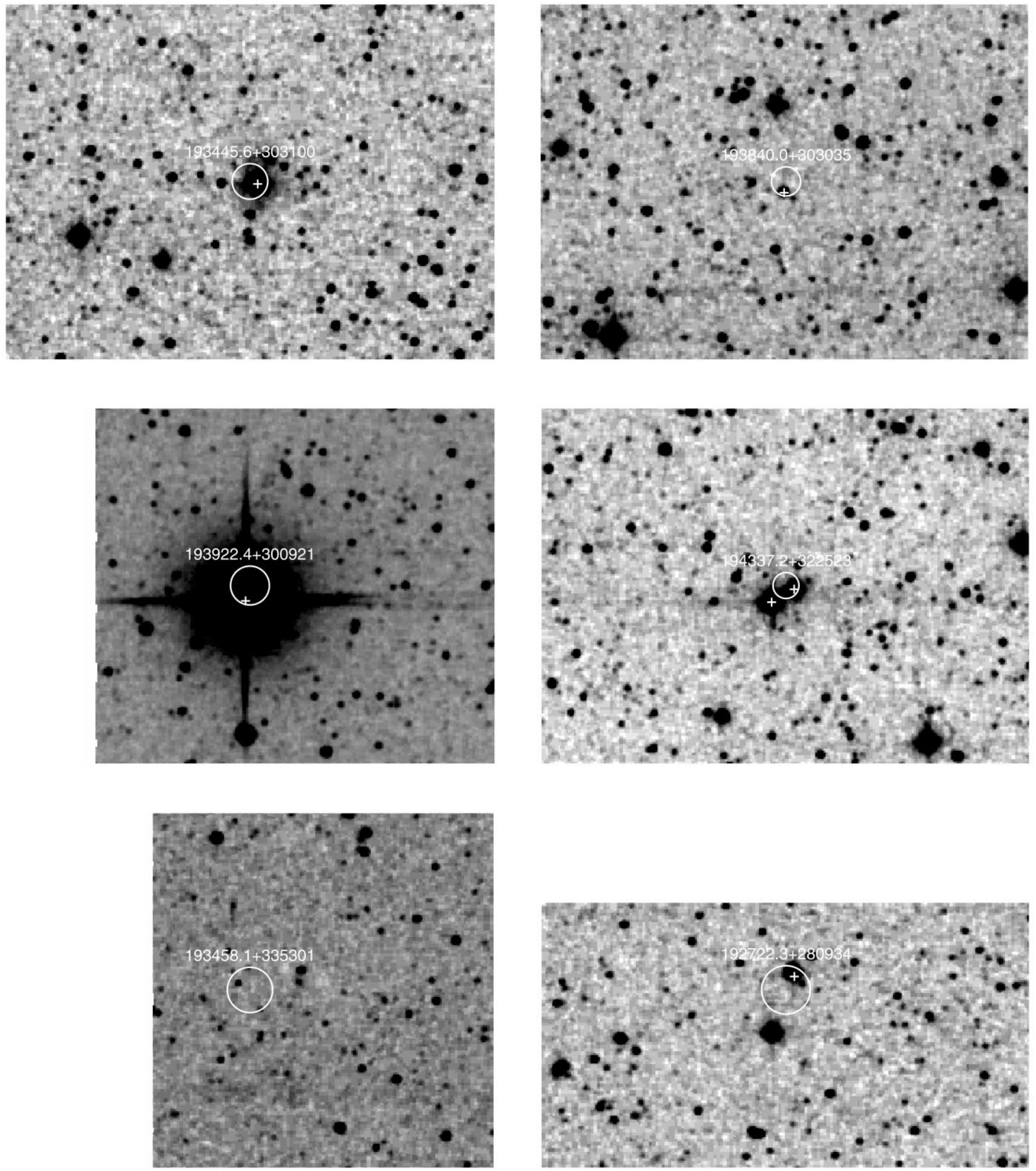

FIG. 7.-2MASS $K_{\mathrm{s}}$-band images of the sources in SNR G65.3+5.7 from Table 2. The images are $5^{\prime} \times 3.5$, with north up and east to the left. The X-ray position uncertainties are indicated by the circles, and the proposed optical counterparts are shown by the plus signs. [See the electronic edition of the Journal for a color version of this figure.]

( $A_{V} \gtrsim 2$ mag, compared to a maximum of $A_{V} \approx 1$ as determined by Drimmel et al. 2003 and $\mathrm{W} 3 \mathrm{COLDEN}^{9}$ ). The southern 2MASS source (2MASS J19345569+3353063) is fainter and redder, consistent with being a late-type star, but its position near the peak of the X-ray emission (chance probability of $<1 \%$ ) sug-

\footnotetext{
${ }^{9}$ See http://asc.harvard.edu/toolkit/colden.jsp.
}

gests that there might be an actual association between it and the $\mathrm{X}$-ray emission. Since there is no diffuse broadband optical, broadband IR, or $\mathrm{H} \alpha$ emission, we do not believe that the extended X-ray emission is powered by any of the stars, although it may be related to one or both of the 2MASS sources.

Extragalactic nebula.-The X-ray emission is much more compact than is typical for galaxy groups or clusters (even 

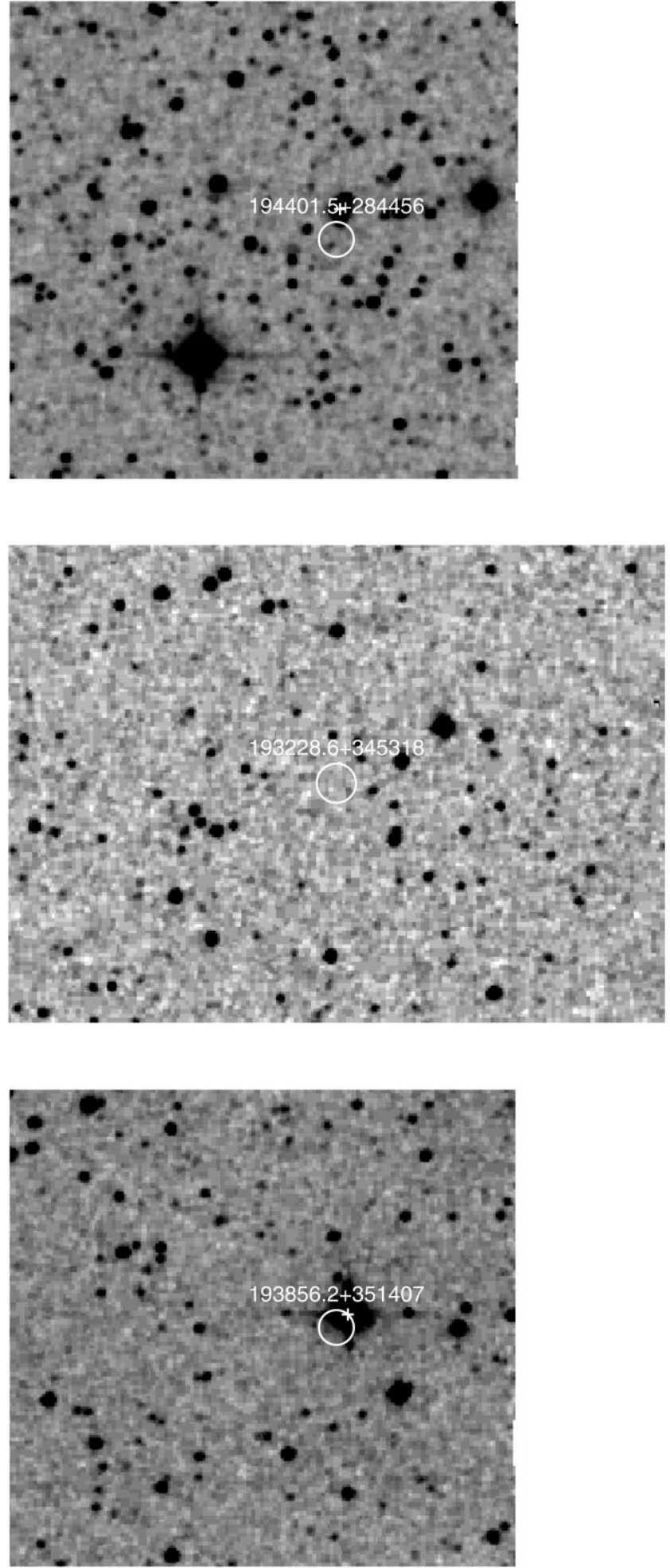
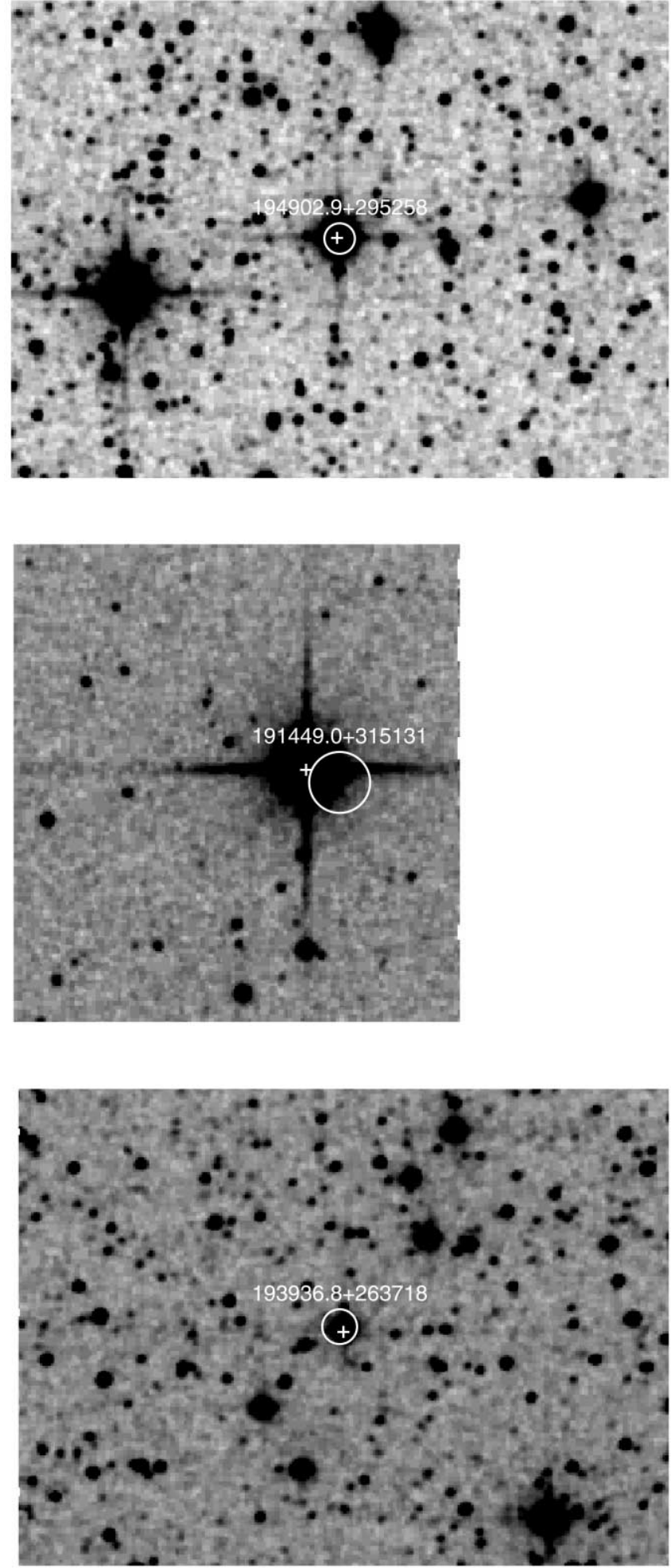

FIG. 8.-Additional 2MASS $K_{s}$-band images of the sources in SNR G65.3+5.7 from Table 2. [See the electronic edition of the Journal for a color version of this figure.]

clusters at $z \sim 0.5-1$ have $r_{c} \gg 10^{\prime \prime}$; Arnaud et al. 2002; Cotter et al. 2002), and the spectrum is wrong (thermal plasma models do not fit).

Early-type galaxy.-The size is similar to what is often seen for early-type ( $\mathrm{E}$ and $\mathrm{S} 0$ ) galaxies. In these galaxies the X-ray emission comes from a combination of hot gas (plasma with $k T \approx 0.5-1.0 \mathrm{keV}$; Fabbiano 1989; Brown \& Bregman 1998) and the superposition of many hard $\mathrm{X}$-ray point sources (whose spectra are power laws with $\Gamma \approx 1.7$ ), which is reasonably compatible with the observed spectrum of 1RXS J193458.1+335301.

For these galaxies, the hard X-ray luminosity scales reasonably well with the integrated $B$ - or $K$-band luminosities as 

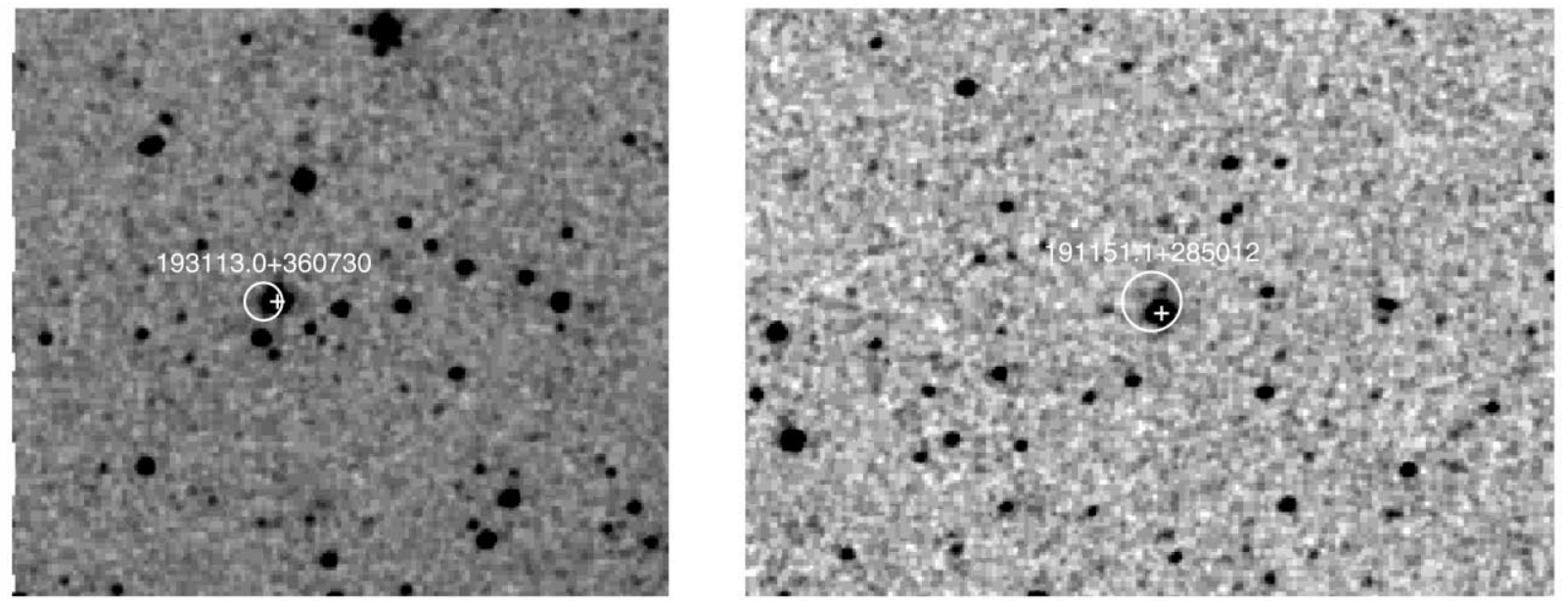

FIG. 9.-Additional 2MASS $K_{s}$-band images of the sources in SNR G65.3+5.7 from Table 2. [See the electronic edition of the Journal for a color version of this figure.]

$L_{\mathrm{X}} / L_{B} \approx 10^{30} \mathrm{ergs} \mathrm{s}^{-1} L_{\odot, B}^{-1}$ or $L_{\mathrm{X}} / L_{K} \approx 10^{29} \mathrm{ergs} \mathrm{s}^{-1} L_{\odot, K}^{-1}$, where the X-ray luminosity is in the $0.3-8.0 \mathrm{keV}$ band and all luminosities are corrected for extinction (Kim \& Fabbiano 2004); the scatter in this (from a sample of 14 galaxies) is a factor of 2-3 (there is more scatter in Brown \& Bregman 1998, but their work concerns the soft emission more than the hard emission, and the scatter is still only a factor of $\sim 10$ ). We can convert the rela- tions from Kim \& Fabbiano (2004) into relations for fluxes and magnitudes (i.e., observables), giving

$$
\begin{aligned}
& \frac{L_{\mathrm{X}}}{L_{B}}=4 \pi \times 10^{39} F_{\mathrm{X}} 10^{\left(m_{B}-M_{\odot, B}-N_{\mathrm{H}, 21} / 1.3\right) / 2.5} \mathrm{ergs} \mathrm{s}^{-1} L_{\odot, B}^{-1}, \\
& \frac{L_{\mathrm{X}}}{L_{K}}=4 \pi \times 10^{39} F_{\mathrm{X}} 10^{\left(m_{K}-M_{\odot, K}-N_{\mathrm{H}, 21} / 16\right) / 2.5} \operatorname{ergs~s}^{-1} L_{\odot, K}^{-1},
\end{aligned}
$$
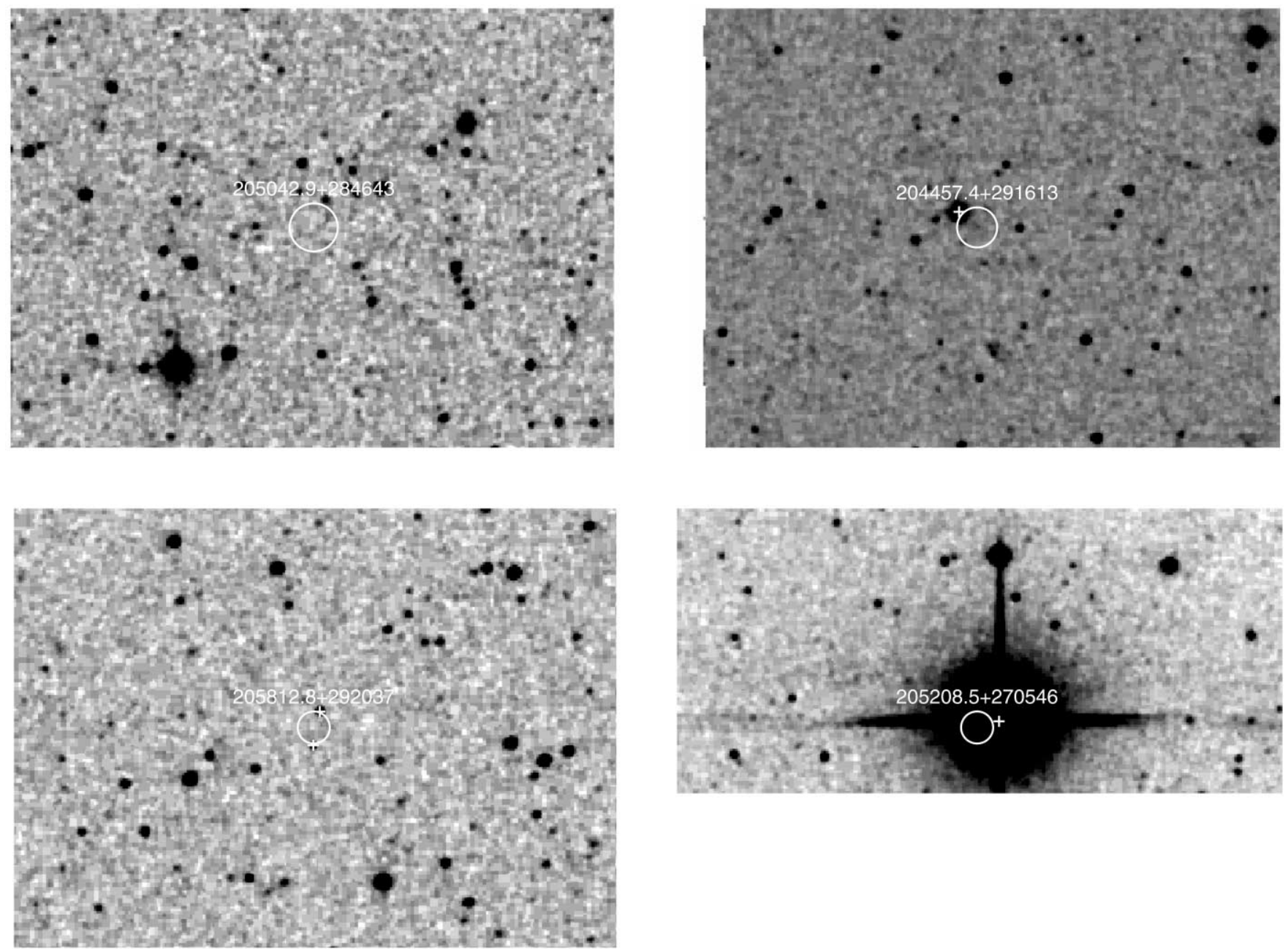

FIG. 10.-2MASS $K_{\mathrm{s}}$-band images of the sources in SNR G74.0 - 8.5 from Table 2. The images are $5^{\prime} \times 3.5$, with north up and east to the left. The X-ray position uncertainties are indicated by the circles, and the proposed optical counterparts are shown by the plus signs. [See the electronic edition of the Journal for a color version of this figure.] 


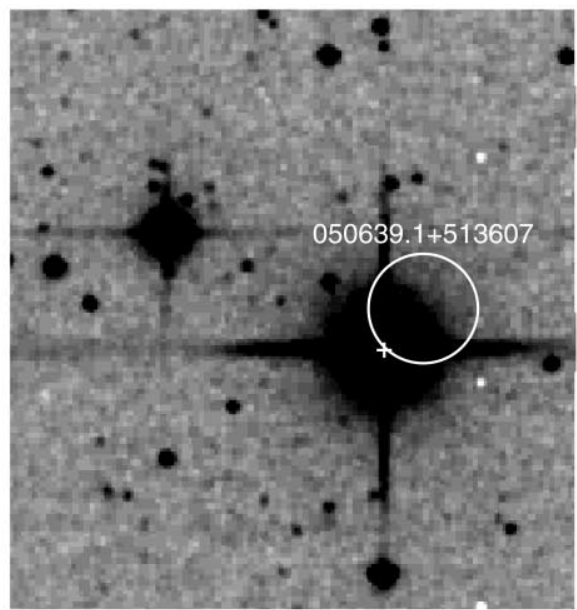

FIG. 11.-2MASS $K_{s}$-band image of the source in SNR G156.2+5.7 from Table 2 . The image is $5^{\prime} \times 3.5$, with north up and east to the left. The X-ray position uncertainty is indicated by the circle, and the proposed optical counterpart is shown by the plus sign. [See the electronic edition of the Journal for a color version of this figure.] where $F_{\mathrm{X}}$ is the absorption-corrected X-ray flux in the $0.3-$ $8.0 \mathrm{keV}$ band in units of ergs s $\mathrm{cm}^{-1}, m_{B}\left(m_{K}\right)$ is the observed $B$-band ( $K$-band) magnitude, $N_{\mathrm{H}, 21}$ is the Galactic column density along the line of sight in units of $10^{21} \mathrm{~cm}^{-2}$, and $M_{\odot, B}=$ $5.47 \mathrm{mag}\left(M_{\odot, K}=3.33 \mathrm{mag}\right)$ is the $B$-band ( $K$-band) absolute magnitude of the Sun (we have assumed $N_{\mathrm{H}, 21}=1.79 A_{V}$ following Predehl \& Schmitt 1995).

The relation in equation (1) implies that for $F_{\mathrm{X}}=10^{-12} \mathrm{ergs} \mathrm{s}^{-1}$ $\mathrm{cm}^{-2}$ and $N_{\mathrm{H}, 21}=1, m_{B} \approx 12 \mathrm{mag}$ and $m_{K} \approx 5 \mathrm{mag}$ (this indeed matches what is seen for sources in Kim \& Fabbiano 2004). Therefore, such sources should be readily visible on even shallow images. Examining 2MASS and Palomar (Fig. 25) images of 1RXS J193458.1+335301, we see that there are two optical/IR sources near the peak of the X-ray emission: the northern source appears stellar $(\mathrm{FWHM} \approx 1$."4), while the southern source may have some extended emission ${ }^{10}$ to the northeast (although this could be a superposition of point sources). However, neither of these sources is a great candidate for the origin of the X-ray emission, as they are too faint by several orders of magnitude $\left(K_{s}=\right.$ $14.4 \mathrm{mag}$ and $K_{s}=12.6 \mathrm{mag}$ for the northern and southern sources, respectively) and not extended enough. Therefore, while it is not impossible that 1RXS J193458.1+335301 is an early-type galaxy,

10 There are no data from the 2MASS Extended Source Catalog in this region owing to the presence of a bright $(V=6.7 \mathrm{mag}) \mathrm{M}$ star $90^{\prime \prime}$ to the south.
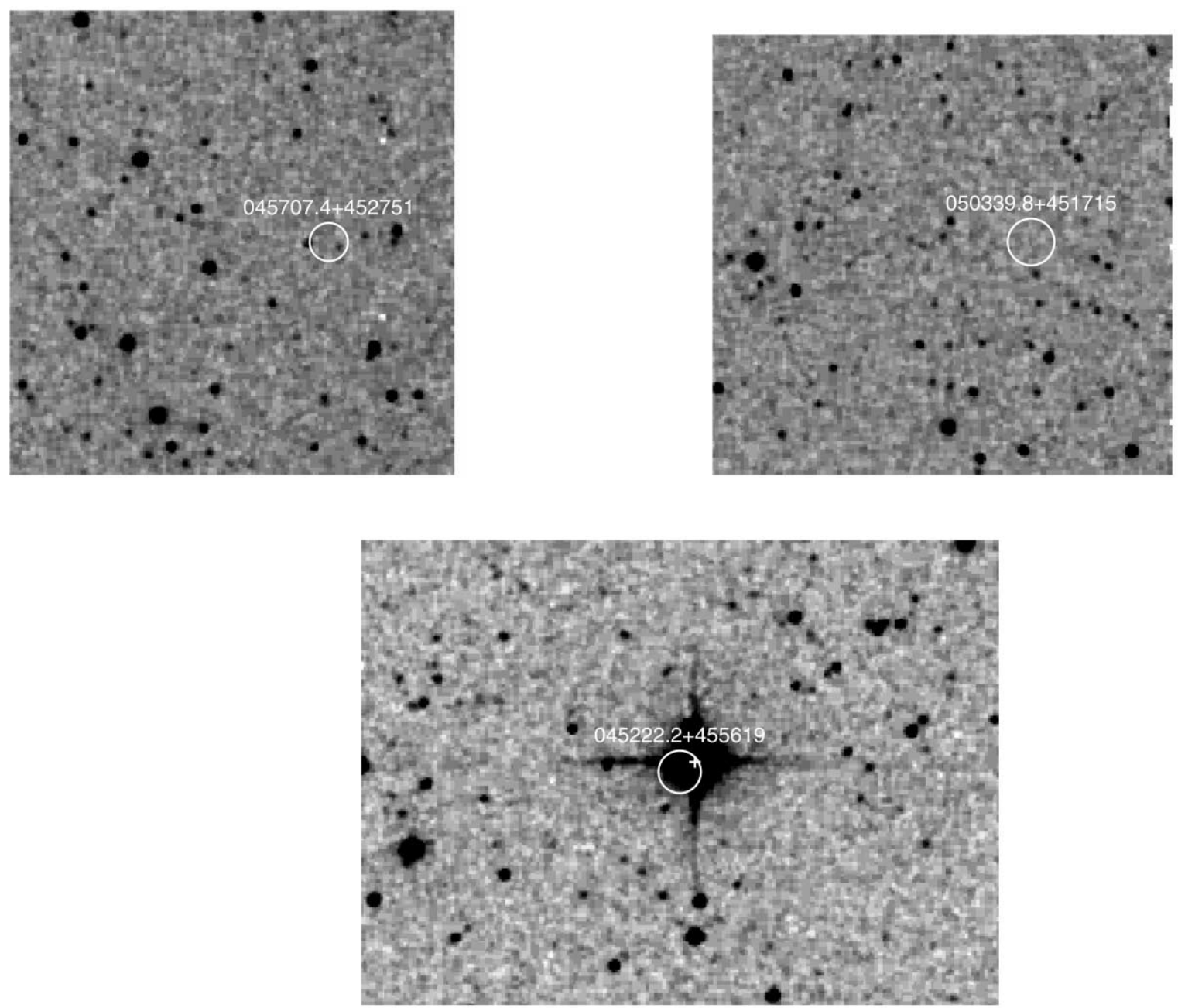

FIg. 12.-2MASS $K_{\mathrm{s}}$-band images of the sources in SNR G160.9+2.6 from Table 2. The images are $5^{\prime} \times 3.5$, with north up and east to the left. The X-ray position uncertainties are indicated by the circles, and the proposed optical counterparts are shown by the plus signs. [See the electronic edition of the Journal for a color version of this figure.] 

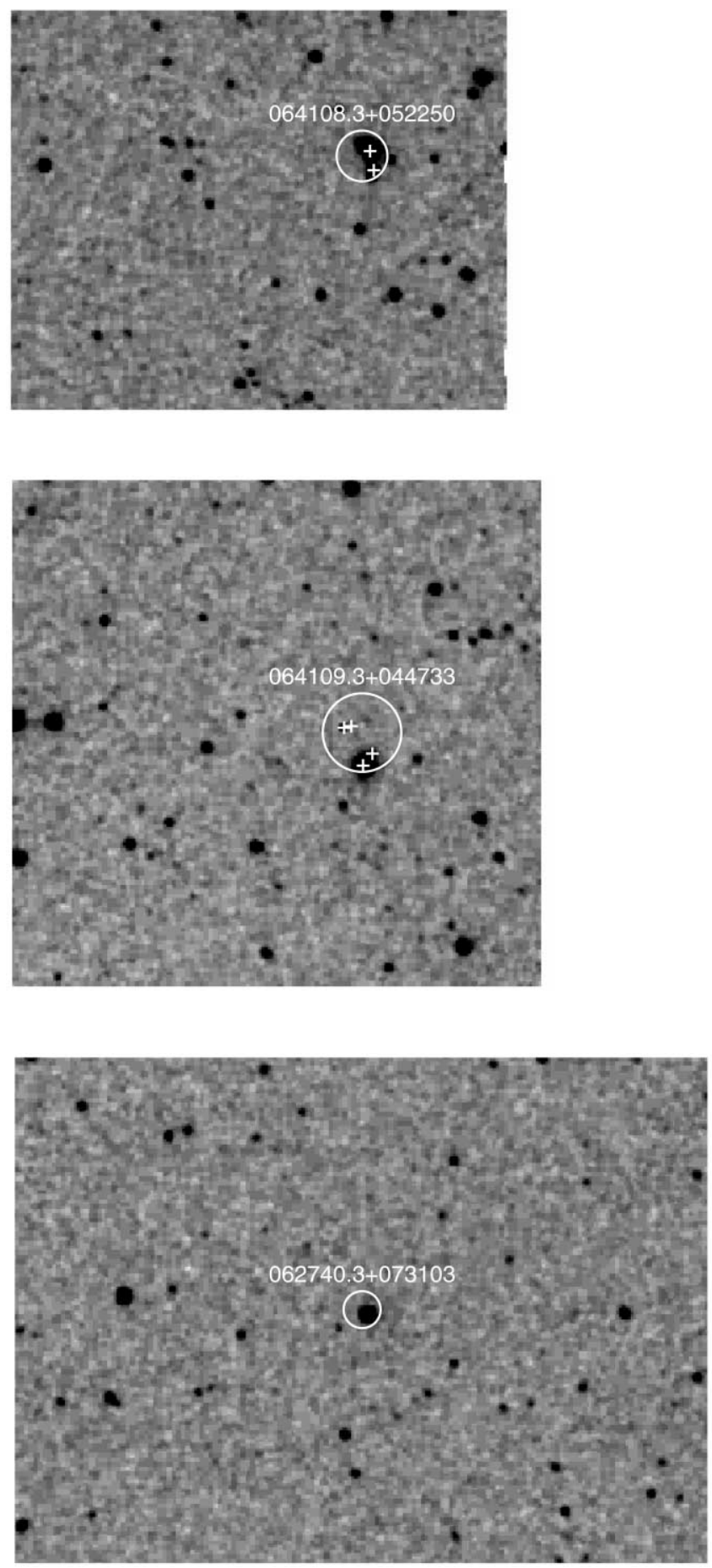

FIG. 13.-2MASS $K_{s}$-band images of the sources in SNR G205.5+0.5 from Table 2 . The images are $5^{\prime} \times 3$. $^{\prime} 5$, with north up and east to the left. The X-ray position

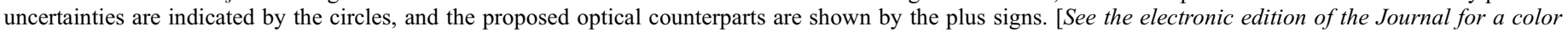
version of this figure.]

we consider it unlikely. Deeper X-ray observations should be conclusive; if 1RXS J193458.1+335301 is a galaxy, it should resolve into discrete point sources. Optical spectroscopy would also be useful in determining the natures of the optical/IR sources.

There are some early-type galaxies with significant excesses of X-ray emission (Vikhlinin et al. 1999b), largely due to increases in the amounts of hot gas that give roughly the same
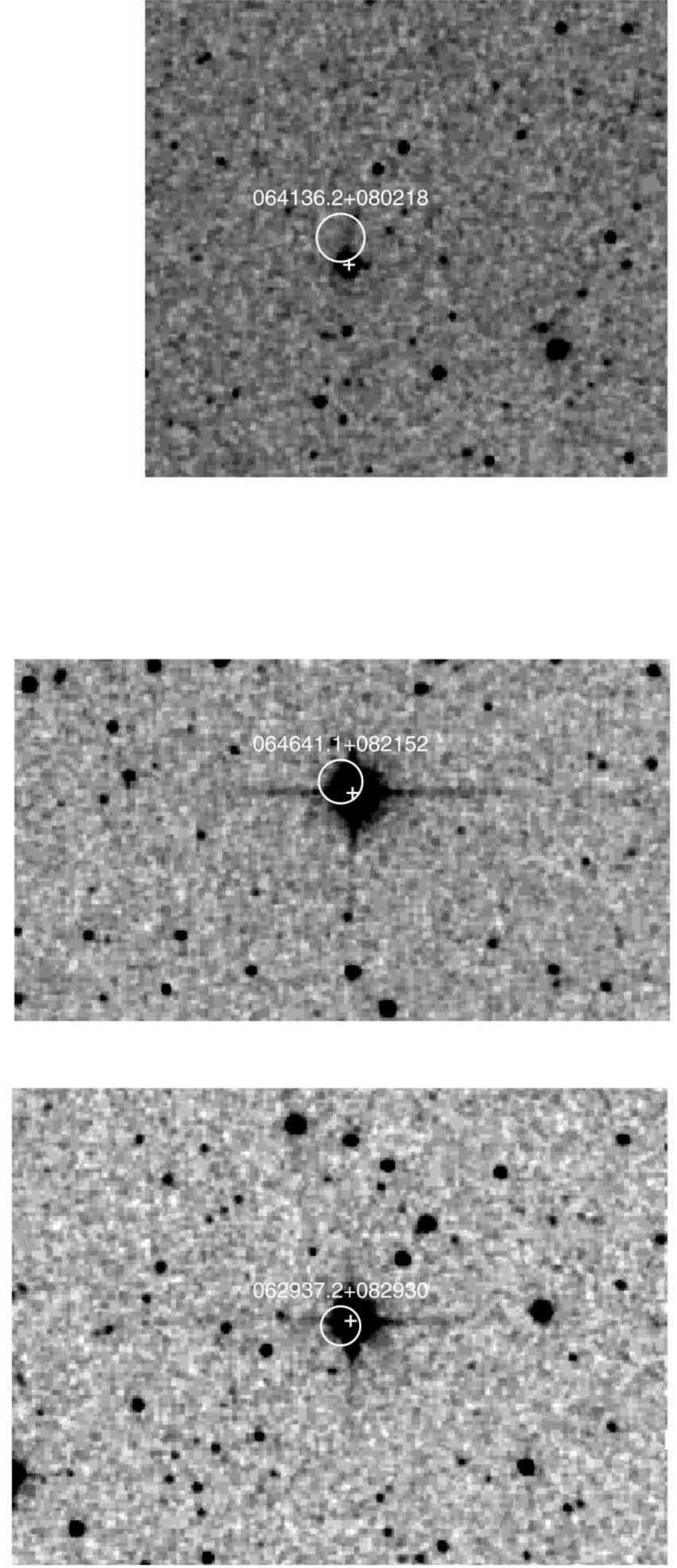

X-ray-to-optical ratio as would be necessary here. However, the optical/IR sources in Figure 25 do not look like bright galaxies (unlike the galaxies from Vikhlinin et al. 1999b, which are typically $>20^{\prime \prime}$ in the optical) and the spectrum of 1RXS J193458.1+ 335301 is wrong; again, thermal plasma models do not fit.

$P W N$.- A pulsar wind nebula (i.e., a nebula excited by a pulsar; for a review, see Gaensler \& Slane 2006) is consistent with 

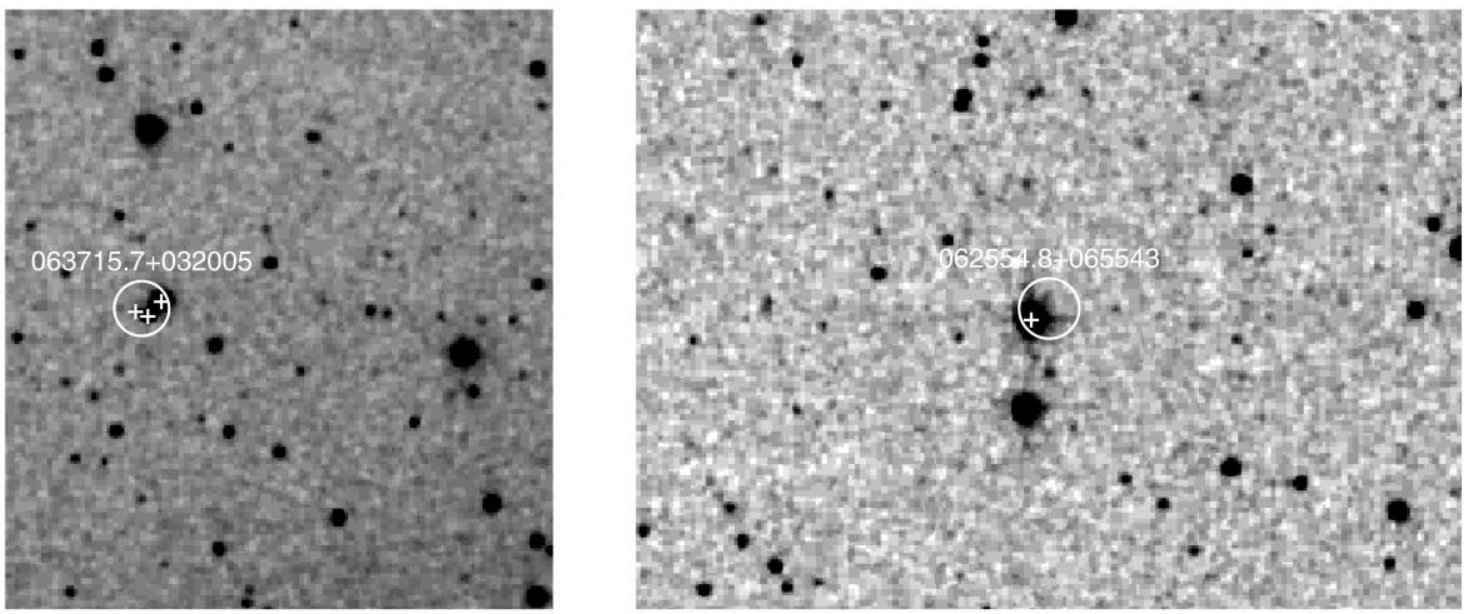

FIg. 14.-Additional 2MASS $K_{s}$-band images of the sources in SNR G205.5+0.5 from Table 2. [See the electronic edition of the Journal for a color version of this figure.]

the size and spectrum of 1RXS J193458.1+335301, although the source is slightly softer toward the center (Fig. 24), contrary to what is expected for PWNe. There is no obvious $\mathrm{H} \alpha$ emission from 1RXS J193458.1+335301 in Figure 25, as there can be near PWNe (associated either with SN ejecta or with the passage of the pulsar through the interstellar medium; Hester et al. 1990; Chatterjee \& Cordes 2002), but this could be because the conditions are not favorable.

1RXS J193458.1+335301 is outside SNR G65.3+5.7. If it were a bow shock nebula that originated in the interior of the SNR and then moved outside the shell (and not a static PWN inflated by the wind of its central pulsar), one would expect $\mathrm{H} \alpha$ emission and that the X-ray nebula would trail away from the direction of motion/toward the SNR center (e.g., Stappers et al. 2003 , although this is not always the case). Since the X-ray emission trails away from the SNR center (suggesting motion toward the SNR instead of out of it), since we see no $\mathrm{H} \alpha$, and since the fitted value of $N_{\mathrm{H}}$ is just about at the maximum predicted for this line of sight by W3COLDEN and somewhat higher than the nominal value for SNR G65.3+5.7 (suggesting that the X-ray source may be more distant and highly absorbed than SNR G65.3+5.7), we believe that an association between the two is unlikely. However, this is not entirely unexpected, as there are a number of young, newly discovered PWNe that have no definitively associated SNRs (similar to the Crab Nebula; Seward et al. 2006).

\subsection{2. $1 R X S J 205042.9+284643$}

The BSC lists 1RXS J205042.9+284643 as having $0.11 \pm$ 0.02 counts $\mathrm{s}^{-1}$ in the PSPC, with hardness ratios of HR1 = $-0.03 \pm 0.17$ and HR2 $=-0.87 \pm 0.14$. The Chandra observation of 1 RXS J205042.9+284643 had a total exposure time of $3.7 \mathrm{ks}$, which should give $\approx 1000$ ACIS-S counts, depending on the source spectrum. However, as shown in Figure 26 there are no point sources detected anywhere within 3 times the nominal position uncertainty (a conservative limit, as seen in Fig. 4); the only significant source detected (using wavdetect on scales from 1 to 32 pixels; see Paper I for the detection method) in the data set is at $20^{\mathrm{h}} 50^{\mathrm{m}} 39^{\mathrm{s}} .01,+28^{\circ} 45^{\prime} 43^{\prime \prime} .6$ (with $12 \pm 3$ counts), which is $79^{\prime \prime}$ or $6 \sigma$ away from 1RXS J205042.9+284643. This X-ray source is almost certainly not related to $1 \mathrm{RXS} \mathrm{J} 205042.9+$ 284643 . We can then set a limit of $\approx 3$ counts to any point source. There are no obvious extended sources, but such limits are more difficult to quantify; overall, there are 1047 counts in the $0.3-$ $5.0 \mathrm{keV}$ energy range over the whole $512^{\prime \prime} \times 128^{\prime \prime}$ image, so the average background rate is $0.0160 \pm 0.0005 \operatorname{arcsec}^{-2}$. Thus, in a region $\theta \times \theta \operatorname{arcsec}^{2}$ in area, the $3 \sigma$ limits would be $3\left[0.016 \theta^{2}+\right.$ $\left.\left(3 \times 10^{-7}\right) \theta^{4}\right]^{1 / 2}$ counts. There are no regions in the event list with such concentrations, so no extended sources are present.

One obvious explanation of the disappearance of $1 \mathrm{RXS}$ $\mathrm{J} 205042.9+284643$ is variability. This is not atypical among the most common class of soft X-ray sources in the Galactic plane: active stars. Flares and other chromospheric/magnetospheric events often lead to dramatic changes in the fluxes of these sources. While other sources, such as X-ray binaries, active galaxies, and some AXPs, do exhibit variability, these sources have hard X-ray spectra generally inconsistent with the BSC emission. We therefore think it likely that 1RXS J205042.9+284643 is an active star, but of course this cannot be confirmed without additional data. It is also possible that the source is extended and therefore too diffuse to have been detected by Chandra. The softness of the BSC emission makes this unlikely, however, as most known types of extended sources are relatively hard (e.g., 1RXS J193458.1+335301 and 1RXS J150139.6-403815).

It is possible, but unlikely, that 1RXS J205042.9+284643 is a neutron star. As discussed above, most of the neutron stars considered in Paper I have stable X-ray emission; only some of the AXPs vary significantly. However, the spectrum of 1RXS J205042.9+ 284643 is unlike those of AXPs (typically power laws with $\Gamma \sim 3)$.

\subsection{3. $1 R X S J 205812.8+292037$}

The BSC lists 1RXS J205812.8+292037 as having $0.13 \pm$ 0.02 counts $\mathrm{s}^{-1}$ in the PSPC, with hardness ratios of $\mathrm{HR} 1=$ $0.63 \pm 0.11$ and HR2 $=0.27 \pm 0.13$. This is moderately hard compared to the other sources in Figure 1, but not too extreme.

Unlike the rest of the sources without Chandra follow-up, the counterpart(s) shown in Figure 10 are not entirely secure. Within $10^{\prime}$ of 1RXS J205812.8+292037, there are 1627 2MASS sources, for an average density of $(1.44 \pm 0.04) \times 10^{-3} \operatorname{arcsec}^{-2}$. Finding a source within $9^{\prime \prime}$ (as in the case of 1RXS J205812.8+292037) has a chance probability of $37 \%$, and the chance probability for two sources is $13 \%$. These are not small enough for a definite association. 1RXS J205812.8+292037 is similar, in both hardness ratio and optical brightness, to other sources such as 1RXS J193228.6+345318, 1RXS J205042.9+284643, 1RXS J045707.4+ 452751, or 1RXS J151942.8-375255. These sources did not have certain associations based on ROSAT alone, but the Chandra data are unambiguous. These sources may represent a population of 

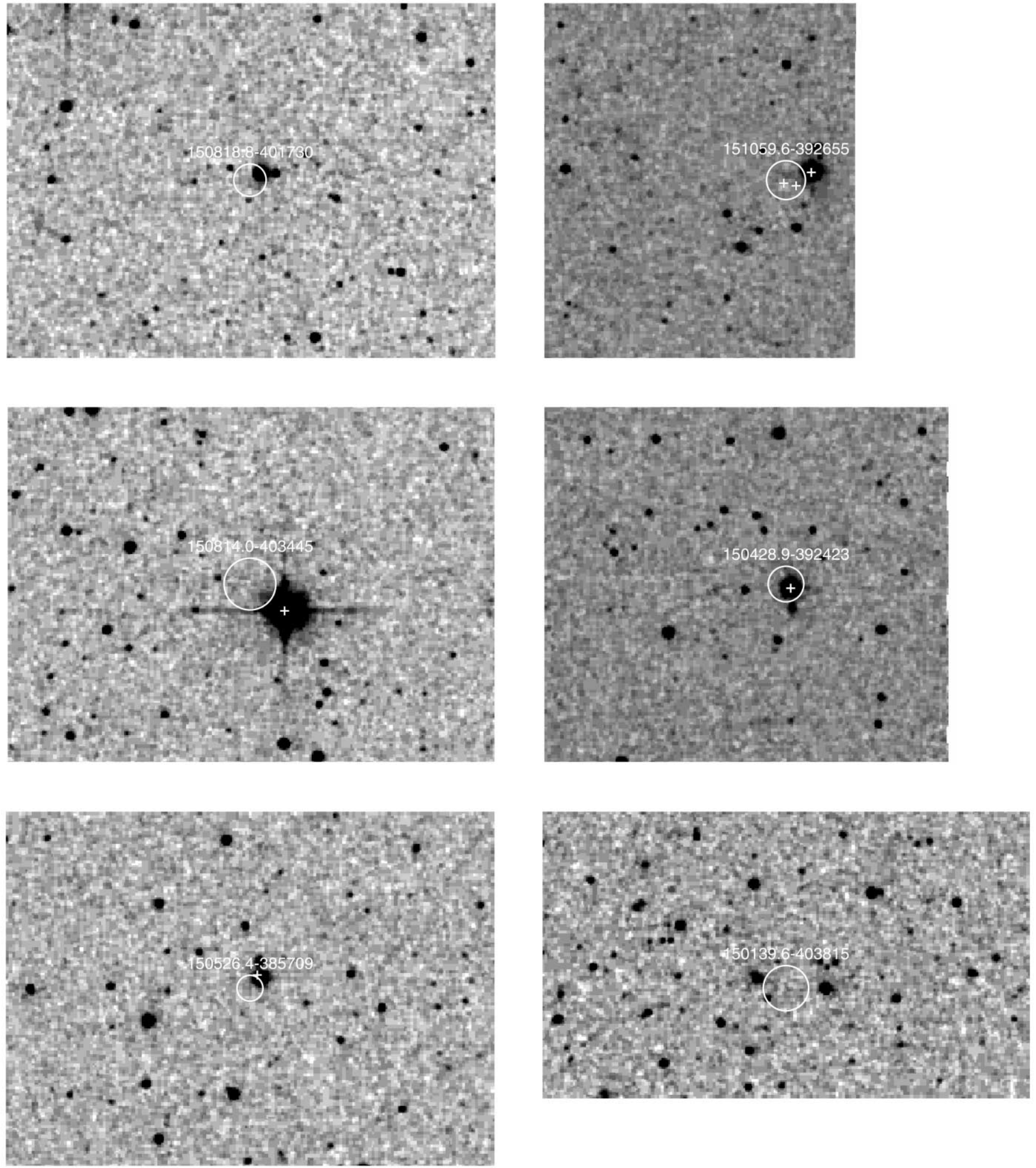

FIG. 15.-2MASS $K_{s}$-band images of the sources in SNR G330.0+15.0 from Table 2 . The images are $5^{\prime} \times 3.5$, with north up and east to the left. The X-ray position uncertainties are indicated by the circles, and the proposed optical counterparts are shown by the plus signs. [See the electronic edition of the Journal for a color version of this figure.]

$\mathrm{X}$-ray sources that are somewhat fainter than the majority of the sources in Table 2. This faintness, together with the hardness of the X-ray spectrum, likely reflects extragalactic origins of the sources (i.e., they are active galaxies); in Figure 6 these sources are largely those with the highest X-ray-to-IR flux ratios, which are the most similar to those of the extragalactic sample.
Overall, 1RXS J205812.8+292037 is consistent with having an association with one of the identified 2MASS sources. A Chandra follow-up observation would have made the case secure, but it was not selected for Chandra due to an oversight. As with 1RXS $\mathrm{J} 205042.9+284643$, we do not believe that 1RXS J205812.8+ 292037 is a neutron star, but we cannot rule out this possibility. 

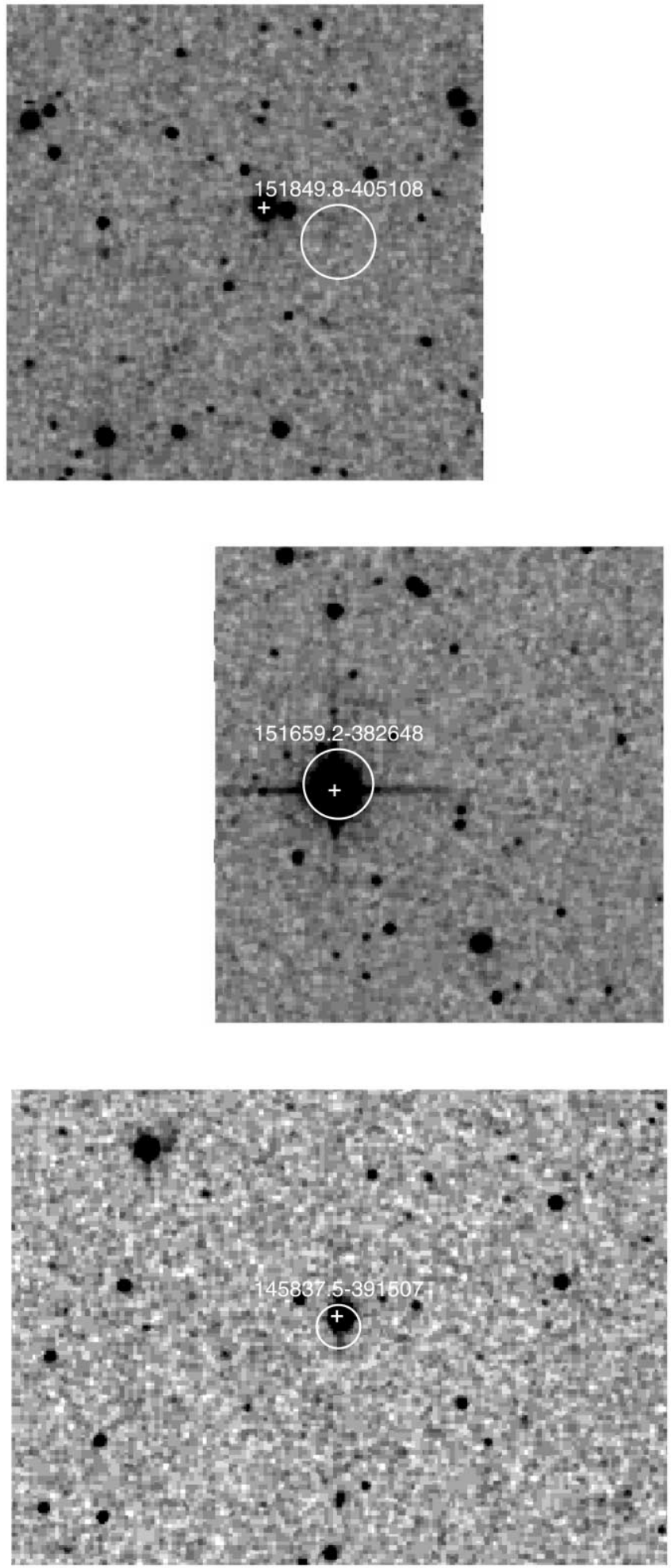

FIG. 16. - Additional 2MASS $K_{s}$-band images of the sources in SNR G330.0+15.0 from Table 2. [See the electronic edition of the Journal for a color version of this figure.]

\subsection{4. $1 R X S J 150139.6-403815$}

The BSC lists 1RXS J150139.6-403815 as having $0.12 \pm$ 0.02 counts $\mathrm{s}^{-1}$ in the PSPC, with hardness ratios of HR $1=$ $0.88 \pm 0.11$ and HR2 $=0.14 \pm 0.20$. The Chandra source is fainter than that of 1RXS J193458.1+335301, but nonetheless it appears extended, as shown in Figure 27. Since this source is
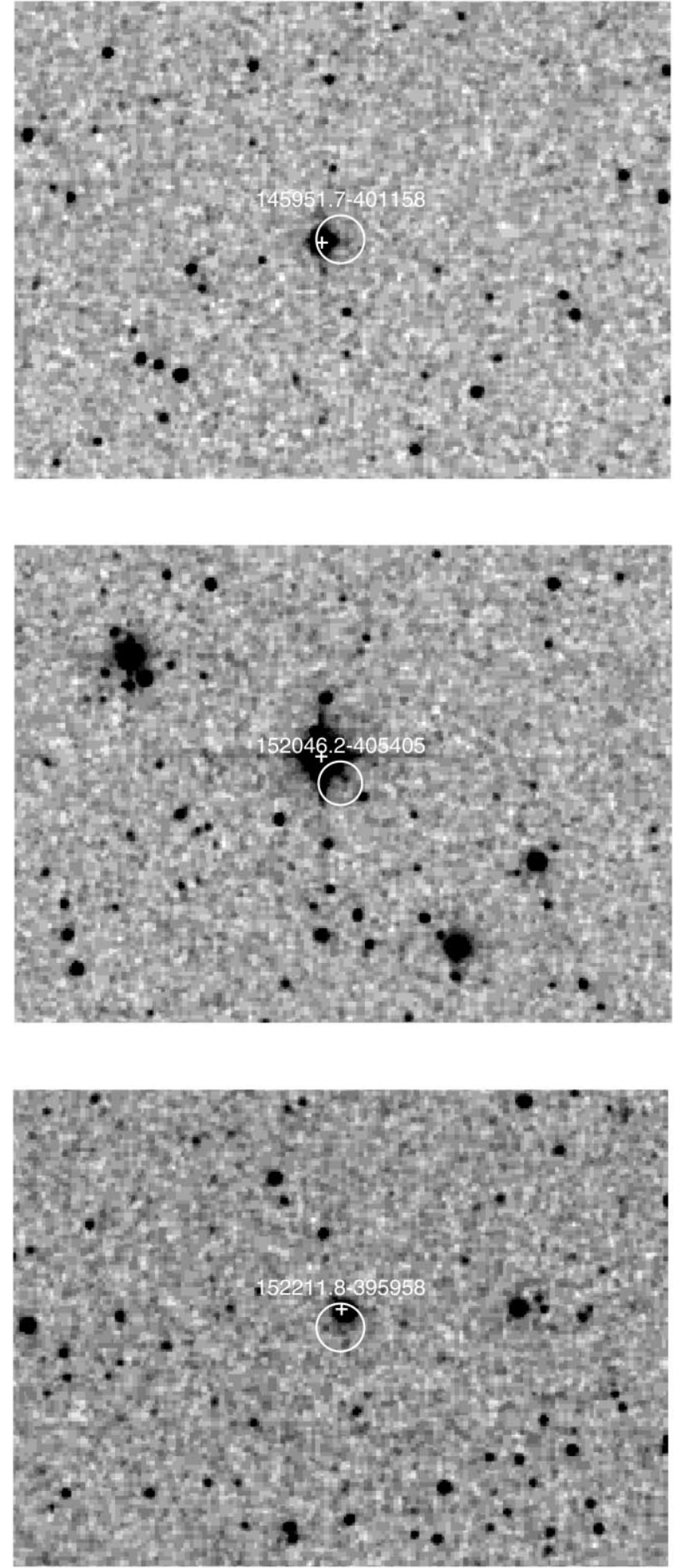

more diffuse than 1RXS J193458.1+335301, the spatial measurements are not as precise, but the center is at approximately $15^{\mathrm{h}} 01^{\mathrm{m}} 41^{\mathrm{s}} \mathrm{s},-40^{\circ} 38^{\prime} 08^{\prime \prime}$. The total extent of the source is $\approx 1^{\prime}$ in radius. As with 1RXS J193458.1+335301, while there is some offset between the ROSAT and Chandra positions, this does not appear inconsistent with the position uncertainties for such extended sources. Again, we can be quite confident that the Chandra 

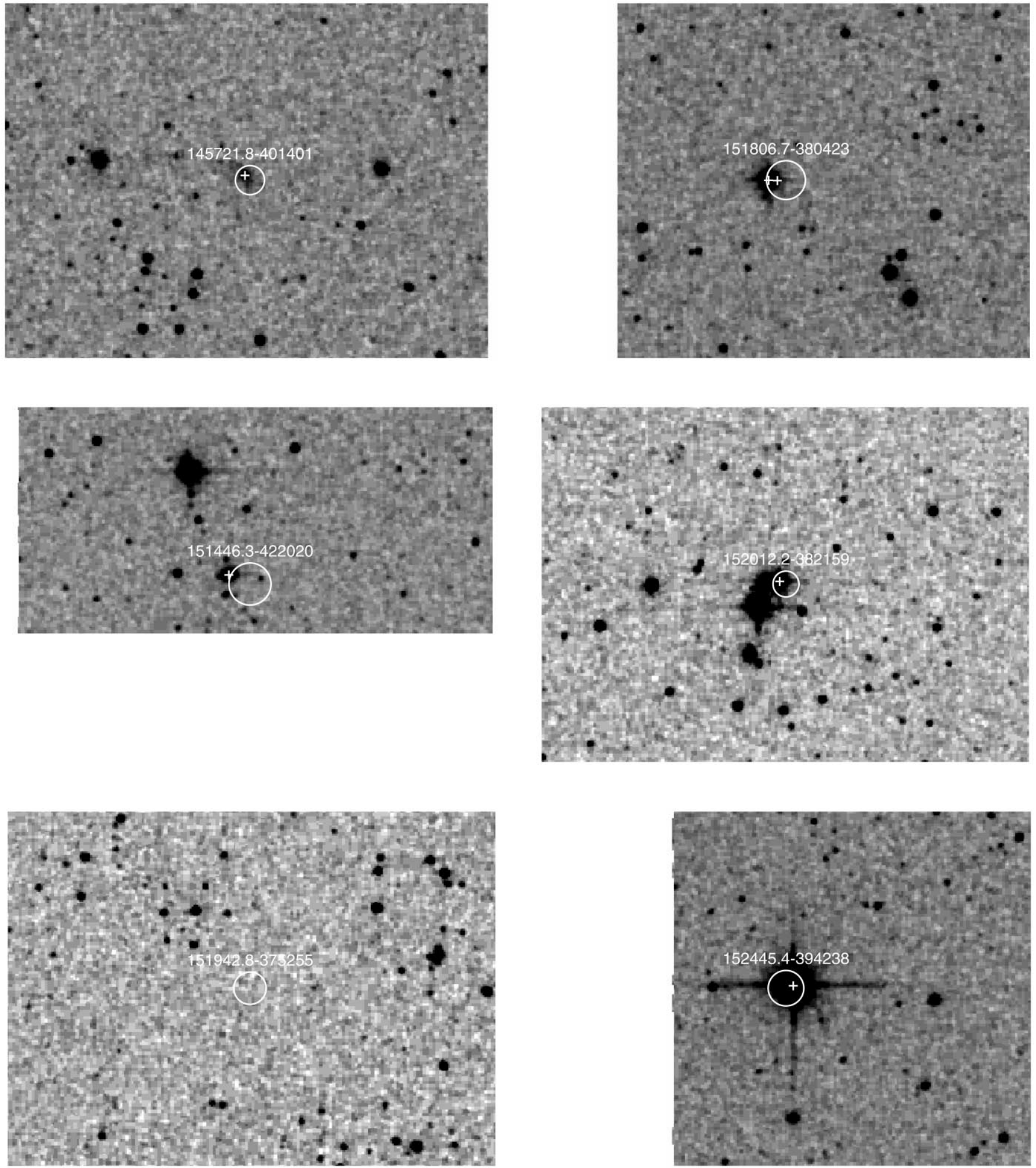

FIG. 17. - Additional 2MASS $K_{\mathrm{s}}$-band images of the sources in SNR G330.0+15.0 from Table 2. [See the electronic edition of the Journal for a color version of this figure.]

source is 1RXS J150139.6-403815, since there are no other sources nearby.

Similar to our analysis of 1RXS J193458.1+335301 (§ 5.3.1), we extracted photon events from a $112^{\prime \prime} \times 90^{\prime \prime}$ region and created source and background response files using the CIAO task acisspec. We then fit the data in Sherpa, where the events were binned so that each bin had $\geq 25$ counts.
There are 1305 source counts and 478.5 background counts. The data are well fit (Fig. 28) by an absorbed power-law model, with $N_{\mathrm{H}}=(1.0 \pm 0.4) \times 10^{21} \mathrm{~cm}^{-2}, \Gamma=1.65 \pm 0.15$, and an amplitude of $(4.7 \pm 0.6) \times 10^{-4}$ photons $\mathrm{cm}^{-2} \mathrm{~s}^{-1} \mathrm{keV}^{-1}$ at $1 \mathrm{keV}$ (giving $\chi^{2}=21.6$ for 32 degrees of freedom; all uncertainties are $1 \sigma$ ). The observed flux from this model is $2.6 \times$ $10^{-12} \mathrm{ergs} \mathrm{cm}^{-2} \mathrm{~s}^{-1}(0.3-8.0 \mathrm{keV})$, and the unabsorbed flux is 


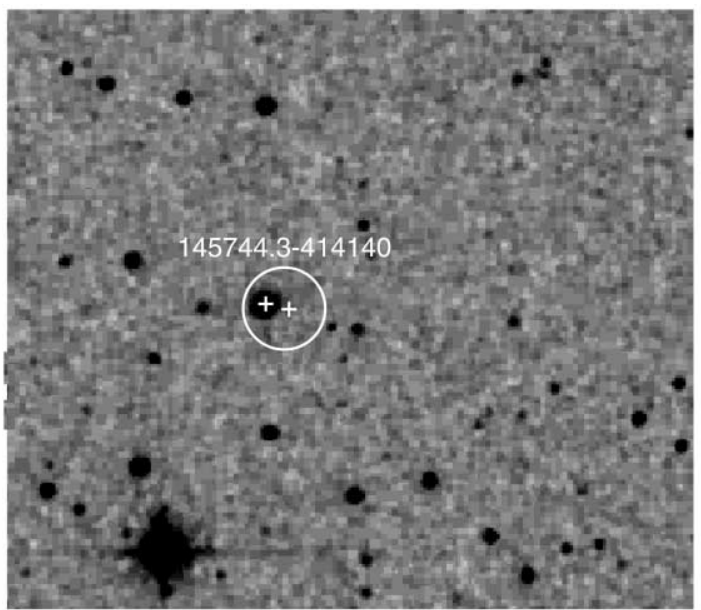

FIG. 18.-Additional 2MASS $K_{s}$-band image of the sources in SNR G330.0+ 15.0 from Table 2. [See the electronic edition of the Journal for a color version of this figure.]

$3.0 \times 10^{-12} \mathrm{ergs} \mathrm{cm}^{-2} \mathrm{~s}^{-1}(0.3-8.0 \mathrm{keV})$. The column density is higher than but consistent (given the uncertainties) with both the column density of SNR G330.0+15.0 and the total expected along this line of sight $\left(6 \times 10^{20} \mathrm{~cm}^{-2}\right)$.

As with 1RXS J193458.1+335301, we considered different models for 1RXS J150139.6-403815. Figure 29 does not identify a single hot source, so a thermal Galactic nebula is unlikely. Our first idea was that 1RXS J150139.6-403815 is a PWN. The size is about right and the spectrum is typical for PWNe. However, as with 1RXS J193458.1+335301 there is a problem: 1RXS J150139.6-403815 is outside of SNR G156.2+5.7, and the largely symmetric morphology rules out an association between 1RXS J150139.6-403815 and SNR G330.0+15.0 (i.e., 1RXS J150139.6-403815 cannot be a bow shock nebula). 1RXS J150139.6-403815 could instead be a pressure-confined bubble PWN related to another supernova; we searched the Sydney University Molongolo Sky Survey (SUMSS; Bock et al. 1999) for evidence of radio emission from or another supernova shell surrounding 1RXS J150139.6-403815, but there is no extended or pointlike emission present at the position of 1RXS J150139.6-403815 nor is there any sign of a new SNR around it. SUMSS is particularly sensitive to extended emission and would almost certainly have identified any SNR around 1RXS J150139.6-403815. As with 1RXS J193458.1+335301, the lack of a clear SNR shell does not mean that 1RXS J150139.6403815 is not a PWN.

We then examined possible extragalactic classifications for 1RXS J150139.6-403815. This source is larger than 1RXS $\mathrm{J} 193458.1+335301$ and is more compatible with the sizes of typical galaxy clusters $\left(\gtrsim 30^{\prime \prime}\right)$ : a fit to a $\beta$-model has $r_{c}=32^{\prime \prime}$ and $\beta=0.4$. The spectral data are reasonably well fit by a Raymond $\&$ Smith (1977) plasma model, having $N_{\mathrm{H}}=(5 \pm 2) \times 10^{20} \mathrm{~cm}^{-2}$, $k T=9_{-2}^{+5} \mathrm{keV}$, and a normalization ${ }^{11}$ of $(5.6 \pm 0.5) \times 10^{-3} \mathrm{~cm}$ (giving $\chi^{2}=22.0$ for 32 degrees of freedom), such as what one would expect for a cluster (White et al. 1997). With this model the observed flux is $2.7 \times 10^{-12} \mathrm{ergs} \mathrm{cm}^{-2} \mathrm{~s}^{-1}(0.3-8.0 \mathrm{keV})$, and the unabsorbed flux is $2.9 \times 10^{-12} \mathrm{ergs} \mathrm{cm}^{-2} \mathrm{~s}^{-1}$.

Examining the 2MASS image we see an extended elliptical source, 2MASX J15014110-4038093, near the center of the $\mathrm{X}$-ray emission (Fig. 29). This source has a radius of $\approx 10^{\prime \prime}(20 \mathrm{mag}$ $\operatorname{arcsec}^{-2}$ isophotal radius), a $K_{s}$ magnitude of 12.7 mag within that radius, and $J-K_{S}=1.2 \mathrm{mag}$. Higher resolution optical images of 1RXS J150139.6-403815 (Fig. 29) show that 2MASX J15014110-4038093 is partially decomposed into two sources: an extended source labeled A that is at the exact position of 2MASX J15014110-4038093 (within uncertainties), and a source labeled B $3^{\prime \prime}$ to the east. There is also another extended source labeled $\mathrm{C} 5^{\prime \prime}$ to the northeast, but this is a separate 2MASS source (2MASS J15014145-4038068). We performed a rough photometric calibration using 80 stars from the USNO-B1.0 catalog ${ }^{12}$ and then ran SExtractor (Bertin \& Arnouts 1996) on the images; the results for sources A-C are given in Table 6. Source A is very clearly extended, although it is not as large as 2 MASX

11 The normalization follows the XSPEC units of $10^{-14}\left\{4 \pi\left[D_{A}(1+z)\right]^{2}\right\}^{-1}$ $\times \int d V n_{e} n_{\mathrm{H}}$, where $D_{A}$ is the angular-size distance (in $\left.\mathrm{cm}\right), n_{e}$ is the electron density (in $\mathrm{cm}^{-3}$ ), and $n_{\mathrm{H}}$ is the hydrogen density (in $\mathrm{cm}^{-3}$ ).

12 This calibration agreed with the nominal calibration at http://occult.mit .edu/instrumentation/magic.

TABLE 4

Log of Chandra ACIS Observations

\begin{tabular}{|c|c|c|c|}
\hline Source & Date & $\begin{array}{l}\text { Exposure } \\
(\mathrm{ks})\end{array}$ & Subarray Mode \\
\hline \multicolumn{4}{|l|}{ SNR G65.3+5.7: } \\
\hline 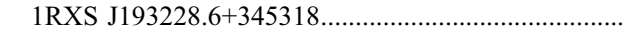 & 2002 Dec 08 (obs/3887) & 3.7 & $1 / 4$ \\
\hline 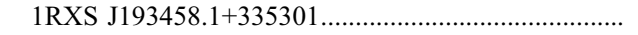 & 2003 Jan 26 (obs/3888) & 3.5 & $1 / 4$ \\
\hline \multicolumn{4}{|l|}{ SNR G74.0-8.5: } \\
\hline 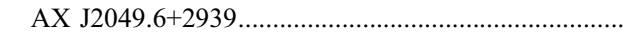 & 2003 Mar 19 (obs/3889) & 3.2 & $1 / 2$ \\
\hline 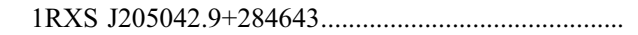 & 2003 Mar 19 (obs/3890) & 3.9 & $1 / 4$ \\
\hline \multicolumn{4}{|l|}{ SNR G160.9+2.6: } \\
\hline 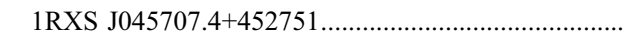 & 2003 Jan 04 (obs/3878) & 5.2 & $1 / 4$ \\
\hline \multicolumn{4}{|l|}{ SNR G205.5+0.5: } \\
\hline 1RXS J050339.8+451715 & 2003 Jan 08 (obs/3879) & 5.4 & $1 / 4$ \\
\hline 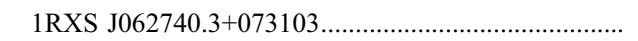 & 2003 Mar $11(\mathrm{obs} / 3880)$ & 3.4 & $1 / 4$ \\
\hline 1E $0627.4+0537$ & 2002 Dec 07 (obs/3881) & 3.5 & $1 / 2$ \\
\hline 1E $0630.9+0611 \ldots \ldots \ldots$ & 2003 Apr 22 (obs/3882) & 3.6 & $1 / 2$ \\
\hline 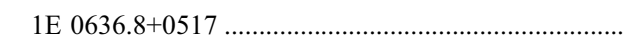 & 2003 Mar $11(\mathrm{obs} / 3883)$ & 3.8 & $1 / 2$ \\
\hline \multicolumn{4}{|l|}{ SNR G330.0+15.0: } \\
\hline 1RXS J150139.6-403815 & 2003 Mar 18 (obs/3884) & 2.9 & $1 / 4$ \\
\hline 1RXS J150818.8-401730 & $2003 \mathrm{Mar} 18$ (obs/3885) & 3.7 & $1 / 4$ \\
\hline 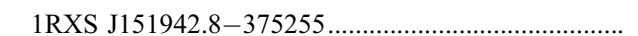 & 2003 Mar $10(\mathrm{obs} / 3886)$ & 2.9 & $1 / 4$ \\
\hline
\end{tabular}



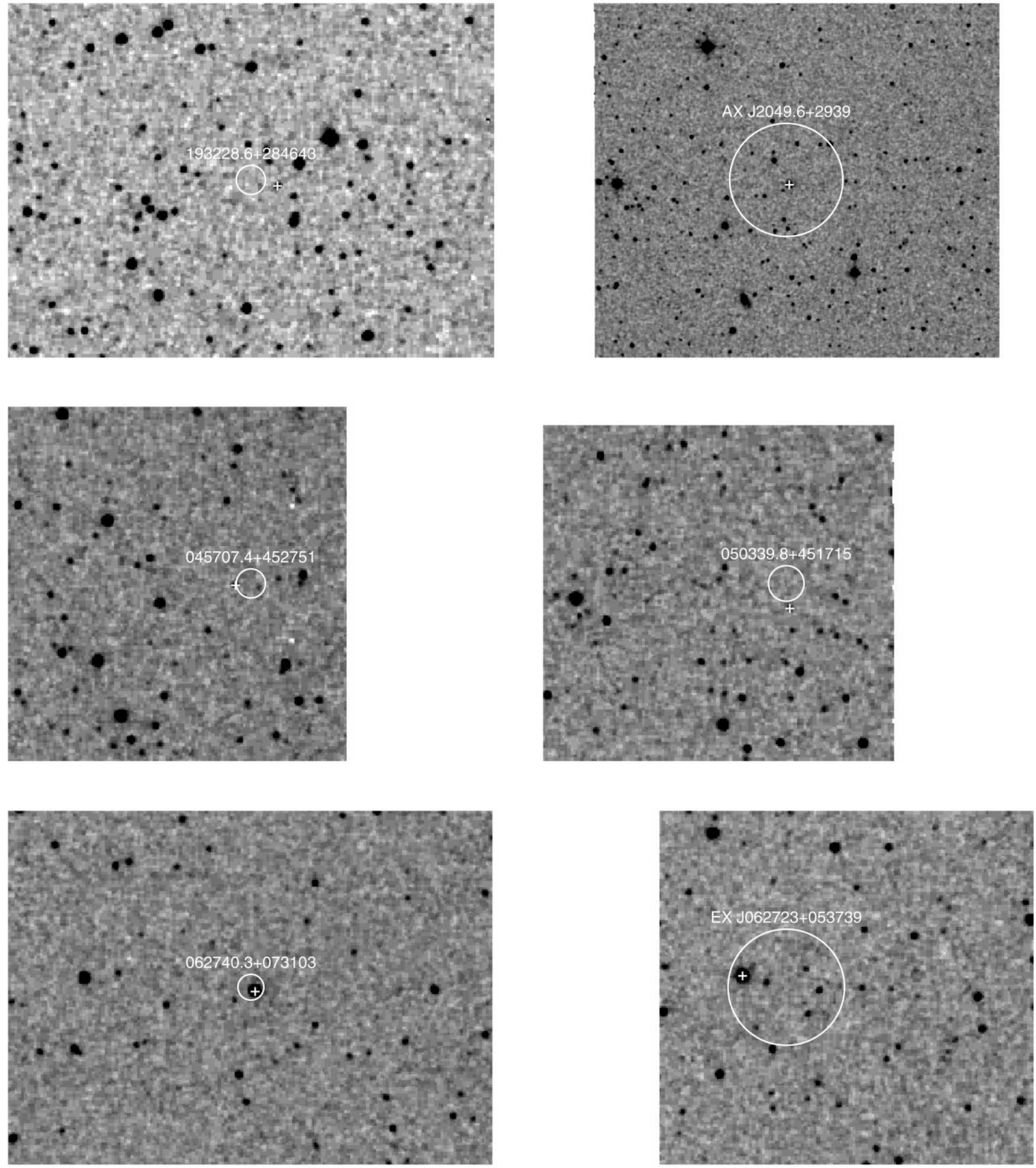

FIG. 19.-2MASS $K_{\mathrm{s}}$-band images of the sources from Table 4 with pointlike X-ray sources and secure counterpart identifications. The images are $5^{\prime} \times 3.5$ (except for that of AX J2049.6+2939, which is $10^{\prime} \times 7^{\prime}$ ), with north up and east to the left. The BSC/ASCA/Einstein X-ray position uncertainties are indicated by the circles, and the Chandra positions and optical counterparts are shown by the plus signs. [See the electronic edition of the Journal for a color version of this figure.]

J15014110-4038093 (the FWHM of the IR emission is $\approx 6^{\prime \prime}$ ). Source B is very likely unresolved (within uncertainties), and source $\mathrm{C}$ is extended. Source $\mathrm{A}$ is very red $(B-R \approx 2.6 \mathrm{mag})$, consistent with the 2MASS data. We believe that the 2MASS source is primarily due to source $\mathrm{A}$, given the position coincidence and the extreme redness of $\mathrm{A}$ compared to $\mathrm{B}$ or $\mathrm{C}$. If this is the case, however, then $\mathrm{A}$ has the relatively blue color of $R-K_{s} \approx$
$-3.2 \mathrm{mag}$, but this could be partly due to the difficulties of measuring an extended source from images with drastically different seeing (2MASS vs. MagIC $R$ band).

The IR colors of 2MASX J15014110-4038093 are similar to those of the brighter galaxies in known clusters (e.g., Kodama \& Bower 2003). Therefore, 2MASX J15014110-4038093 could be the central galaxy of an unknown cluster. The X-ray temperature 

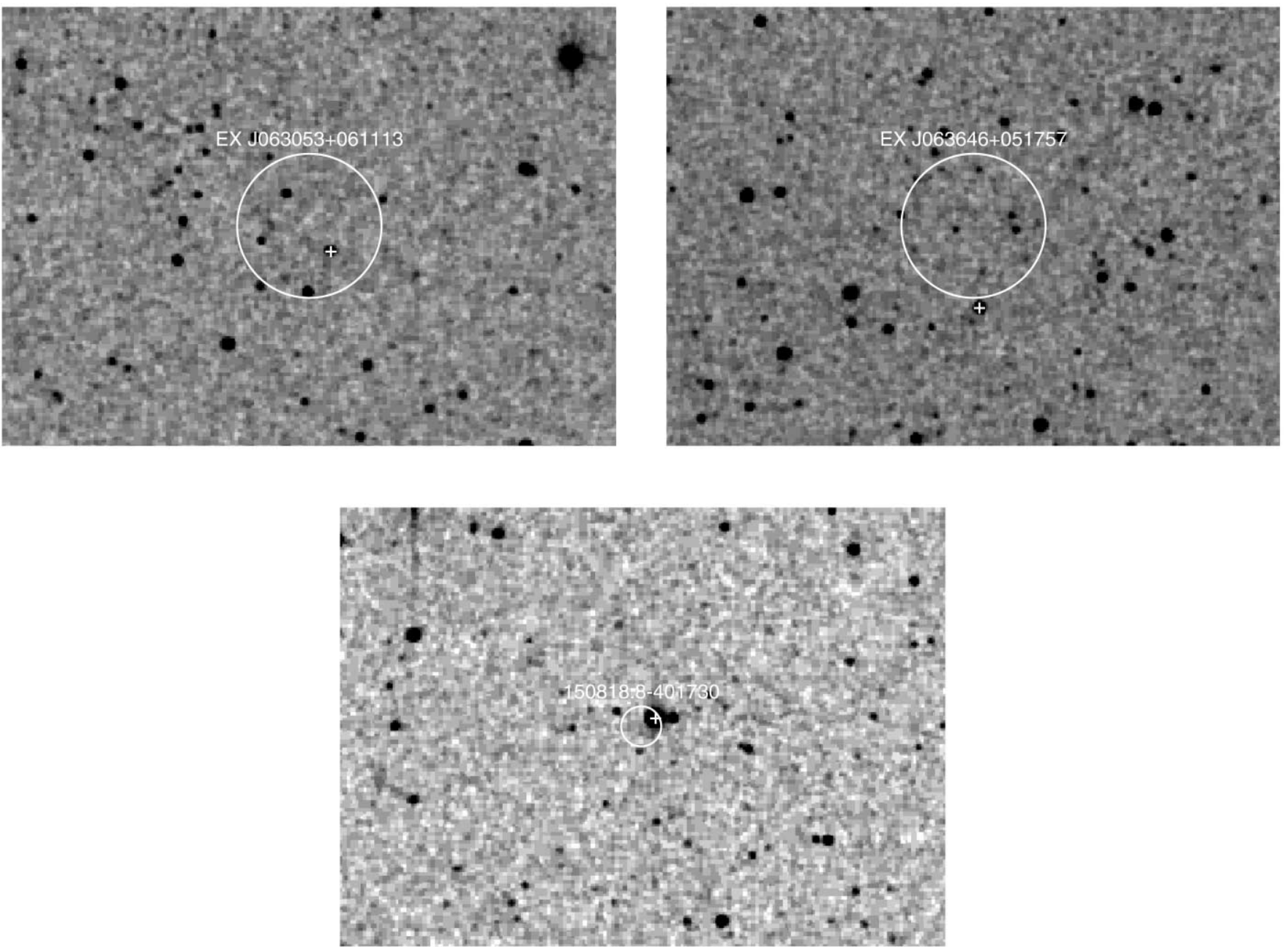

FIG. 20.-Additional 2MASS $K_{\mathrm{s}}$-band images of the sources from Table 4 with pointlike X-ray sources and secure counterpart identifications. [See the electronic edition of the Journal for a color version of this figure.]

is reasonably high, implying a high luminosity $\left(\sim 10^{45} \mathrm{ergs} \mathrm{s}^{-1}\right.$; Mushotzky 2004), so this source cannot be part of a nearby, low- $L$ group. However, the value of $\beta$ is lower than the values of most known clusters (Vikhlinin et al. 1999a) and is more similar to those of low- $L$ systems (Mulchaey et al. 2003).

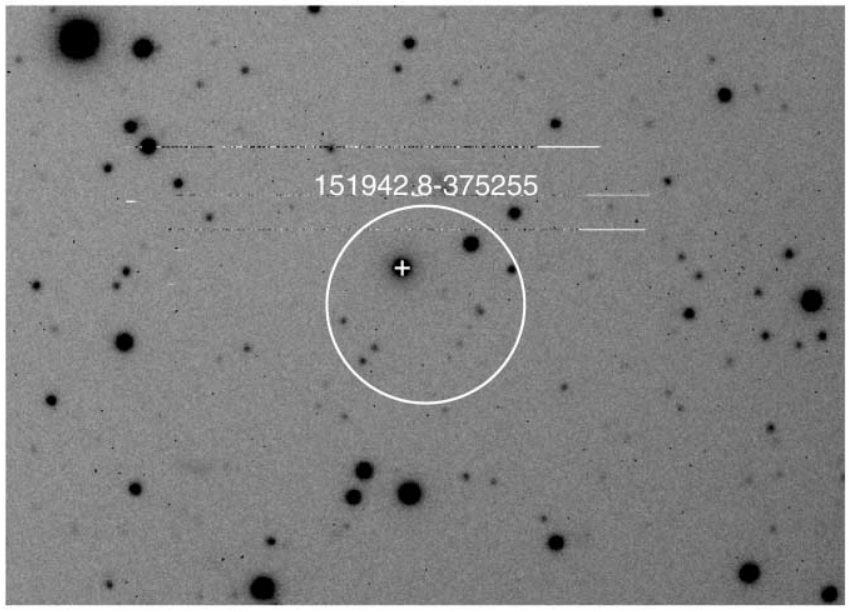

While 1RXS J150139.6-403815 is compatible with the sizes and spectra of early-type galaxies and there is an extended optical/IR source near the peak of the X-ray emission, the scenario is not entirely consistent. The optical/IR source is, like those in 1RXS J193458.1+335301, about 7 mag fainter than expected

FIg. 21.-Magellan images of 1RXS J151942.8-375255. Left: MagIC $R$-band image. Right: PANIC $K_{\mathrm{s}}$-band image. The images are $\approx 45^{\prime \prime} \times 30^{\prime \prime}$, with north up and east to the left. The BSC X-ray position uncertainties are indicated by the circles, and the Chandra positions and optical counterparts are shown by the plus signs. [See the electronic edition of the Journal for a color version of this figure.]

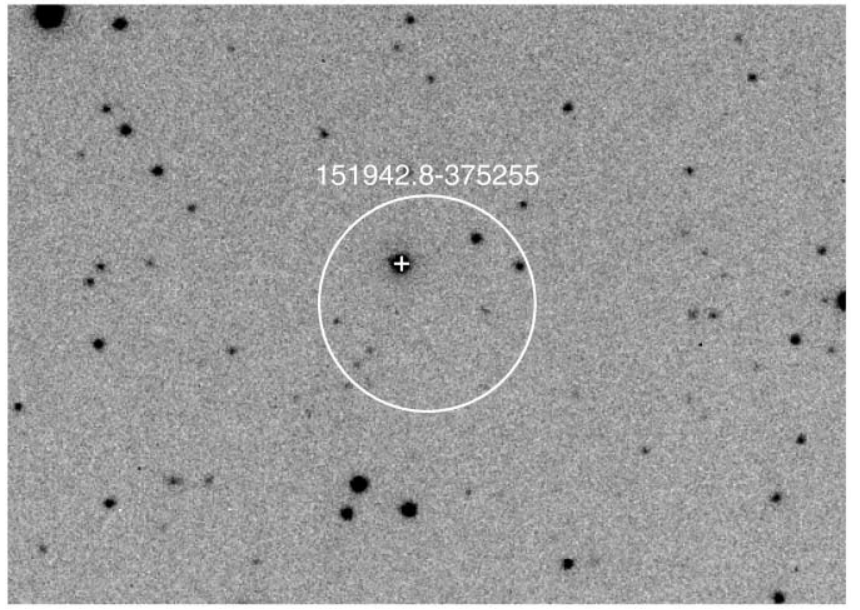


TABLE 5

LOG OF OptICAL/IR ObSERVATIONS

\begin{tabular}{|c|c|c|c|c|c|}
\hline Source & Date & Telescope & Instrument & Band(s) & $\begin{array}{c}\text { Exposure } \\
\text { (s) }\end{array}$ \\
\hline 1RXS J193458.1+335301..................... & $2003 \mathrm{Jul} 03$ & P200 & LFC & $g^{\prime}$ & 300 \\
\hline 1RXS J193458.1+335301_............... & 2003 Jul 24 & $\mathrm{P} 60$ & P60CCD & $\mathrm{H} \alpha / \mathrm{Off}-\mathrm{b} a n d$ & 1200 \\
\hline \multirow{2}{*}{ 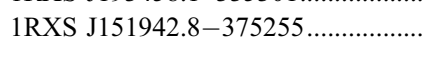 } & 2003 Apr 04 & Magellan II/Clay & MagIC & $R$ & 930 \\
\hline & 2003 Apr 18 & Magellan II/Clay & PANIC & $K_{s}$ & 200 \\
\hline \multirow[t]{2}{*}{ 1RXS J150139.6-403815 ….............. } & 2004 Feb 16 & Magellan II/Clay & MagIC & $R$ & 60 \\
\hline & & & & $B$ & 160 \\
\hline
\end{tabular}

Notes.-The telescopes and instruments used were as follows: LFC, the Large Format Camera on the Palomar 200 inch; P60CCD, the CCD camera on the Palomar 60 inch; MagIC, the Raymond and Beverly Sackler Magellan Instant Camera on the $6.5 \mathrm{~m}$ Clay (Magellan II) telescope; PANIC, Persson's Auxiliary Nasmyth Infrared Camera on the $6.5 \mathrm{~m}$ Clay (Magellan II) telescope (Martini et al. 2004).

a Observed by C. Rakowski.

(the predicted magnitude following eq. [1] is $K_{s} \approx 5 \mathrm{mag}$ ). This is far greater than the variation seen among galaxies. We do not believe that the difference can be due to an excess of soft emission in 1RXS J193458.1+335301 or 1RXS J150139.6-403815 (eq. [1] refers only to the contribution of hard point sources), as the spectra of 1RXS J193458.1+335301 and 1RXS J150139.6403815 are hard and similar to the prototypical sources assumed in Kim \& Fabbiano (2004), and when one fits primarily for the soft emission (as in Brown \& Bregman 1998), one finds a relation similar to that of Kim \& Fabbiano (2004). It is possible that 1RXS J150139.6-403815 is an overluminous elliptical galaxy, such as those discussed in Vikhlinin et al. (1999b), as the size, optical/X-ray flux ratio, and luminosity are similar to these sources $\left(L_{\mathrm{X}} / L_{\mathrm{opt}} \sim 10^{32} \mathrm{ergs} \mathrm{s}^{-1} L_{\odot}^{-1} ; L_{\mathrm{opt}} \sim 10^{11} L_{\odot}\right.$ assuming $\left.z \sim 0.1\right)$, but again there are difficulties: the temperature of 1RXS J150139.6403815 is considerably higher than those of Vikhlinin et al. (1999b), and the value of $\beta$ is too low.

We see that no scenario is entirely consistent for $1 \mathrm{RXS}$ J150139.6-403815. PWNe, isolated elliptical galaxies, and galaxy clusters all have problems. We believe it likely that $1 \mathrm{RXS}$ J150139.6-403815 does have an extragalactic origin, as 2MASX

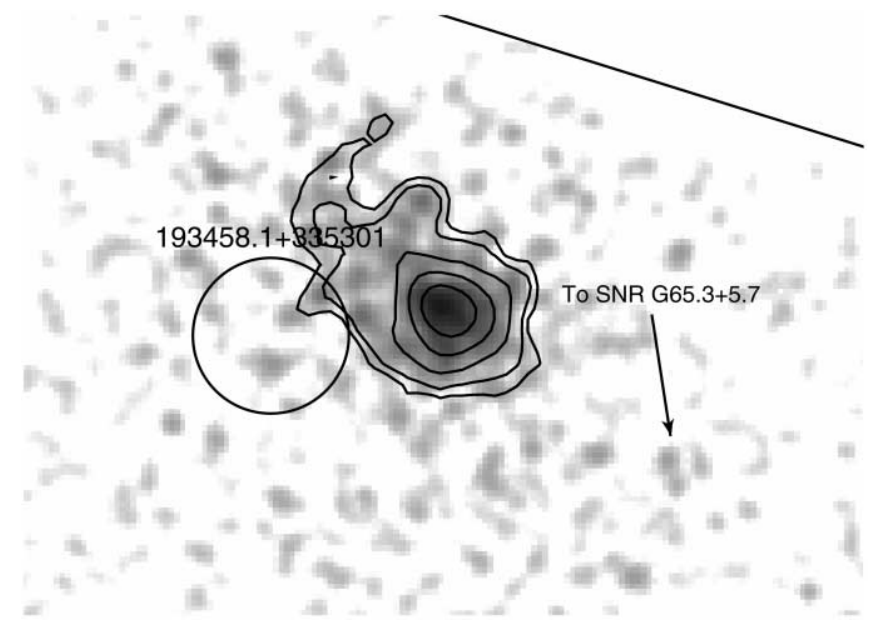

FIG. 22.-Chandra ACIS-S3 image of 1RXS J193458.1+335301. The BSC source and uncertainty are shown by the circle with radius $14^{\prime \prime}$. The gray scale is proportional to the logarithm of the counts in 2 pixel bins, and the image has been smoothed with a Gaussian filter with a radius of 3 pixels. The contours are in steps from 0.2 to 2.2 counts per bin, with spacing proportional to the square root of the counts. The arrow indicates the direction to the center of SNR G65.3+5.7. The box shows the approximate extent of the ACIS subarray. The image is $\approx 150^{\prime \prime} \times 110^{\prime \prime}$, with north up and east to the left.
J15014110-4038093 looks like an elliptical galaxy and it is probably associated with the X-ray emission: there are 39 extended 2MASS sources within $20^{\prime}$ of 1RXS J150139.6-403815, giving a false-association rate of $0.005 \%$ for a source within $1 . " 3$. However, it is not clear exactly what 1RXS J150139.6-403815 is. As with 1RXS J193458.1+335301, deeper X-ray observations and optical spectroscopy should be conclusive for 1RXS J150139.6-403815.

\section{DISCUSSION AND CONCLUSIONS}

We have fully investigated the population of ROSAT BSC point sources in six large-diameter SNRs. Our identifications of counterparts to 50 of the 54 sources are quite secure; in most cases the positional coincidence between the X-ray and optical/IR sources has been augmented by identification of an abnormal stellar type (variable, $\mathrm{T}$ Tauri, binary, etc.), by the extreme brightness (and hence rarity) of the optical source, or by a previous classification in the literature. This conclusion echoes that of Rutledge et al. (2003), who searched for older neutron stars using ROSAT and found only previously identified neutron stars, along with 17 sources that are definitely not neutron stars and 13 that are probably not.

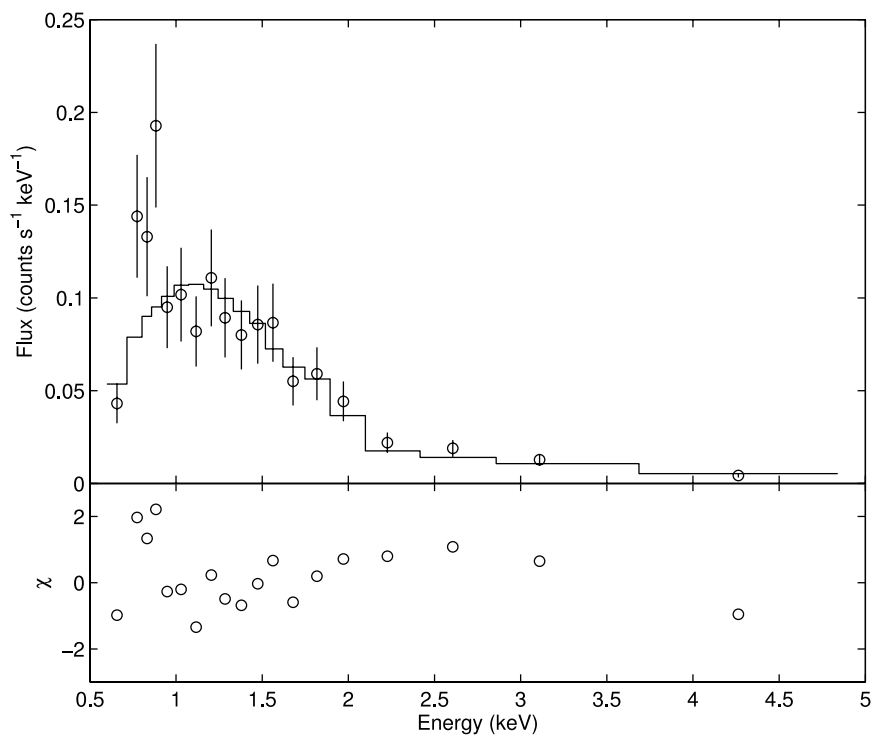

FIG. 23.-Chandra ACIS-S3 spectrum of 1RXS J193458.1+335301, with the best-fit power-law model $\left[N_{\mathrm{H}}=(3.0 \pm 0.6) \times 10^{21} \mathrm{~cm}^{-2}\right.$, photon index $\Gamma=2.4 \pm 0.2$, and an amplitude of $(4.6 \pm 0.8) \times 10^{-4}$ photons $\mathrm{cm}^{-2} \mathrm{~s}^{-1}$ $\mathrm{keV}^{-1}$ at $1 \mathrm{keV}$ ]. The residuals are plotted in the bottom panel. 


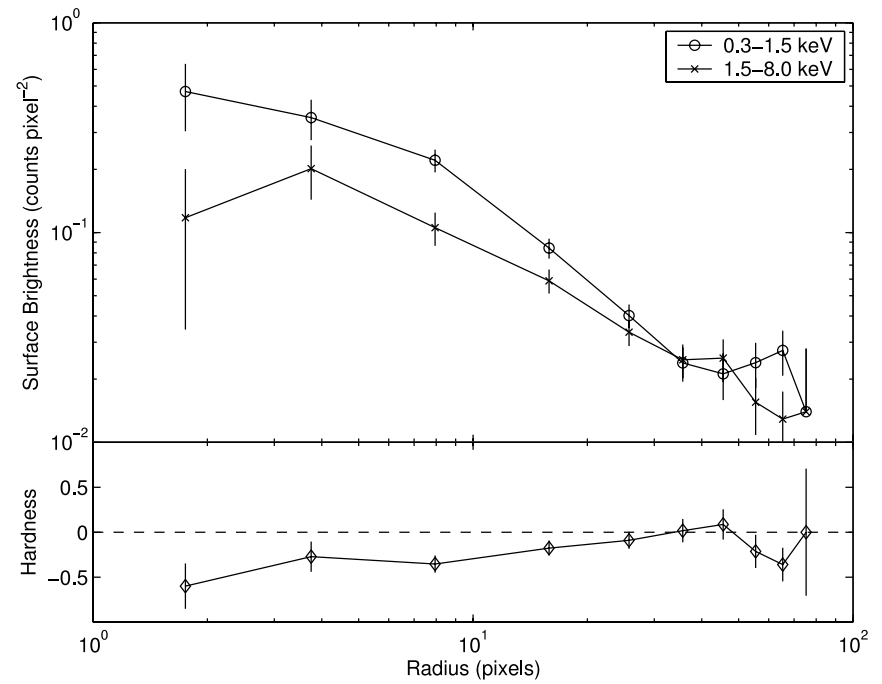

FIG. 24.-Top: Radial profiles of the events for 1RXS J193458.1+335301 in two different energy bands. The background-subtracted surface brightness is plotted against radius for soft $(0.3-1.5 \mathrm{keV})$ and hard $(1.5-8.0 \mathrm{keV})$ bands. Bottom: Hardness of the radial profile, defined as $\left(B_{H}-B_{S}\right) /\left(B_{H}+B_{S}\right)$, where $B_{H}\left(B_{S}\right)$ is the surface brightness in the $1.5-8.0 \mathrm{keV}(0.3-1.5 \mathrm{keV})$ band. There appears to be a slight excess of soft photons toward the center.

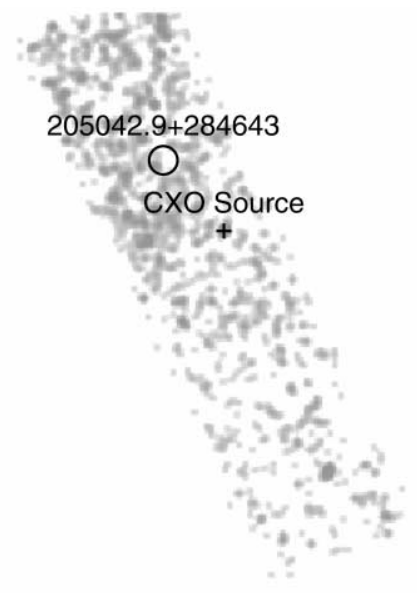

FIG. 26.-Chandra ACIS-S image of the field around 1RXS J205042.9+ 284643. The position of the ROSAT source is shown by the circle with a radius of $12^{\prime \prime}$, which is the $1 \sigma$ position uncertainty. The only significant point source detected in the Chandra observation is shown by the plus sign, $79^{\prime \prime}$ away from the ROSAT position; see $\S$ 5.3.2. The gray scale is proportional to the logarithm of the $0.3-5.0 \mathrm{keV}$ counts in 4 pixel bins, and the image has been smoothed with a Gaussian filter with a radius of 3 pixels.
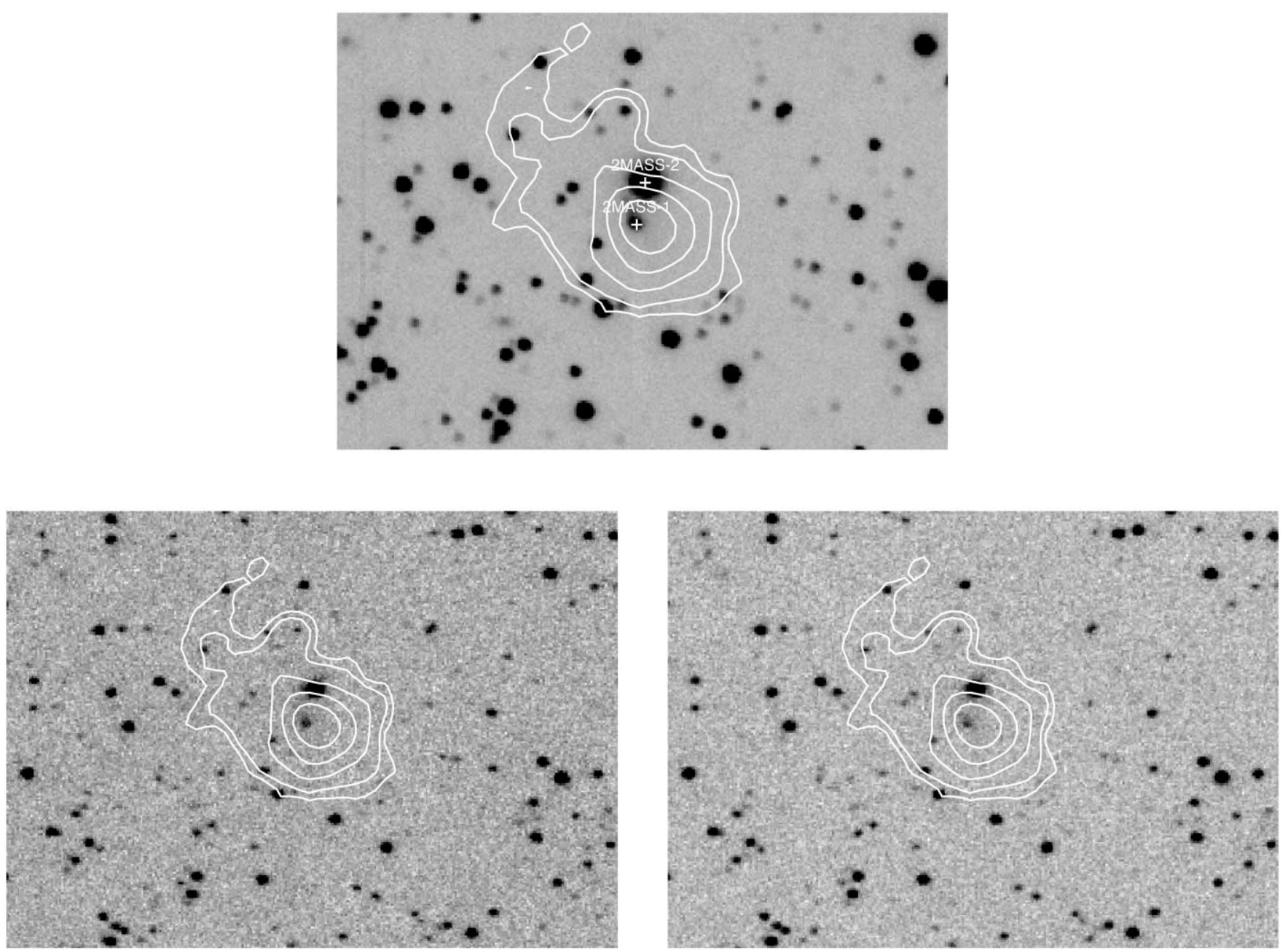

FIG. 25.-Top: Palomar 200 inch $g^{\prime}$-band image of 1RXS J193458.1+335301. Bottom: Palomar 60 inch images of 1RXS J193458.1+335301, taken with H $\alpha$ (left) and off-band $\left(\right.$ right $; 6584 \AA$ ) filters. The images are $\approx 110^{\prime \prime} \times 80^{\prime \prime}$, with north up and east to the left. The contours are those from Fig. 22 showing the extent of the $\mathrm{X}$-ray emission. The two 2MASS sources identified near the peak of the X-ray emission are indicated with plus signs in the top image; the southern source is $2 \mathrm{MASS}$ $\mathrm{J} 19345569+3353063$, while the northern one is 2MASS J19345557+3353136. We do not detect any diffuse $\mathrm{H} \alpha$ emission associated with 1 RXS J193458.1+335301. 


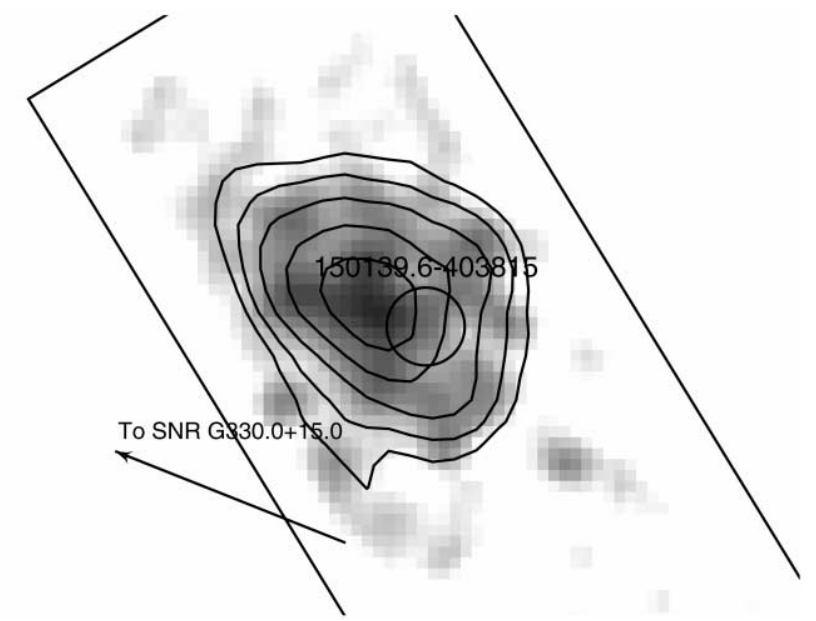

FIG. 27.-Chandra ACIS-S3 image of 1RXS J150139.6-403815. The BSC source and uncertainty are shown by the circle with radius $14^{\prime \prime}$. The gray scale is proportional to the logarithm of the counts in 8 pixel bins, and the image has been smoothed with a Gaussian filter with a radius of 3 pixels. The contours are in steps from 1.5-3.8 counts per bin, with spacing proportional to the square root of the counts. The box shows the approximate extent of the ACIS subarray. The image is $\approx 300^{\prime \prime} \times 210^{\prime \prime}$, with north up and east to the left.

The remaining sources, as discussed in $\S 5.3$, are more intriguing. However, none of them is likely to be a neutron star associated with one of the SNRs in Table 1. To begin with, all are outside their SNRs. While this is not impossible for older sources and high-velocity neutron stars (e.g., Gaensler \& Johnston 1995), it lessens the chance of association.

For 1RXS J193458.1+335301 and 1RXS J150139.6-403815, the X-ray morphologies rule out associations, since any PWNe outside the SNRs would likely have elongated bow shock appearances, in contrast to what we see (of course, 1RXS J193458.1+ 335301 and/or 1RXS J150139.6-403815 could be extragalactic). 1RXS J205042.9+284643 and 1RXS J205812.8+292037, neither of which has a Chandra detection, are more uncertain. 1RXS $\mathrm{J} 205042.9+284643$ is likely a flare star. 1RXS J205812.8+292037 does not have a provisional classification but is probably extragalactic in origin.

Since we have ruled out (to some degree of certainty) neutron stars in all six SNRs considered here, we can follow Paper I and draw the X-ray luminosity limits on a cooling diagram. This is shown in Figure 30. To account for the uncertainties of 1RXS J205042.9+284643 and 1RXS J205812.8+292037, both in SNR G74.0-8.5, we have adjusted the luminosity of that SNR from Table 1 to correspond to 0.15 counts $\mathrm{s}^{-1}$, which is above the count rates of both of the uncertain sources and therefore a more secure limit. Further X-ray observations of 1RXS J205812.8+ 292037 would very likely detect counterparts (for 1RXS J205042.9+ 284643, it might have only been included in the BSC due to a flare, and therefore significantly deeper X-ray observations may be necessary). With secure counterparts, the limit for SNR G74.08.5 would decrease by a factor of 3 .

The limits in Figure 30 are not as uniform or as constraining as those from Paper I. The lack of uniformity is due to the sample construction: the different distances and column densities of the SNRs make the BSC limit of 0.05 counts $\mathrm{s}^{-1}$ translate into different luminosities. So, SNRs G74.0-8.5, G330.0+15.0, and G65.3+ 5.7 all have reasonably tight limits (and those of SNR G74.0-8.5 could get better). SNRs G160.9+2.6 and G205.5+0.5 have loose limits primarily due to uncertain distances; we have used the up-

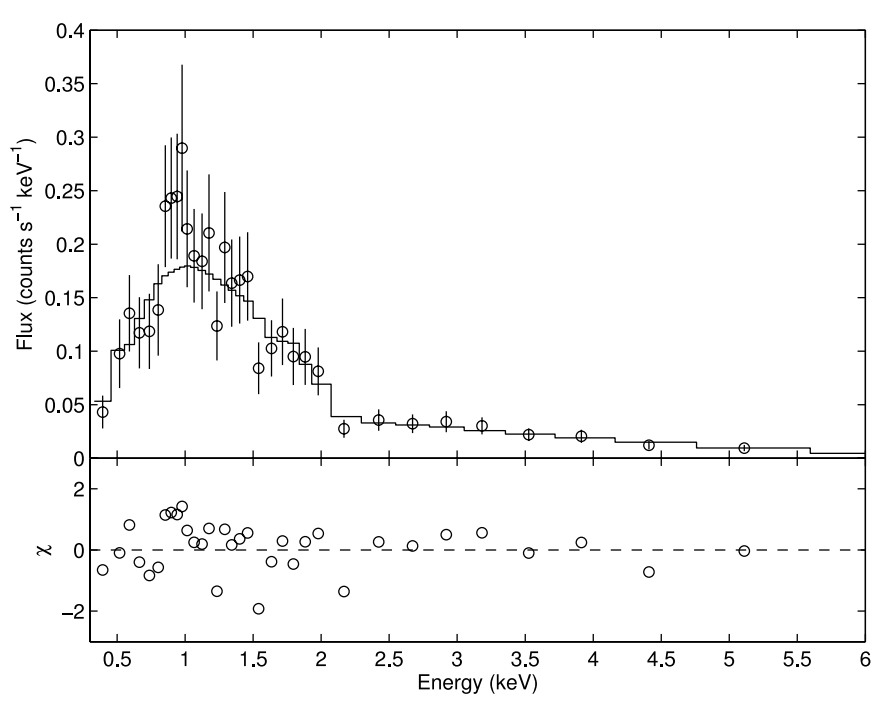

FIG. 28.-Chandra ACIS-S3 spectrum of 1RXS J150139.6-403815, with the best-fit power-law model $\left[N_{\mathrm{H}}=(1.0 \pm 0.4) \times 10^{21} \mathrm{~cm}^{-2}\right.$, photon index $\Gamma=$ $1.65 \pm 0.15$, and an amplitude of $(4.7 \pm 0.6) \times 10^{-4}$ photons $\mathrm{cm}^{-2} \mathrm{~s}^{-1} \mathrm{keV}^{-1}$ at $1 \mathrm{keV}]$. The residuals are plotted in the bottom panel.

per limit of $4 \mathrm{kpc}$ for SNR G160.9+2.6 and the full range of $0.8-1.6 \mathrm{kpc}$ for SNR G205.5+0.5 in Figure 30. Finally, SNR G156.2+5.7 is more highly absorbed than the other SNRs.

While all of the limits are below the luminosities of central sources in Cas A, Puppis A, and SNR G296.5+10.0 (and are therefore in concordance with our original survey design from Paper I), some are further below than others. The utility of these limits is somewhat lessened, however, as the SNRs are all reasonably large and are older (10-30 kyr) than the sources in Paper I (3-10 kyr). Therefore the cooling curves have descended, and there are other SNRs that have similar or even lower neutron star luminosities (CTA 1, IC 443, and W44 for the SNRs with tighter limits, and Vela and SNRs G114.3+0.3, G343.1-2.3, and G354.1+ 00.1 for the remaining SNRs), although $5 / 7$ of these sources have X-ray PWNe that increase their luminosities by a factor of $\sim 10$.

In one sense, however, the limits here are tighter than those of Paper I. By using the BSC to go to twice the SNR radii, we have virtually eliminated the possibility that there are high-velocity neutron stars in these SNRs (as discussed in $\S 3.1$, confusion is most likely not a limiting factor in detecting X-ray sources), while in Paper I we only searched a portion of the SNR interiors. It is of course possible that the supernova explosions were Type Ia or produced black holes, but as discussed in Paper I these alternate scenarios are not very likely for an ensemble.

Therefore, while not as tight as those of Paper I (or, e.g., Slane et al. 2002; Halpern et al. 2004), our limits are still useful. They are not below all detected neutron stars, so they do not require appeals to exotic physics or cooling processes, but they conclusively demonstrate that there is a significant range in the observed luminosities of neutron stars, even including experimental uncertainties. It is clear that the neutron stars of a single age must be able to produce luminosities differing by a factor of $>10$. Whether the unknown parameter that controls the luminosity is one of the usual culprits (mass, rotation, composition, magnetic field) or something entirely different is not known. It is also clear that there is a significant number of objects that do not show nonthermal emission and would therefore not go on to evolve as traditional radio pulsars. 

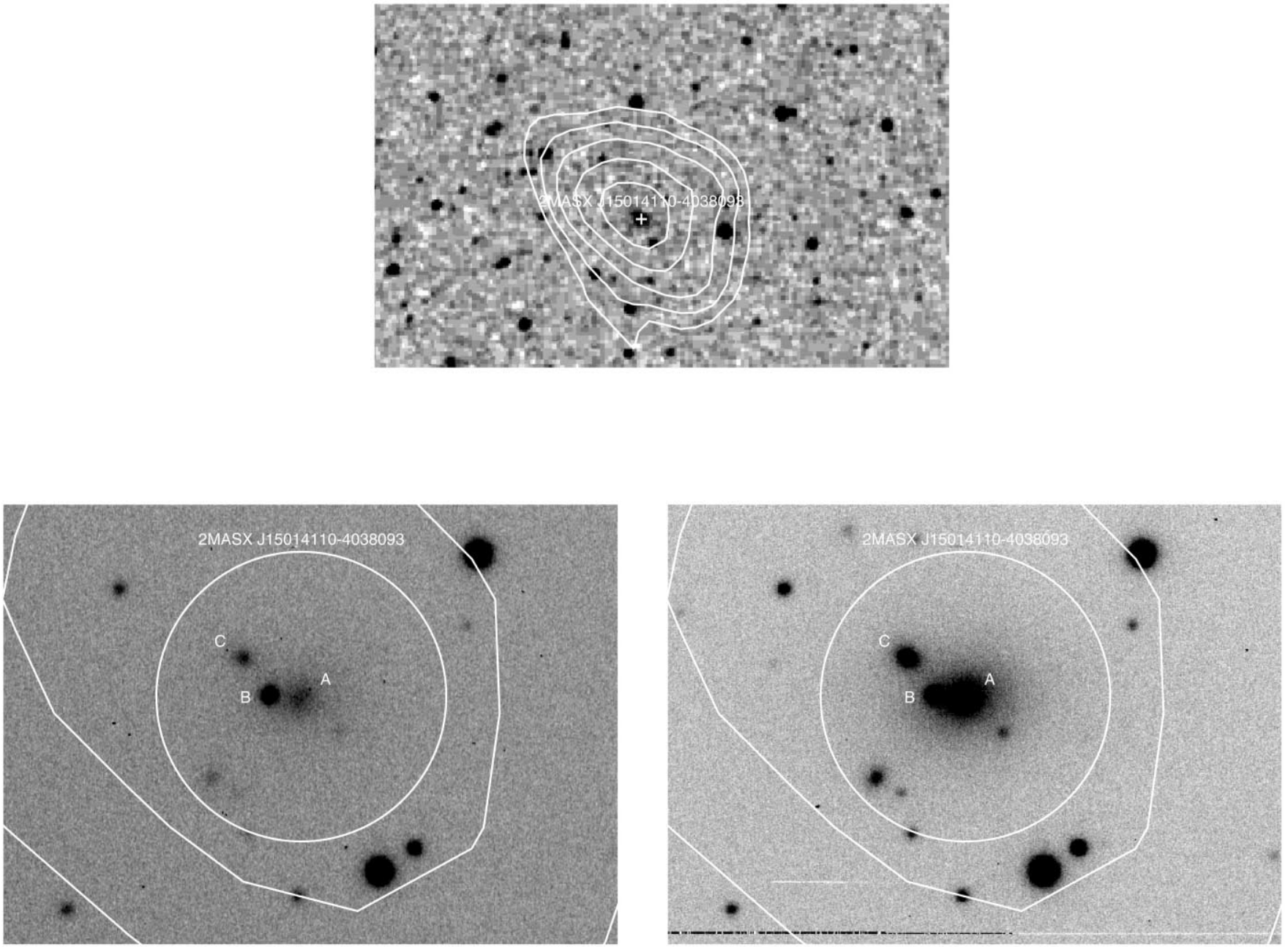

FIG. 29.-Top: 2MASS $K_{\mathrm{s}}$-band image of 1RXS J150139.6-403815. The image is $5^{\prime} \times 3.5$, with north up and east to the left. The contours from Fig. 27 are plotted, and the source 2MASX J15014110-4038093 is indicated by the plus sign. Bottom: Magellan $B$ (left) and $R$ (right) images of 1 RXS J150139.6-403815. The images are $40^{\prime \prime} \times 30^{\prime \prime}$, with north up and east to the left. Again, the contours from Fig. 27 are plotted, and the source 2MASX J15014110-4038093 is indicated by the circles $\left(10^{\prime \prime}\right.$ radius $)$.

TABLE 6

Properties of Optical Sources in Figure 29

\begin{tabular}{|c|c|c|c|c|c|c|}
\hline \multirow[b]{2}{*}{ Source } & \multirow[b]{2}{*}{$\begin{array}{c}\alpha-15^{\mathrm{h}} 01^{\mathrm{m}} \\
(\mathrm{s})\end{array}$} & \multirow[b]{2}{*}{$\begin{array}{c}-\delta-40^{\circ} 38^{\prime} \\
\quad(\operatorname{arcsec})\end{array}$} & \multicolumn{2}{|c|}{$B$} & \multicolumn{2}{|c|}{$R$} \\
\hline & & & $\begin{array}{l}\text { FWHM }^{\mathrm{a}} \\
(\operatorname{arcsec})\end{array}$ & Mag & $\begin{array}{l}\text { FWHM }^{\mathrm{b}} \\
(\operatorname{arcsec})\end{array}$ & Mag \\
\hline А & 41.12 & 09.4 & 3.2 & 12.1 & 3.6 & 9.5 \\
\hline В & 41.30 & 09.3 & 0.8 & 12.1 & 0.8 & 11.1 \\
\hline С & 41.46 & 06.7 & 1.3 & 12.9 & 3.6 & 11.2 \\
\hline
\end{tabular}

Notes.-Coordinates are J2000.0. The astrometry has absolute uncertainties of $\approx 0^{\prime \prime} 2$ in each coordinate owing to uncertainties in 2 MASS. The photometry has systematic uncertainties of $\approx 0.5 \mathrm{mag}$ owing to uncertain zero-point calibration.

a The seeing was $\approx 0$ ". 77 .

b The seeing was $\approx 0$ ". 66 . 


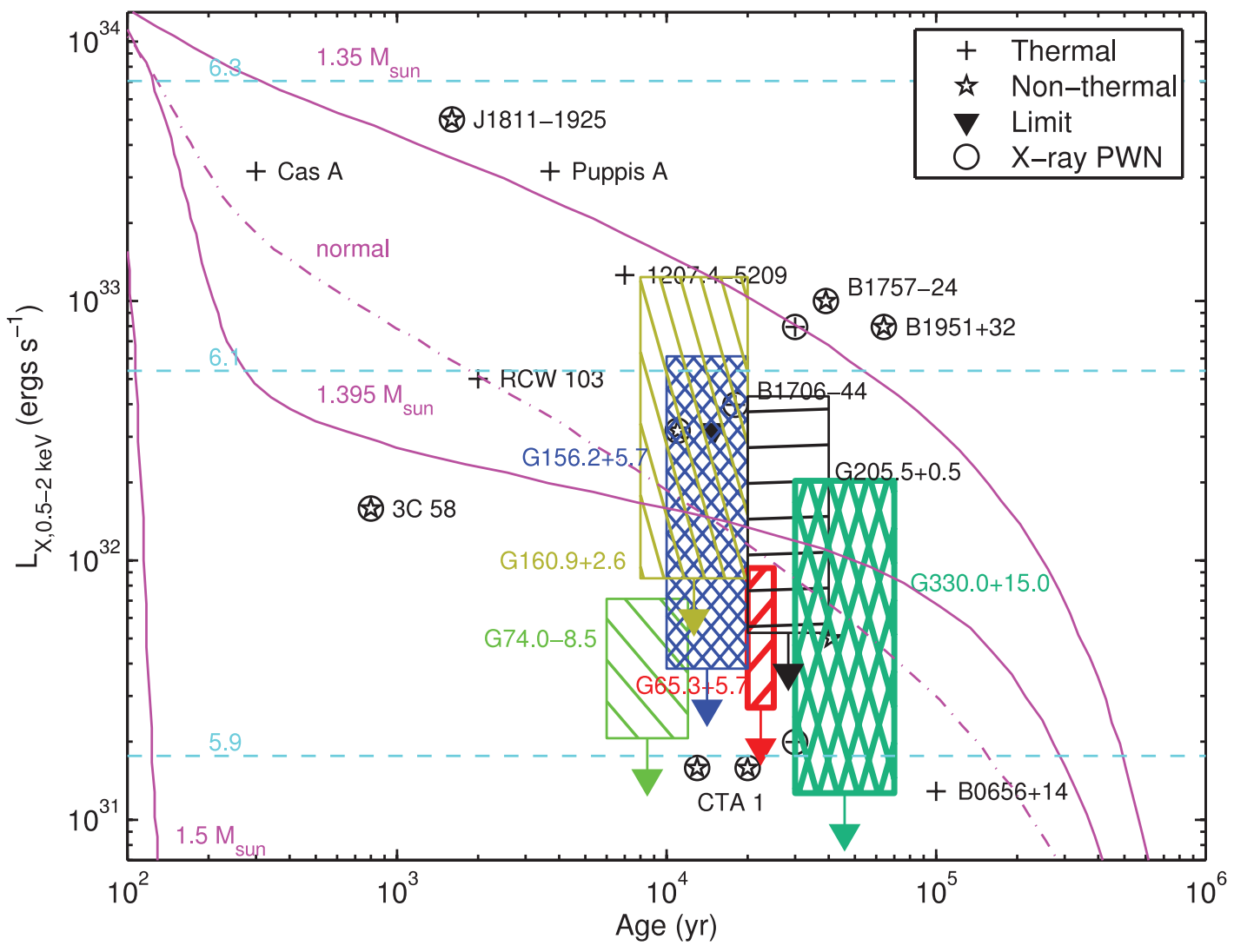

FIG. 30.-X-ray luminosities $(0.5-2 \mathrm{keV})$ as a function of age for young neutron stars. Sources whose emission is primarily thermal are indicated with plus signs, those whose emission is primarily nonthermal are indicated with stars, and those with only limits are indicated with triangles; see Paper I for source data and additional labels. The sources that have X-ray PWNe, which are typically $>10$ times the X-ray luminosity of the neutron stars themselves, are circled. We also plot the limits to blackbody emission from sources in SNRs G65.3+5.7 (red hatched region), G74.0-8.5 (green hatched region), G156.2+5.7 (blue cross-hatched region), G160.9+2.6 (gold hatched region), G205.5+0.5 (black hatched region), and G330.0+15.0 (dark green cross-hatched region). An uncertainty of 30\% (for SNRs with kinematic distances) or $60 \%$ (for SNRs with distances from X-ray fits) in the distance has been added to the luminosities given in Table 1 and $\S 2$, and the likely range of ages is also shown (for SNR G74.0-8.5, the luminosity has been increased to account for uncertain associations with 1RXS J205042.9+284643 and 1RXS J205812.8+292037). The cooling curves are the $1 p$ proton superfluid models from Yakovlev et al. (2004) (solid lines, with mass as labeled) and the normal (i.e., non-superfluid) $M=1.35 M_{\odot}$ model (dot-dashed line), assuming blackbody spectra and $R_{\infty}=10 \mathrm{~km}$. These curves are meant to be illustrative of general cooling trends and should not be interpreted as detailed predictions. The horizontal lines show the luminosity produced by blackbodies with $R_{\infty}=10 \mathrm{~km}$ and $\log T_{\infty}(\mathrm{K})$, as indicated. Compare to Fig. 37 of Paper I.

We thank an anonymous referee for helpful comments. D. L. K. was partially supported by a fellowship from the Fannie and John Hertz Foundation. B. M. G. and P. O. S. acknowledge support from NASA contract NAS8-39073 and grant G02-3090. B. M. G. is supported by NASA LTSA grant NAG5-13032. S. R. K. is supported by grants from NSF and NASA. Support for this work was provided by the National Aeronautics and Space Administration through Chandra award GO3-4088X issued by the Chandra X-Ray Observatory Center, which is operated by the Smithsonian Astrophysical Observatory for and on behalf of NASA under contract NAS8-39073. The NRAO is a facility of the National Science Foundation operated under cooperative agreement by Associated Universities, Inc. The Digitized Sky Surveys were produced at the Space Telescope Science Institute under US Government grant NAGW-2166. We have made extensive use of the SIMBAD database, and we are grateful to the astronomers at the Centre de Données Astronomiques de Strasbourg for maintaining this database. We would like to thank E. Persson and C. Rakowski for assistance with the PANIC observing, and D. Fox for assistance with the P60 observing.

Facilities: CXO (ACIS-S3), PO:1.5m (P60CCD), Magellan: Clay (MagIC,PANIC), Hale (LFC)
Arnaud, M., et al. 2002, A\&A, 390, 27

Baade, W., \& Zwicky, F. 1934, Proc. Natl. Acad. Sci., 20, 254

Bertin, E., \& Arnouts, S. 1996, A\&AS, 117, 393

Biggs, J. D., \& Lyne, A. G. 1996, MNRAS, 282, 691

Blair, W. P., Sankrit, R., Raymond, J. C., \& Long, K. S. 1999, AJ, 118, 942

Bock, D. C.-J., Large, M. I., \& Sadler, E. M. 1999, AJ, 117, 1578

Brown, B. A., \& Bregman, J. N. 1998, ApJ, 495, L75

Burrows, A., Ott, C. D., \& Meakin, C. 2004, in Cosmic Explosions in Three Dimensions, ed. P. Hoflich, P. Kumar, \& J. C. Wheeler (Cambridge: Cambridge Univ. Press), 209

Camilo, F. 2003, in ASP Conf. Ser. 302, Radio Pulsars, ed. M. Bailes, D. J.

Nice, \& S. E. Thorsett (San Fransisco: ASP), 145

Camilo, F., et al. 2002, ApJ, 571, L41

\section{REFERENCES}

Case, G. L., \& Bhattacharya, D. 1998, ApJ, 504, 761

Chatterjee, S., \& Cordes, J. M. 2002, ApJ, 575, 407

Chevalier, R. A. 2005, ApJ, 619, 839

Condon, J. J., Cotton, W. D., Greisen, E. W., Yin, Q. F., Perley, R. A., Taylor, G. B., \& Broderick, J. J. 1998, AJ, 115, 1693

Condon, J. J., \& Kaplan, D. L. 1998, ApJS, 117, 361

Cotter, G., Buttery, H. J., Das, R., Jones, M. E., Grainge, K., Pooley, G. G., \& Saunders, R. 2002, MNRAS, 334, 323

Damashek, M., Taylor, J. H., \& Hulse, R. A. 1978, ApJ, 225, L31

Drimmel, R., Cabrera-Lavers, A., \& López-Corredoira, M. 2003, A\&A, 409, 205

Fabbiano, G. 1989, ARA\&A, 27, 87

Fuhrmeister, B., \& Schmitt, J. H. M. M. 2003, A\&A, 403, 247 
Gaensler, B. M., \& Johnston, S. 1995, MNRAS, 275, L73

Gaensler, B. M., \& Slane, P. O. 2006, ARA\&A, in press (astro-ph/0601081)

Garmire, G. P., Bautz, M. W., Ford, P. G., Nousek, J. A., \& Ricker, G. R. 2003, Proc. SPIE, 4851, 28

Gorham, P. W., Ray, P. S., Anderson, S. B., Kulkarni, S. R., \& Prince, T. A. 1996, ApJ, 458, 257

Green, D. A. 2000, A Catalogue of Galactic Supernova Remnants (2000 August version; Cambridge: Mullard Radio Astronomy Observatory, Cavendish Laboratory), http://www.mrao.cam.ac.uk/surveys/snrs

Gull, T. R., Kirshner, R. P., \& Parker, R. A. R. 1977, ApJ, 215, L69

Halpern, J. P., Gotthelf, E. V., Camilo, F., Helfand, D. J., \& Ransom, S. M. 2004, ApJ, 612, 398

Hester, J. J., Graham, J. R., Beichman, C. A., \& Gautier, T. N. 1990, ApJ, 357, 539

Huang, Y.-L., \& Thaddeus, P. 1985, ApJ, 295, L13

Kaplan, D. L., Frail, D. A., Gaensler, B. M., Gotthelf, E. V., Kulkarni, S. R., Slane, P. O., \& Nechita, A. 2004, ApJS, 153, 269

Kaspi, V. M., \& Helfand, D. J. 2002, in ASP Conf. Ser. 271, Neutron Stars in Supernova Remnants, ed. P. O. Slane \& B. M. Gaensler (San Fransisco: ASP), 3

Kaspi, V. M., Manchester, R. N., Johnston, S., Lyne, A. G., \& D’Amico, N. 1996, AJ, 111, 2028

Kassim, N. E., Hertz, P., van Dyk, S. D., \& Weiler, K. W. 1994, ApJ, 427, L95

Kim, D., \& Fabbiano, G. 2004, ApJ, 611, 846

Kodama, T., \& Bower, R. 2003, MNRAS, 346, 1

Large, M. I., Vaughan, A. E., \& Mills, B. Y. 1968, Nature, 220, 340

Leahy, D. A., \& Aschenbach, B. 1995, A\&A, 293, 853

Leahy, D. A., Naranan, S., \& Singh, K. P. 1986, MNRAS, 220, 501

Leahy, D. A., Nousek, J., \& Hamilton, A. J. S. 1991, ApJ, 374, 218

Leahy, D. A., \& Roger, R. S. 1991, AJ, 101, 1033

Levenson, N. A., Graham, J. R., \& Walters, J. L. 2002, ApJ, 576, 798

Lorimer, D. R., Lyne, A. G., \& Camilo, F. 1998, A\&A, 331, 1002

Lozinskaya, T. A. 1981, Soviet Astron. Lett., 7, 17

Martini, P., Persson, S. E., Murphy, D. C., Birk, C., Shectman, S. A., Gunnels, S. M., \& Koch, E. 2004, Proc. SPIE, 5492, 1653

Matsushita, K., et al. 1994, ApJ, 436, L41

Mavromatakis, F., Boumis, P., Papamastorakis, J., \& Ventura, J. 2002, A\&A, 388,355

Miyata, E., Ohta, K., Torii, K., Takeshima, T., Tsunemi, H., Hasegawa, T., \& Hashimoto, Y. 2001, ApJ, 550, 1023

Miyata, E., Tsunemi, H., Kohmura, T., Suzuki, S., \& Kumagai, S. 1998a, PASJ, 50,257
Miyata, E., et al. 1998b, PASJ, 50, 475

Mulchaey, J. S., Davis, D. S., Mushotzky, R. F., \& Burstein, D. 2003, ApJS, 145,39

Mushotzky, R. F. 2004, in Clusters of Galaxies: Probes of Cosmological Structure and Galaxy Evolution, ed. J. S. Mulchaey, A. Dressler, \& A. Oemler (Cambridge: Cambridge Univ. Press), 123

Odegard, N. 1986, ApJ, 301, 813

Pfeffermann, E., Aschenbach, B., \& Predehl, P. 1991, A\&A, 246, L28

Predehl, P., \& Schmitt, J. H. M. M. 1995, A\&A, 293, 889

Raymond, J. C., \& Smith, B. W. 1977, ApJS, 35, 419

Richman, H. R. 1996, ApJ, 462, 404

Rutledge, R. E., Fox, D. W., Bogosavljevic, M., \& Mahabal, A. 2003, ApJ, 598,458

Schaudel, D., Becker, W., Lu, F., \& Aschenbach, B. 2002, in Abstracts from 34th COSPAR Scientific Assembly (Paris: COSPAR)

Seward, F. D., Gorenstein, P., \& Smith, R. K. 2006, ApJ, 636, 873

Shelton, R. L., Kuntz, K. D., \& Petre, R. 2004, ApJ, 615, 275

Skrutskie, M. F., et al. 1997, in The Impact of Large Scale Near-IR Sky Surveys, F. Garzon et al. (Dordrecht: Kluwer), 25

Slane, P. O., Helfand, D. J., \& Murray, S. S. 2002, ApJ, 571, L45

Staelin, D. H., \& Reifenstein, E. C. 1968, Science, 162, 1481

Stappers, B. W., Gaensler, B. M., Kaspi, V. M., van der Klis, M., \& Lewin, W. H. G. 2003, Science, 299, 1372

Stelzer, B., Huélamo, N., Hubrig, S., Zinnecker, H., \& Micela, G. 2003, A\&A, 407, 1067

Tananbaum, H. 1999, IAU Circ., 7246, 1

Ueda, Y., Ishisaki, Y., Takahashi, T., Makishima, K., \& Ohashi, T. 2001, ApJS, 133,1

van der Swaluw, E., Downes, T. P., \& Keegan, R. 2004, A\&A, 420, 937

Verbunt, F., Bunk, W. H., Ritter, H., \& Pfeffermann, E. 1997, A\&A, 327, 602

Vikhlinin, A., Forman, W., \& Jones, C. 1999a, ApJ, 525, 47

Vikhlinin, A., McNamara, B. R., Hornstrup, A., Quintana, H., Forman, W., Jones, C., \& Way, M. 1999b, ApJ, 520, L1

Voges, W., et al. 1999, A\&A, 349, 389

White, D. A., Jones, C., \& Forman, W. 1997, MNRAS, 292, 419

Yakovlev, D. G., Gnedin, O. Y., Kaminker, A. D., Levenfish, K. P., \& Potekhin, A. Y. 2004, Adv. Space Res., 33, 523

Yamauchi, S., Koyama, K., Tomida, H., Yokogawa, J., \& Tamura, K. 1999 PASJ, 51, 13 